\title{
ERROR ESTIMATES FOR THE NUMERICAL APPROXIMATION OF A DISTRIBUTED CONTROL PROBLEM FOR THE STEADY-STATE NAVIER-STOKES EQUATIONS*
}

\author{
EDUARDO CASAS ${ }^{\dagger}$, MARIANO MATEOS $\ddagger$, AND JEAN-PIERRE RAYMOND $§$
}

\begin{abstract}
We obtain error estimates for the numerical approximation of a distributed control problem governed by the stationary Navier-Stokes equations, with pointwise control constraints. We show that the $L^{2}$-norm of the error for the control is of order $h^{2}$ if the control set is not discretized, while it is of order $h$ if it is discretized by piecewise constant functions. These error estimates are obtained for local solutions of the control problem, which are nonsingular in the sense that the linearized Navier-Stokes equations around these solutions define some isomorphisms, and which satisfy a second order sufficient optimality condition. We establish a second order necessary optimality condition. The gap between the necessary and sufficient second order optimality conditions is the usual gap known for finite dimensional optimization problems.
\end{abstract}

Key words. optimal control, stationary Navier-Stokes equations, numerical approximation, error estimates

AMS subject classifications. 65N30, 65N15, 49M05, 49M25

DOI. $10.1137 / 060649999$

1. Introduction. The goal of this paper is to derive some error estimates for the numerical approximation of a distributed optimal control problem governed by the steady-state Navier-Stokes equations, with pointwise control constraints. More precisely we consider the following problem:

$$
\inf \left\{F(\mathbf{u}, \mathbf{y}) \mid \mathbf{u} \in U_{a d} \text { and }(\mathbf{u}, \mathbf{y}) \text { satisfies }(1.2)\right\},
$$

where

$$
\begin{gathered}
F(\mathbf{u}, \mathbf{y})=\frac{1}{2} \int_{\Omega}\left|\mathbf{y}(x)-\mathbf{y}_{d}(x)\right|^{2} d x+\frac{N}{2} \int_{\omega}|\mathbf{u}(x)|^{2} d x, \\
-\nu \Delta \mathbf{y}+(\mathbf{y} \cdot \nabla) \mathbf{y}+\nabla p=\mathbf{f}+\mathcal{C} \mathbf{u} \text { in } \Omega, \quad \operatorname{div} \mathbf{y}=0 \text { in } \Omega, \quad \mathbf{y}=0 \text { on } \Gamma,
\end{gathered}
$$

$\mathcal{C}$ is a localization operator, $\omega \subset \Omega, N>0, \nu>0$, and

$$
U_{a d}=\left\{\mathbf{u} \in L^{2}\left(\omega ; \mathbb{R}^{m}\right) \mid \alpha \leq \mathbf{u}(x) \leq \beta \text { for almost every (a.e.) } x \in \omega\right\} .
$$

In this setting, $\Omega$ is a bounded open and connected subset in $\mathbb{R}^{d}$, of class $C^{2}$, with $d=2$ or $d=3$, and $\omega$ is a nonempty open subset in $\Omega$. We can easily show that

\footnotetext{
* Received by the editors January 14, 2006; accepted for publication (in revised form) January 9, 2007; published electronically June 28, 2007. The first two authors were partially supported by the Spanish Ministry of Education and Science under projects MTM2005-06817 and "Ingenio Mathematica (i-MATH)" CSD2006-00032 (Consolider Ingenio 2010).

http://www.siam.org/journals/sicon/46-3/64999.html

${ }^{\dagger}$ Departmento de Matemática Aplicada y Ciencias de la Computación, E.T.S.I. Industriales y de Telecomunicación, Universidad de Cantabria, 39005 Santander, Spain (eduardo.casas@unican.es).

${ }^{\ddagger}$ Departmento de Matemáticas, E.P. de Gijón, Universidad de Oviedo, Campus de Viesques, 33203 Gijón, Spain (mmateos@uniovi.es).

§Laboratoire MIP, UMR CNRS 5640, Université Paul Sabatier, 31062 Toulouse Cedex 9, France (raymond@mip.ups-tlse.fr).
} 
problem (P) admits at least one solution. On one hand, uniqueness of solution to problem (P) is not necessarily guaranteed even if (1.2) has a unique solution (which is not necessarily the case). On the other hand, we can only hope to obtain error estimates for solutions to problem (P) which are locally unique. Local uniqueness can be proved for solutions satisfying first order and sufficient second order optimality conditions. When first order optimality conditions in qualified form are satisfied by a local solution $(\overline{\mathbf{u}}, \overline{\mathbf{y}})$ of problem $(\mathrm{P})$, we have

$$
\overline{\mathbf{u}}=\operatorname{Proj}_{[\alpha, \beta]}\left(-\frac{1}{N} \mathcal{C}^{*} \overline{\mathbf{\Phi}}\right),
$$

where $\operatorname{Proj}_{[\alpha, \beta]}$ is a projection operator and $\overline{\mathbf{\Phi}}$ is the adjoint state associated with $(\overline{\mathbf{u}}, \overline{\mathbf{y}})$. Thus, even if $\overline{\mathbf{\Phi}}$ is regular, because of the projection operator $\operatorname{Proj}_{[\alpha, \beta]}$ (due to control constraints), $\overline{\mathbf{u}}$ is only a Lipschitz function.

Assuming that $(\overline{\mathbf{u}}, \overline{\mathbf{y}})$ satisfies first order and sufficient second order optimality conditions, we can define a discrete control problem $\left(\mathrm{P}_{h}\right)$ by discretizing the state equation (1.2) with a finite element method (here $h$ is the mesh size of the underlying triangulation, and we assume that the family of triangulations is regular; see section 4). We consider two cases, the case where the control set in $\left(\mathrm{P}_{h}\right)$ is still $U_{a d}$, and the case where the control set $U_{a d}^{h}$ is the set of functions in $U_{a d}$ which are piecewise constant on the elements of the triangulation. We show that there exists $\hat{h}$ such that, for all $0<h \leq \hat{h}$, the discrete control problem $\left(\mathrm{P}_{h}\right)$ admits at least one local solution $\overline{\mathbf{u}}_{h}$ in a ball $B_{\rho}(\overline{\mathbf{u}})$. We prove that the corresponding sequences $\left\{\overline{\mathbf{u}}_{h}\right\}_{h}$ strongly converge to $\overline{\mathbf{u}}$ in $L^{2}$ (see Theorem 4.11). When the control set in $\left(\mathrm{P}_{h}\right)$ is $U_{a d}$, we show that

$$
\left\|\overline{\mathbf{u}}_{h}-\overline{\mathbf{u}}\right\|_{L^{2}} \leq C h^{2}
$$

while if the control set is $U_{a d}^{h}$, we prove that

$$
\left\|\overline{\mathbf{u}}_{h}-\overline{\mathbf{u}}\right\|_{L^{2}} \leq C h
$$

(see Theorem 4.18). To the best of our knowledge both results are new. For numerical computations it seems easier to solve $\left(\mathrm{P}_{\mathrm{h}}\right)$ when the control set is discretized, that is, when controls belong to $U_{a d}^{h}$. However, it is also possible to solve it without a priori discretizing the control set (see, e.g., [16]).

Before comparing our results with the ones existing in the literature, let us make some comments. Knowing that $\overline{\mathbf{u}}$ is a Lipschitz function, the error estimate (1.4), obtained when the discrete control set is defined with piecewise constant functions, is consistent with estimates obtained by approximating Lipschitz functions by piecewise constant functions. The result obtained in (1.3) is more surprising. Indeed, as we are going to see, this kind of result is already known for problems without control constraints. But in that case the optimal control belongs to $H^{2}$, and the error estimate is then directly derived from error estimates for the adjoint state. Here we obtain the same order of error estimate, but with control constraints. As far as we know, this kind of result was not previously known. Moreover, our method is quite general, and it can be used in some other problems, provided that we are able to obtain error estimates for the discrete state and discrete adjoint equations.

Let us come back to the existing results in the literature. For optimal control problems of the steady-state Navier-Stokes equations with a distributed control and a slightly different functional, Gunzburger, Hou, and Svobodny have proved error 
estimates similar to (1.3) in the case when there is no control constraints and when the control acts everywhere in $\Omega$ (see [13, end of section 5.2]). But for a distributed control localized in $\Omega$, the error estimate is only of order $h^{3 / 2-\varepsilon}$ (see [13, end of section 5.3]). To prove these estimates they do not assume that the optimal solution $(\overline{\mathbf{u}}, \overline{\mathbf{y}})$, which they want to approximate, satisfies a sufficient second order optimality condition. But they assume that the optimality system satisfied by $(\overline{\mathbf{u}}, \overline{\mathbf{y}})$ is regular, in the sense that the corresponding linearized optimality system defines some isomorphism. This approach is the extension - to optimality systems of control problems - of the classical one used in the numerical approximation of the steady-state Navier-Stokes equations; see, e.g., [12]. This method has been used in the literature for other similar problems [17] and for the boundary control of the stationary Navier-Stokes equations $[14,15]$. Observe that the estimates are not the same if the boundary of the domain where the control is applied is empty or nonempty [14, Theorem 4.6 and the assumptions in Theorem 3.5]. In any case this method cannot be used for problems with control constraints. Another approach used more recently for problems without control constraints is the one by Deckelnick and Hinze [10], which is based on the Kantorovich convergence theorem of the Newton method. In that case a second order sufficient optimality condition is needed, but the Kantorovich convergence theorem is proved only for systems of equations and not for generalized equations. Thus this method cannot be used for problems with control constraints.

For problems with control constraints the obtention of both optimality conditions and error estimates is more complicated. Indeed even if the nonlinear Navier-Stokes equations are well posed, the linearized ones are not necessarily well posed. Thus in general one can obtain optimality conditions only in nonqualified form, that is, optimality conditions of Fritz-John type. Such optimality conditions for optimal control problems of the stationary Navier-Stokes equations have been obtained by Abergel and Casas [1]; see also Casas [3]. Optimality conditions in qualified form, that is, optimality conditions of Karush-Kuhn-Tucker type, may be obtained either by assuming that data of the problem are small enough with respect to the viscosity parameter $\nu$ (see, e.g., Roubiček and Tröltzsch [19], Tröltzsch and Wachsmuth [21], De Los Reyes [18]) or by assuming some qualification condition of the set of feasible controls as in Gunzburger, Hou, and Svobodny [15, condition (2.7)] or in [1].

Here, since we are mainly interested in the numerical approximation of control problem $(\mathrm{P})$, we assume that the local optimal solution $(\overline{\mathbf{u}}, \overline{\mathbf{y}})$ we want to approximate is a nonsingular solution, that is, that the linearized Navier-Stokes equations about $\overline{\mathbf{y}}$ define some isomorphism. As already mentioned, this is the classical assumption used in the numerical approximation of the Navier-Stokes equations (see, e.g., [12, p. 297]). Thanks to this assumption we derive a necessary optimality condition of the form

$$
J^{\prime \prime}(\overline{\mathbf{u}}) \mathbf{v}^{2} \geq 0 \quad \forall \mathbf{v} \in C_{\overline{\mathbf{u}}},
$$

where $C_{\overline{\mathbf{u}}}$ is the set of directions belonging to the tangent cone at $\overline{\mathbf{u}}$ to $U_{a d}$ satisfying $J^{\prime}(\overline{\mathbf{u}}) \mathbf{v}=0$; see Theorem 3.6 and Corollary 3.7 (here $J(\mathbf{u})=F\left(\mathbf{u}, \mathbf{y}_{\mathbf{u}}\right)$, where $\mathbf{y}_{\mathbf{u}}$ is the unique solution to (1.2) corresponding to $\mathbf{u}$, when $\mathbf{u}$ belongs to some ball $\left.B_{\rho}(\overline{\mathbf{u}})\right)$. The weakest sufficient optimality condition we can state is the following:

$$
J^{\prime \prime}(\overline{\mathbf{u}}) \mathbf{v}^{2}>0 \quad \forall \mathbf{v} \in C_{\overline{\mathbf{u}}} \text { such that } \mathbf{v} \neq 0 .
$$

Under this condition, and assuming that the first order optimality conditions are in qualified form, we prove that $(\overline{\mathbf{u}}, \overline{\mathbf{y}})$ is the unique local solution to $(\mathrm{P})$ in some ball 
$B_{\rho}(\overline{\mathbf{u}})$. (See Theorem 3.8. Notice that we cannot hope to prove such a result without assuming that $\overline{\mathbf{u}}$ satisfies the first order optimality conditions in qualified form and condition (1.6).) This local uniqueness result is essential to carry out some numerical analysis of the control problem. The discrete state equation is stated in section 4 . The well posedness of the discrete state equation is performed in Theorem 4.8, and error estimates are obtained in Lemma 4.10. The discrete adjoint equation is studied in section 4.3. Its well posedness and error estimates are proved in Lemmas 4.12 and 4.13. Error estimates for the control problem are obtained in section 4.4.

Let us finally mention that in the case of control problems governed by scalar semilinear elliptic equations, this approach to derive error estimates has been developed by Arada, Casas, and Tröltzsch [2], Casas [4, 5], Casas, Mateos, and Tröltzsch [6], and Casas and Raymond [7].

2. Assumptions and preliminary results. Let us recall that $\Omega$ is a bounded open and connected subset in $\mathbb{R}^{d}$, of class $C^{2}$, with $d=2$ or $d=3$, and that $\omega$ is a nonempty open subset in $\Omega$. We assume that $M: \omega \rightarrow \mathbb{R}^{d \times m}$ is a Lipschitz function, with $1 \leq m \leq d$ ( $\mathbb{R}^{d \times m}$ denotes the space of $d \times m$ real matrices). Let us consider the linear operator $\mathcal{C} \in \mathcal{L}\left(L^{2}\left(\omega ; \mathbb{R}^{m}\right), L^{2}\left(\Omega ; \mathbb{R}^{d}\right)\right)$, defined by $(\mathcal{C u})(x)=M(x) \mathbf{u}(x) \chi_{\omega}(x)$, where $\chi_{\omega}$ is the characteristic function of $\omega$. In the functional $F: L^{2}\left(\Omega ; \mathbb{R}^{d}\right) \times$ $L^{2}\left(\omega ; \mathbb{R}^{m}\right) \longmapsto \mathbb{R}$, defined in (1.1), we assume that $N>0$ and $\mathbf{y}_{d} \in L^{\bar{r}}\left(\Omega ; \mathbb{R}^{d}\right)$, for some $\bar{r}>d$, are given fixed. For $\mathbf{u} \in L^{2}\left(\omega ; \mathbb{R}^{m}\right)$, we denote by $u_{j}$ the components of $\mathbf{u}$, that is, $\mathbf{u}=\left(u_{j}\right)_{1 \leq j \leq m}$. For $1 \leq j \leq m$, let $-\infty \leq \alpha_{j}<\beta_{j} \leq+\infty$ be extended real numbers, and set

$$
U_{a d}=\left\{\mathbf{u} \in L^{2}\left(\omega ; \mathbb{R}^{m}\right) \mid \alpha_{j} \leq u_{j}(x) \leq \beta_{j} \text { for a.e. } x \in \omega, 1 \leq j \leq m\right\} .
$$

In the case when $\alpha_{j}=-\infty$, this means that the corresponding constraint is absent. The same convention is adopted if $\beta_{j}=\infty$.

In (1.2) we assume that $\nu>0$ and $\mathbf{f} \in L^{\bar{r}}\left(\Omega ; \mathbb{R}^{d}\right)$.

To study (1.2) we have to introduce some function spaces and operators. Throughout the following we set $\mathbf{H}^{1}(\Omega)=H^{1}\left(\Omega ; \mathbb{R}^{d}\right), \mathbf{H}_{0}^{1}(\Omega)=H_{0}^{1}\left(\Omega ; \mathbb{R}^{d}\right), \mathbf{H}^{-1}(\Omega)=$ $\left(\mathbf{H}_{0}^{1}(\Omega)\right)^{\prime}, \mathbf{L}^{p}(\Omega)=L^{p}\left(\Omega ; \mathbb{R}^{d}\right)$, and $\mathbf{W}^{s, p}(\Omega)=W^{s, p}\left(\Omega ; \mathbb{R}^{d}\right)$ for $1 \leq p \leq \infty$ and $s>0$. We introduce different spaces of divergence-free vector fields:

$$
\begin{aligned}
& \mathbf{V}_{n}^{0}(\Omega)=\left\{\mathbf{u} \in \mathbf{L}^{2}(\Omega) \mid \operatorname{div} \mathbf{u}=0 \text { in } \Omega, \mathbf{u} \cdot \mathbf{n}=0 \text { in } H^{-1 / 2}(\Gamma)\right\}, \\
& \mathbf{V}_{0}^{1}(\Omega)=\mathbf{H}_{0}^{1}(\Omega) \cap \mathbf{V}_{n}^{0}(\Omega),
\end{aligned}
$$

where $\mathbf{n}$ is the outward unit normal to $\Gamma$. The dual space of $\mathbf{V}_{0}^{1}(\Omega)$ with respect to the pivot space $\mathbf{V}_{n}^{0}(\Omega)$ is denoted by $\mathbf{V}^{-1}(\Omega)$. Thus we have

$$
\mathbf{V}_{0}^{1}(\Omega) \hookrightarrow \mathbf{V}_{n}^{0}(\Omega) \hookrightarrow \mathbf{V}^{-1}(\Omega),
$$

with dense and continuous imbeddings. The orthogonal projector from $\mathbf{L}^{2}(\Omega)$ onto $\mathbf{V}_{n}^{0}(\Omega)$ will be denoted by $P$. The operator $P$ can be extended to a bounded operator from $\mathbf{H}^{-1}(\Omega)$ to $\mathbf{V}^{-1}(\Omega)$. For notational simplicity this extension will still be denoted by $P$.

Let us consider the bilinear form on $\mathbf{H}_{0}^{1}(\Omega)$ defined by

$$
a(\mathbf{y}, \mathbf{z})=\nu \int_{\Omega} \nabla \mathbf{y}: \nabla \mathbf{z} d x=\nu \sum_{i, j=1}^{d} \int_{\Omega} \partial_{x_{i}} y_{j} \partial_{x_{i}} z_{j},
$$


and the trilinear form on $b: \mathbf{L}^{4}(\Omega) \times \mathbf{H}_{0}^{1}(\Omega) \times \mathbf{L}^{4}(\Omega)$ defined by

$$
b(\mathbf{y}, \mathbf{z}, \mathbf{\Phi})=\int_{\Omega}(\mathbf{y} \cdot \nabla) \mathbf{z} \cdot \mathbf{\Phi} d x
$$

We define $A \in \mathcal{L}\left(\mathbf{H}_{0}^{1}(\Omega), \mathbf{H}^{-1}(\Omega)\right)$ by

$$
\langle A \mathbf{y}, \mathbf{z}\rangle_{\mathbf{H}^{-1}(\Omega), \mathbf{H}_{0}^{1}(\Omega)}=a(\mathbf{y}, \mathbf{z}) \quad \forall \mathbf{z}, \mathbf{y} \in \mathbf{H}_{0}^{1}(\Omega),
$$

and the nonlinear operator $B$ from $\mathbf{H}_{0}^{1}(\Omega)$ to $\mathbf{H}^{-1}(\Omega)$ by

$$
\langle B(\mathbf{y}), \mathbf{z}\rangle_{\mathbf{H}^{-1}(\Omega), \mathbf{H}_{0}^{1}(\Omega)}=b(\mathbf{y}, \mathbf{y}, \mathbf{z}) \quad \forall \mathbf{z}, \mathbf{y} \in \mathbf{H}_{0}^{1}(\Omega) .
$$

Equation (1.2) is equivalent to the variational problem

$$
\begin{aligned}
& \text { Find } \mathbf{y} \in \mathbf{V}_{0}^{1}(\Omega) \quad \text { such that } \\
& a(\mathbf{y}, \mathbf{z})+b(\mathbf{y}, \mathbf{y}, \mathbf{z})=(\mathbf{f}+\mathcal{C} \mathbf{u}, \mathbf{z}) \quad \forall \mathbf{z} \in \mathbf{V}_{0}^{1}(\Omega),
\end{aligned}
$$

or to the weak formulation

$$
\mathbf{y} \in \mathbf{V}_{0}^{1}(\Omega), \quad\langle A \mathbf{y}+B(\mathbf{y}), \mathbf{z}\rangle_{\mathbf{H}^{-1}(\Omega), \mathbf{H}_{0}^{1}(\Omega)}=\langle\mathbf{f}+\mathcal{C} \mathbf{u}, \mathbf{z}\rangle_{\mathbf{H}^{-1}(\Omega), \mathbf{H}_{0}^{1}(\Omega)} \quad \forall \mathbf{z} \in \mathbf{V}_{0}^{1}(\Omega) .
$$

This last equation is equivalent to

$$
\mathbf{y} \in \mathbf{V}_{0}^{1}(\Omega), \quad P A \mathbf{y}+P B(\mathbf{y})=P(\mathbf{f}+\mathcal{C} \mathbf{u}) \quad \text { in } \mathbf{V}^{-1}(\Omega),
$$

which we shall simply write in the form

$$
\mathbf{y} \in \mathbf{V}_{0}^{1}(\Omega), \quad A \mathbf{y}+B(\mathbf{y})=\mathbf{f}+\mathcal{C} \mathbf{u} \quad \text { in } \mathbf{V}^{-1}(\Omega) .
$$

We know that, for all $\mathbf{u} \in L^{2}\left(\omega ; \mathbb{R}^{m}\right)$, equation (2.1), or equivalently (2.2), admits at least one solution $\mathbf{y} \in \mathbf{V}_{0}^{1}(\Omega)$. The pressure appearing in (1.2) is the unique function in

$$
L_{0}^{2}(\Omega)=\left\{v \in L^{2}(\Omega): \int_{\Omega} v(x) d x=0\right\}
$$

obeying

$$
\nabla p=(I-P)(\mathbf{f}+\mathcal{C} \mathbf{u}+\nu \Delta \mathbf{y}-(\mathbf{y} \cdot \nabla) \mathbf{y}) .
$$

It is a consequence of [12, Chapter 1, Lemma 2.1].

The following properties are well known. For all $\mathbf{y} \in \mathbf{L}^{4}(\Omega)$ obeying $\operatorname{div} \mathbf{y}=0$ in $\Omega$, and $\mathbf{z}, \mathbf{w} \in \mathbf{H}_{0}^{1}(\Omega)$

$$
b(\mathbf{y}, \mathbf{z}, \mathbf{w})=-b(\mathbf{y}, \mathbf{w}, \mathbf{z}) \quad \text { and } \quad b(\mathbf{y}, \mathbf{z}, \mathbf{z})=0 .
$$

The next lemma follows directly from Green's formula.

Lemma 2.1. For all $\mathbf{y} \in \mathbf{H}_{0}^{1}(\Omega)$, the operators $B^{\prime}(\mathbf{y}) \in \mathcal{L}\left(\mathbf{H}_{0}^{1}(\Omega), \mathbf{H}^{-1}(\Omega)\right)$ and $B^{\prime}(\mathbf{y})^{*} \in \mathcal{L}\left(\mathbf{H}_{0}^{1}(\Omega), \mathbf{H}^{-1}(\Omega)\right)$ satisfy

$$
\left\langle B^{\prime}(\mathbf{y}) \mathbf{z}, \mathbf{\Phi}\right\rangle=b(\mathbf{y}, \mathbf{z}, \mathbf{\Phi})+b(\mathbf{z}, \mathbf{y}, \mathbf{\Phi})
$$


and

$$
\left\langle B^{\prime}(\mathbf{y})^{*} \mathbf{\Phi}, \mathbf{z}\right\rangle=\int_{\Omega}(\nabla \mathbf{y})^{T} \mathbf{\Phi} \cdot \mathbf{z} d x-b(\mathbf{y}, \mathbf{\Phi}, \mathbf{z})-\int_{\Omega}(\operatorname{div} \mathbf{y}) \mathbf{\Phi} \cdot \mathbf{z} d x
$$

for all $\mathbf{z}, \boldsymbol{\Phi} \in \mathbf{H}_{0}^{1}(\Omega)$. Moreover, $B^{\prime \prime} \in \mathcal{L}\left(\mathbf{H}_{0}^{1}(\Omega) \times \mathbf{H}_{0}^{1}(\Omega), \mathbf{H}^{-1}(\Omega)\right)$ obeys

$$
\left\langle B^{\prime \prime}(\mathbf{y}, \mathbf{z}), \boldsymbol{\Phi}\right\rangle=b(\mathbf{y}, \mathbf{z}, \boldsymbol{\Phi})+b(\mathbf{z}, \mathbf{y}, \mathbf{\Phi}) \quad \forall \mathbf{z}, \mathbf{y}, \mathbf{\Phi} \in \mathbf{H}_{0}^{1}(\Omega)
$$

The following regularity result will be used throughout this paper. It is an immediate consequence of the classical result by Cattabriga [8].

THEOREM 2.2. There exists a constant $C>0$ such that if $\mathbf{u} \in L^{2}\left(\omega ; \mathbb{R}^{m}\right)$ and if $\mathbf{y} \in \mathbf{V}_{0}^{1}(\Omega)$ is a solution to (2.2), then

$$
\|\mathbf{y}\|_{\mathbf{V}_{0}^{1}(\Omega)} \leq C\left(\|\mathbf{f}\|_{\mathbf{L}^{2}(\Omega)}+\|\mathbf{u}\|_{L^{2}\left(\omega ; \mathbb{R}^{m}\right)}\right) .
$$

There exists a constant $C_{r}>0$ such that if $\mathbf{u} \in L^{r}\left(\omega ; \mathbb{R}^{m}\right), \mathbf{f} \in \mathbf{L}^{r}(\Omega)$ with $2 \leq r<\infty$ and $\mathbf{y} \in \mathbf{V}_{0}^{1}(\Omega)$ is a solution to (2.2) and $p$ the associated pressure, then $\mathbf{y} \in \mathbf{W}^{2, r}(\Omega)$, $p \in \mathbf{W}^{1, r}(\Omega)$, and

$$
\|p\|_{\mathbf{W}^{1, r}(\Omega)}+\|\mathbf{y}\|_{\mathbf{W}^{2, r}(\Omega)} \leq C_{r}\left(1+\|\mathbf{f}\|_{\mathbf{L}^{r}(\Omega)}^{7}+\|\mathbf{u}\|_{L^{r}\left(\omega ; \mathbb{R}^{m}\right)}^{7}\right) .
$$

Proof. The estimate of $\|\mathbf{y}\|_{\mathbf{V}_{0}^{1}(\Omega)}$ is classical. Using this estimate, since $d \leq 3$, we can write

$$
\|\mathbf{y}\|_{\mathbf{L}^{6}(\Omega)} \leq C\|\mathbf{y}\|_{\mathbf{V}_{0}^{1}(\Omega)}
$$

and

$$
\|\mathbf{y} \otimes \mathbf{y}\|_{\left(\mathbf{L}^{3}(\Omega)\right)^{d}} \leq C\|\mathbf{y}\|_{\mathbf{V}_{0}^{1}(\Omega)}^{2} .
$$

Thus, from estimates for the Stokes equation, we successively deduce

$$
\begin{aligned}
\|\mathbf{y}\|_{\mathbf{W}^{1,3}(\Omega)} \leq & C\left(\|\mathbf{y} \otimes \mathbf{y}\|_{\left(\mathbf{L}^{3}(\Omega)\right)^{d}}+\|\mathbf{f}\|_{\mathbf{L}^{2}(\Omega)}+\|\mathbf{u}\|_{L^{2}\left(\omega ; \mathbb{R}^{m}\right)}\right) \\
& \leq C\left(\|\mathbf{f}\|_{\mathbf{L}^{2}(\Omega)}^{2}+\|\mathbf{u}\|_{L^{2}\left(\omega ; \mathbb{R}^{m}\right)}^{2}+1\right)
\end{aligned}
$$

and

$$
\begin{gathered}
\|\mathbf{y}\|_{\mathbf{H}^{2}(\Omega)} \leq C\left(\|\mathbf{y}\|_{\mathbf{W}^{1,3}(\Omega)}\|\mathbf{y}\|_{\mathbf{L}^{6}(\Omega)}+\|\mathbf{f}\|_{\mathbf{L}^{2}(\Omega)}+\|\mathbf{u}\|_{L^{2}\left(\omega ; \mathbb{R}^{m}\right)}\right) \\
\leq C\left(\|\mathbf{f}\|_{\mathbf{L}^{2}(\Omega)}^{3}+\|\mathbf{u}\|_{L^{2}\left(\omega ; \mathbb{R}^{m}\right)}^{3}+1\right)
\end{gathered}
$$

Therefore

$$
\|(\mathbf{y} \cdot \nabla) \mathbf{y}\|_{\left(\mathbf{L}^{3}(\Omega)\right)^{d}} \leq C\|\mathbf{y}\|_{\mathbf{H}^{1}(\Omega)}\|\mathbf{y}\|_{\mathbf{H}^{2}(\Omega)} \leq C\left(\|\mathbf{f}\|_{\mathbf{L}^{2}(\Omega)}^{4}+\|\mathbf{u}\|_{L^{2}\left(\omega ; \mathbb{R}^{m}\right)}^{4}+1\right),
$$

which yields

$$
\|\mathbf{y}\|_{\mathbf{W}^{2, r}(\Omega)} \leq C\left(\|\mathbf{f}\|_{\mathbf{L}^{r}(\Omega)}^{4}+\|\mathbf{u}\|_{L^{r}\left(\omega ; \mathbb{R}^{m}\right)}^{4}+1\right)
$$


if $2 \leq r \leq 3$. Next we have

$$
\|(\mathbf{y} \cdot \nabla) \mathbf{y}\|_{\left(\mathbf{L}^{r}(\Omega)\right)^{d}} \leq C_{r}\|\mathbf{y}\|_{\mathbf{H}^{2}(\Omega)}\|\mathbf{y}\|_{\mathbf{W}^{2,3}(\Omega)} \leq C\left(\|\mathbf{f}\|_{\mathbf{L}^{2}(\Omega)}^{7}+\|\mathbf{u}\|_{L^{2}\left(\omega ; \mathbb{R}^{m}\right)}^{7}+1\right)
$$

if $3 \leq r<\infty$, which provides the desired estimate.

It is well known that the solution of (1.2) is unique when $\nu$ is large enough with respect to the right-hand side; see, for instance, Temam [20]. Since this is a strong assumption we are interested in the solutions of (1.2) for which the equation is locally unique. These solutions, called nonsingular solutions, are defined below.

DeFINITION 2.3. A function $\mathbf{y} \in \mathbf{V}_{0}^{1}(\Omega)$ is a nonsingular solution of (1.2), or equivalently (2.2), if $P\left(A+B^{\prime}(\mathbf{y})\right)$ is an isomorphism from $\mathbf{V}_{0}^{1}(\Omega)$ into $\mathbf{V}^{-1}(\Omega)$. If, moreover, $A \mathbf{y}+B(\mathbf{y})=\mathbf{f}+\mathcal{C} \mathbf{u}$ in $\mathbf{V}^{-1}(\Omega)$, with $\mathbf{u} \in L^{2}\left(\omega ; \mathbb{R}^{m}\right)$, we will also say that the pair $(\mathbf{u}, \mathbf{y})$ is a nonsingular solution of (1.2).

Remark 2.4. For a nonsingular solution $(\mathbf{u}, \mathbf{y})$ of $(1.2)$, the condition $P(A+$ $\left.B^{\prime}(\mathbf{y})\right) \in \operatorname{isom}\left(\mathbf{V}_{0}^{1}(\Omega), \mathbf{V}^{-1}(\Omega)\right)$ corresponds to the one stated in [12, Chapter 4, condition (3.4)], which is used to get the error estimates for the approximation of the Navier-Stokes equations.

The following theorem is a straightforward consequence of the implicit function theorem and will be useful in what follows.

THEOREM 2.5. Let $(\overline{\mathbf{u}}, \overline{\mathbf{y}}) \in L^{2}\left(\omega ; \mathbb{R}^{m}\right) \times \mathbf{V}_{0}^{1}(\Omega)$ be a nonsingular solution of $(1.2)$; then there exist an open neighborhood $\mathcal{O}(\overline{\mathbf{u}})$ of $\overline{\mathbf{u}}$ in $L^{2}\left(\omega ; \mathbb{R}^{m}\right)$, an open neighborhood $\mathcal{O}(\overline{\mathbf{y}})$ of $\overline{\mathbf{y}}$ in $\mathbf{V}_{0}^{1}(\Omega)$, and a mapping $G$ from $\mathcal{O}(\overline{\mathbf{u}})$ to $\mathcal{O}(\overline{\mathbf{y}})$ of class $C^{\infty}$ such that, for all $\mathbf{u} \in \mathcal{O}(\overline{\mathbf{u}}), G(\mathbf{u})=\mathbf{y}_{\mathbf{u}}$ is the unique solution in $\mathcal{O}(\overline{\mathbf{y}})$ to (2.2). Moreover, if $\mathbf{z}_{\mathbf{v}}=G^{\prime}(\mathbf{u}) \mathbf{v} \in \mathbf{V}_{0}^{1}(\Omega)$ and $\mathbf{w}=G^{\prime \prime}(\mathbf{u}) \mathbf{v}^{2} \in \mathbf{V}_{0}^{1}(\Omega)$, then $\mathbf{z}_{\mathbf{v}}$ and $\mathbf{w}$ satisfy the equations

$$
\begin{aligned}
A \mathbf{z}_{\mathbf{v}}+B^{\prime}\left(\mathbf{y}_{\mathbf{u}}\right) \mathbf{z}_{\mathbf{v}} & =\mathcal{C} \mathbf{v} \quad \text { in } \mathbf{V}^{-1}(\Omega), \\
A \mathbf{w}+B^{\prime}\left(\mathbf{y}_{\mathbf{u}}\right) \mathbf{w}+B^{\prime \prime}\left(\mathbf{z}_{\mathbf{v}}, \mathbf{z}_{\mathbf{v}}\right) & =0 \quad \text { in } \mathbf{V}^{-1}(\Omega),
\end{aligned}
$$

and $P\left(A+B^{\prime}\left(\mathbf{y}_{\mathbf{u}}\right)\right)$ is an isomorphism from $\mathbf{V}_{0}^{1}(\Omega)$ into $\mathbf{V}^{-1}(\Omega)$ for all $\mathbf{u} \in \mathcal{O}(\overline{\mathbf{u}})$.

Lemma 2.6. Let $(\overline{\mathbf{u}}, \overline{\mathbf{y}})$ be as in Theorem 2.5 , and let $\bar{p}$ be the associated pressure (the solution of (2.3) corresponding to $\overline{\mathbf{y}})$. Let $\left(\mathbf{u}_{k}\right)_{k}$ be a sequence in $\mathcal{O}(\overline{\mathbf{u}})$ weakly converging to $\overline{\mathbf{u}}$ in $L^{2}\left(\omega ; \mathbb{R}^{m}\right)$. Let $\mathbf{y}_{k}$ be the solution to (1.2) in $\mathcal{O}(\overline{\mathbf{y}})$ corresponding to $\mathbf{u}_{k}$, and let $p_{k}$ be the associated pressure. Then $\left(\mathbf{y}_{k}\right)_{k}$ converges to $\overline{\mathbf{y}}$ in $\mathbf{V}_{0}^{1}(\Omega)$, and $\left(p_{k}\right)_{k}$ converges to $\bar{p}$ in $L_{0}^{2}(\Omega)$.

Proof. The proof is an easy consequence of Theorem 2.2 and of formula (2.3).

3. Analysis of the control problem. The existence of a solution of problem (P) can be obtained by the usual approach of taking a minimizing sequence, which is bounded in $L^{2}\left(\omega ; \mathbb{R}^{m}\right) \times \mathbf{V}_{0}^{1}(\Omega)$, and passing to the limit; see, for instance, [18] for a detailed proof. In this section we will derive the first and second order optimality conditions for a local solution $(\overline{\mathbf{u}}, \overline{\mathbf{y}})$ in $U_{a d} \times \mathbf{V}_{0}^{1}(\Omega)$.

3.1. First order optimality conditions. Let us precisely define local solutions of $(\mathrm{P})$.

Definition 3.1. We shall say that $(\overline{\mathbf{u}}, \overline{\mathbf{y}}) \in U_{a d} \times \mathbf{V}_{0}^{1}(\Omega)$ is a local solution of $(\mathrm{P})$ if and only if $(\overline{\mathbf{u}}, \overline{\mathbf{y}})$ satisfies (1.2) and there exist neighborhoods $\mathcal{O}(\overline{\mathbf{u}})$ of $\overline{\mathbf{u}}$ in $L^{2}\left(\omega ; \mathbb{R}^{m}\right)$ and $\mathcal{O}(\overline{\mathbf{y}})$ of $\overline{\mathbf{y}}$ in $\mathbf{V}_{0}^{1}(\Omega)$ such that $F(\overline{\mathbf{u}}, \overline{\mathbf{y}}) \leq F(\mathbf{u}, \mathbf{y})$ for all pairs $(\mathbf{u}, \mathbf{y}) \in\left(U_{a d} \cap \mathcal{O}(\overline{\mathbf{u}})\right) \times \mathcal{O}(\overline{\mathbf{y}})$ satisfying $(1.2)$.

The following theorem was proved by Abergel and Casas [1] for a slightly different functional, but the proof can be repeated for our problem step by step, just by doing the obvious modifications. 
TheOrem 3.2. Let $(\overline{\mathbf{u}}, \overline{\mathbf{y}}) \in U_{a d} \times \mathbf{V}_{0}^{1}(\Omega)$ be a local solution of $(\mathrm{P})$; then there exist a real number $\bar{\lambda}$ and some elements $\overline{\mathbf{\Phi}} \in \mathbf{W}^{2, \bar{r}}(\Omega)$ and $\bar{\pi}, \bar{p} \in W^{1, \bar{r}}(\Omega)$ such that

$$
\begin{aligned}
& \bar{\lambda}+\|\overline{\mathbf{\Phi}}\|_{\mathbf{V}_{0}^{1}(\Omega)}>0, \\
& -\nu \Delta \overline{\mathbf{y}}+(\overline{\mathbf{y}} \cdot \nabla) \overline{\mathbf{y}}+\nabla \bar{p}=\mathbf{f}+\mathcal{C} \overline{\mathbf{u}} \text { in } \Omega, \operatorname{div} \overline{\mathbf{y}}=0 \text { in } \Omega, \overline{\mathbf{y}}=0 \text { on } \Gamma, \\
& -\nu \Delta \overline{\mathbf{\Phi}}+(\nabla \overline{\mathbf{y}})^{T} \overline{\mathbf{\Phi}}-(\overline{\mathbf{y}} \cdot \nabla) \overline{\mathbf{\Phi}}+\nabla \bar{\pi}=\bar{\lambda}\left(\overline{\mathbf{y}}-\mathbf{y}_{d}\right) \text { in } \Omega, \\
& \operatorname{div} \overline{\mathbf{\Phi}}=0 \text { in } \Omega, \overline{\mathbf{\Phi}}=0 \text { on } \Gamma, \\
& \int_{\omega}\left(\mathcal{C}^{*} \overline{\mathbf{\Phi}}+\bar{\lambda} N \overline{\mathbf{u}}\right) \cdot(\mathbf{u}-\overline{\mathbf{u}}) d x \geq 0 \forall \mathbf{u} \in U_{a d} .
\end{aligned}
$$

These conditions for optimality are of Fritz-John type, and we are interested in the cases where $\bar{\lambda}$ can be chosen equal to one. Gunzburger, Hou, and Svobodny [14] introduced an assumption on $U_{a d}$ for the local solution $(\overline{\mathbf{u}}, \overline{\mathbf{y}})$. The control set $U_{a d}$ is said to have the property $(C)$ at $(\overline{\mathbf{u}}, \overline{\mathbf{y}})$ if the system

$$
-\nu \Delta \boldsymbol{\Phi}+(\nabla \overline{\mathbf{y}})^{T} \boldsymbol{\Phi}-(\mathbf{y} \cdot \nabla) \boldsymbol{\Phi}+\nabla \pi=\bar{\lambda}\left(\overline{\mathbf{y}}-\mathbf{y}_{d}\right) \text { in } \Omega, \operatorname{div} \boldsymbol{\Phi}=0 \text { in } \Omega, \boldsymbol{\Phi}=0 \text { on } \Gamma,
$$

admits at least a nonzero solution $(\boldsymbol{\Phi}, \pi) \in \mathbf{V}_{0}^{1}(\Omega) \times L_{0}^{2}(\Omega)$, and if for any nonzero solution $(\boldsymbol{\Phi}, \pi)$ we can find $\mathbf{u} \in U_{a d}$ such that

$$
\int_{\omega} \mathcal{C}^{*} \boldsymbol{\Phi} \cdot(\mathbf{u}-\overline{\mathbf{u}}) d x<0
$$

It is obvious that if $U_{a d}$ has the property $(C)$ at $(\overline{\mathbf{u}}, \overline{\mathbf{y}})$, then $(3.2)-(3.5)$ hold with $\bar{\lambda}=1$.

Here we will make a different assumption which will be crucial in what follows, in particular for the numerical analysis. We consider only local solutions $(\overline{\mathbf{u}}, \overline{\mathbf{y}})$ of (P) such that $(\overline{\mathbf{u}}, \overline{\mathbf{y}})$ is a nonsingular solution of (2.2). In that case we shall say that $(\overline{\mathbf{u}}, \overline{\mathbf{y}})$ is a local nonsingular solution of $(\mathrm{P})$. For such a local nonsingular solution we can apply Theorem 2.5 and define the control problem

$\left(\mathrm{P}_{\mathcal{O}(\overline{\mathbf{u}})}\right)$

$$
\inf \left\{J(\mathbf{u}) \mid \mathbf{u} \in U_{a d} \cap \mathcal{O}(\overline{\mathbf{u}})\right\},
$$

where $J: \mathcal{U} \longmapsto \mathbb{R}$ is given by $J(\mathbf{u})=F(\mathbf{u}, G(\mathbf{u}))$. Then $\overline{\mathbf{u}}$ is a local solution of $\left(\mathrm{P}_{\mathcal{O}(\overline{\mathbf{u}})}\right)$. Let us study the differentiability properties of $J$.

TheOREM 3.3. Function $J$ is of class $C^{\infty}$ in $\mathcal{O}(\overline{\mathbf{u}})$, and for every $\mathbf{u} \in \mathcal{O}(\overline{\mathbf{u}})$ and $\mathbf{v} \in L^{2}\left(\omega ; \mathbb{R}^{m}\right)$ we have

$$
\begin{aligned}
& J^{\prime}(\mathbf{u}) \mathbf{v}=\int_{\omega}\left(\mathcal{C}^{*} \boldsymbol{\Phi}_{\mathbf{u}}+N \mathbf{u}\right) \cdot \mathbf{v} d x, \\
& J^{\prime \prime}(\mathbf{u}) \mathbf{v}^{2}=\int_{\Omega}\left(\left|\mathbf{z}_{\mathbf{v}}\right|^{2}-2\left(\mathbf{z}_{\mathbf{v}} \cdot \nabla\right) \mathbf{z}_{\mathbf{v}} \cdot \boldsymbol{\Phi}_{\mathbf{u}}\right) d x+N \int_{\omega}|\mathbf{v}|^{2} d x,
\end{aligned}
$$

where $\mathbf{z}_{\mathbf{v}}$ is the solution of (2.6) and $\mathbf{\Phi}_{\mathbf{u}} \in \mathbf{V}_{0}^{1}(\Omega)$ satisfies

$$
\left\{\begin{array}{l}
-\nu \Delta \boldsymbol{\Phi}_{\mathbf{u}}+\left(\nabla \mathbf{y}_{\mathbf{u}}\right)^{T} \boldsymbol{\Phi}_{\mathbf{u}}-\left(\mathbf{y}_{\mathbf{u}} \cdot \nabla\right) \boldsymbol{\Phi}_{\mathbf{u}}+\nabla \pi_{\mathbf{u}}=\mathbf{y}_{\mathbf{u}}-\mathbf{y}_{d} \text { in } \Omega \\
\operatorname{div} \boldsymbol{\Phi}_{\mathbf{u}}=0 \text { in } \Omega, \boldsymbol{\Phi}_{\mathbf{u}}=0 \text { on } \Gamma
\end{array}\right.
$$

The proof follows easily from Theorem 2.5. The only delicate point is the definition of $\boldsymbol{\Phi}_{\mathbf{u}}$. Let us remark that (3.8) is equivalent to the equation $A^{*} \boldsymbol{\Phi}_{\mathbf{u}}+$ 
$B^{\prime}\left(\mathbf{y}_{\mathbf{u}}\right)^{*} \boldsymbol{\Phi}_{\mathbf{u}}=\mathbf{y}_{\mathbf{u}}-\mathbf{y}_{d}$ in $\mathbf{V}^{-1}(\Omega)$, and due to Theorem 2.5 the operator $P\left(A^{*}+\right.$ $\left.B^{\prime}\left(\mathbf{y}_{\mathbf{u}}\right)^{*}\right)$ is an isomorphism from $\mathbf{V}_{0}^{1}(\Omega)$ into $\mathbf{V}^{-1}(\Omega)$.

By using the previous theorem we get the following result.

THEOREM 3.4. Let $(\overline{\mathbf{u}}, \overline{\mathbf{y}}) \in U_{a d} \times \mathbf{V}_{0}^{1}(\Omega)$ be a local nonsingular solution of $(\mathrm{P})$, and let $\bar{p}$ be the associated pressure; then there exist some elements $\overline{\mathbf{\Phi}} \in \mathbf{V}_{0}^{1}(\Omega)$ and $\bar{\pi} \in L_{0}^{2}(\Omega)$ such that (3.2)-(3.5) hold with $\bar{\lambda}=1$.

Proof. It is enough to take into account that $J^{\prime}(\overline{\mathbf{u}})(\mathbf{u}-\overline{\mathbf{u}}) \geq 0$ for all $\mathbf{u} \in U_{a d}$ and to use (3.6).

Using the first order necessary conditions we can deduce some extra regularity for the optimal control, the state, and the adjoint state.

TheOrem 3.5. Let $(\overline{\mathbf{u}}, \overline{\mathbf{y}})$ be a local nonsingular solution of $(\mathrm{P})$ and let $\overline{\mathbf{\Phi}}$ be the adjoint state as defined by (3.3)-(3.4) with $\bar{\lambda}=1$. Then $\overline{\mathbf{y}}, \overline{\mathbf{\Phi}} \in \mathbf{W}^{2, \bar{r}}(\Omega), \bar{p}, \bar{\pi} \in$ $W^{1, \bar{r}}(\Omega)$, and $\overline{\mathbf{u}} \in C^{0,1}\left(\bar{\omega} ; \mathbb{R}^{m}\right)$.

Proof. Taking into account that $\mathcal{C} \overline{\mathbf{u}} \in \mathbf{L}^{2}(\Omega)$ and the assumption on $\mathbf{f}$, it is enough to apply Theorem 2.2 to deduce that $\overline{\mathbf{y}}$ belongs to $\mathbf{H}^{2}(\Omega)$ and that $\overline{\mathbf{\Phi}}$ belongs to $\mathbf{W}^{2, \bar{r}}(\Omega)$. On the other hand, $\overline{\mathbf{\Phi}} \in \mathbf{W}^{2, \bar{r}}(\Omega) \subset C^{0,1}\left(\bar{\Omega} ; \mathbb{R}^{d}\right)$ because $\bar{r}>d$. Now using the Lipschitz property of the function $M$ defining $\mathcal{C}$ and the representation of the optimal control deduced from (3.5), we obtain

$$
\bar{u}_{j}(x)=\operatorname{Proj}_{\left[\alpha_{j}, \beta_{j}\right]}\left(-\frac{1}{N}\left(\mathcal{C}^{*} \overline{\mathbf{\Phi}}\right)_{j}(x)\right) \quad \text { for a.e. } x \in \omega,
$$

which gives the desired regularity for $\overline{\mathbf{u}}$. Now still using Theorem 2.2, we obtain the regularity of $\overline{\mathbf{y}}$.

3.2. Second order optimality conditions. To perform the numerical analysis of the problem as well as the analysis of the algorithms of optimization, second order sufficient conditions are required. These sufficient conditions should be as unrestrictive as possible. One way of measuring this is to compare them with the necessary second order conditions and check if the gap is small. This is the reason why we first introduce the second order necessary conditions.

Second order conditions have to be written for directions $\mathbf{v} \in T_{U_{a d}}(\overline{\mathbf{u}})$ such that $J^{\prime}(\mathbf{u}) \mathbf{v}=0$, where $T_{U_{a d}}(\overline{\mathbf{u}})$ is the tangent cone at $\overline{\mathbf{u}}$ to $U_{a d}$. To characterize these directions, we introduce $\overline{\mathbf{d}}(x)=\mathcal{C}^{*} \overline{\mathbf{\Phi}}(x)+N \overline{\mathbf{u}}(x)$ for $x \in \omega$, and the following conditions:

$$
\begin{aligned}
& v_{j}(x)=0 \text { if } \bar{d}_{j}(x) \neq 0, \\
& v_{j}(x) \geq 0 \text { if }-\infty<\alpha_{j}=\bar{u}_{j}(x) \text { and } \bar{d}_{j}(x)=0, \\
& v_{j}(x) \leq 0 \text { if } \bar{u}_{j}(x)=\beta_{j}<\infty \text { and } \bar{d}_{j}(x)=0 .
\end{aligned}
$$

Now we define the cone

$$
C_{\overline{\mathbf{u}}}=\left\{\mathbf{v} \in L^{2}\left(\omega ; \mathbb{R}^{m}\right) \mid \mathbf{v} \text { satisfies }(3.10)-(3.12)\right\} .
$$

Notice that

$$
\begin{aligned}
& J^{\prime}(\overline{\mathbf{u}}) \mathbf{v}=\int_{\omega} \overline{\mathbf{d}}(x) \cdot \mathbf{v}(x) d x \forall \mathbf{v} \in L^{2}\left(\omega ; \mathbb{R}^{m}\right), \\
& \overline{\mathbf{d}}(x) \cdot \mathbf{v}(x)=0 \text { for a.e. } x \in \omega \text { and all } \mathbf{v} \in C_{\overline{\mathbf{u}}} .
\end{aligned}
$$

TheOrEm 3.6. Let $(\overline{\mathbf{u}}, \overline{\mathbf{y}})$ be a nonsingular local solution of $(\mathrm{P})$. Then

$$
J^{\prime \prime}(\overline{\mathbf{u}}) \mathbf{v}^{2} \geq 0 \quad \forall \mathbf{v} \in C_{\overline{\mathbf{u}}} .
$$


Proof. We sketch the proof in the case where $-\infty<\alpha_{j}<\beta_{j}<\infty$ for all $1 \leq j \leq m$. The modifications for the other cases are obvious. Take $\mathbf{v} \in C_{\overline{\mathbf{u}}}$, and for $\varepsilon<\min \left\{\left(\beta_{j}-\alpha_{j}\right) / 2: 1 \leq j \leq m\right\}$ define

$$
v_{j, \varepsilon}(x)=\left\{\begin{array}{cl}
0 & \text { if } \alpha_{j}<\bar{u}_{j}(x)<\alpha_{j}+\varepsilon, \\
0 & \text { if } \beta_{j}-\varepsilon<\bar{u}_{j}(x)<\beta_{j}, \\
\operatorname{Proj}_{\left[-\frac{1}{\varepsilon}, \frac{1}{\varepsilon}\right]}\left(v_{j}(x)\right) & \text { otherwise. }
\end{array}\right.
$$

It is clear that $\left|v_{j, \varepsilon}(x)\right| \leq\left|v_{j}(x)\right|$ and that $v_{j, \varepsilon}(x) \rightarrow v_{j}(x)$ for a.e. $x \in \omega$ as $\varepsilon \rightarrow 0$, and hence $\mathbf{v}_{\varepsilon} \rightarrow \mathbf{v}$ in $L^{2}\left(\omega ; \mathbb{R}^{m}\right)$. A simple inspection convinces us that $\mathbf{v}_{\varepsilon} \in C_{\overline{\mathbf{u}}}$. Let us check that $\overline{\mathbf{u}}+\rho \mathbf{v}_{\varepsilon} \in U_{a d}$ for every $0<\rho<\varepsilon^{2}$. If $\bar{d}_{j}(x) \neq 0$, then $v_{j, \varepsilon}(x)=0$. So $\bar{u}_{j}(x)+\rho v_{j, \varepsilon}(x)=\bar{u}_{j}(x) \in\left[\alpha_{j}, \beta_{j}\right]$. For $\bar{d}_{j}(x)=0$, we have the following:

(1) If $\bar{u}_{j}(x)=\alpha_{j}$, then $v_{j}(x) \geq 0$ and $v_{j, \varepsilon}(x) \geq 0$. So clearly $\alpha_{j} \leq u_{j}(x)+\rho v_{j, \varepsilon}(x)$. For the other inequality we write $u_{j}(x)+\rho v_{j, \varepsilon}(x) \leq \alpha_{j}+\varepsilon^{2} \frac{1}{\varepsilon} \leq \frac{\alpha_{j}+\beta_{j}}{2}<\beta_{j}$. If $\bar{u}_{j}(x)=\beta_{j}$, the argument is completely analogous.

(2) If $\alpha_{j}<\bar{u}_{j}(x)<\alpha_{j}+\varepsilon$, then $\bar{u}_{j}(x)+\rho v_{j, \varepsilon}(x)=\bar{u}_{j}(x) \in\left[\alpha_{j}, \beta_{j}\right]$. The same applies if $\beta_{j}-\varepsilon<\bar{u}_{j}(x)<\beta_{j}$.

(3) If $\alpha_{j}+\varepsilon \leq \bar{u}_{j}(x) \leq \beta_{j}-\varepsilon$, then on the left side, $\bar{u}_{j}(x)+\rho v_{j, \varepsilon}(x) \geq \alpha_{j}+\varepsilon-\varepsilon^{2} \frac{1}{\varepsilon}=$ $\alpha_{j}$, and on the right side $\bar{u}_{j}(x)+\rho v_{j, \varepsilon}(x) \leq \beta_{j}-\varepsilon+\varepsilon^{2} \frac{1}{\varepsilon}=\beta_{j}$.

Thus $\overline{\mathbf{u}}+\rho \mathbf{v}_{\varepsilon}$ belongs to $U_{a d}$. Making a second order Taylor expansion of $J$ at $\overline{\mathbf{u}}$ and taking into account that it is a local minimum for $\rho<\varepsilon^{2}$ small enough, there exists $0<\theta_{\rho}<\rho$ such that

$$
0 \leq J\left(\overline{\mathbf{u}}+\rho \mathbf{v}_{\varepsilon}\right)-J(\overline{\mathbf{u}})=\rho J^{\prime}(\overline{\mathbf{u}}) \mathbf{v}_{\varepsilon}+\frac{\rho^{2}}{2} J^{\prime \prime}\left(\overline{\mathbf{u}}+\theta_{\rho} \mathbf{v}_{\varepsilon}\right) \mathbf{v}_{\varepsilon}^{2} .
$$

Since $\mathbf{v}_{\varepsilon} \in C_{\overline{\mathbf{u}}}$, (3.13) implies that $J^{\prime}(\overline{\mathbf{u}}) \mathbf{v}_{\varepsilon}=0$. Therefore the above inequality leads to $J^{\prime \prime}\left(\overline{\mathbf{u}}+\theta_{\rho} \mathbf{v}_{\varepsilon}\right) \mathbf{v}_{\varepsilon}^{2} \geq 0$. Now we must take the limit as $\rho \rightarrow 0$ to get $J^{\prime \prime}(\overline{\mathbf{u}}) \mathbf{v}_{\varepsilon}^{2} \geq 0$. Next it is enough to take the limit as $\varepsilon \rightarrow 0$. To do this, let us recall the expression of $J^{\prime \prime}(\overline{\mathbf{u}})$ provided by $(3.7)$ :

$$
\begin{aligned}
& J^{\prime \prime}(\overline{\mathbf{u}}) \mathbf{v}_{\varepsilon}^{2}=\int_{\Omega}\left(\left|\mathbf{z}_{\mathbf{v}_{\varepsilon}}\right|^{2}-2\left(\mathbf{z}_{\mathbf{v}_{\varepsilon}} \cdot \nabla\right) \mathbf{z}_{\mathbf{v}_{\varepsilon}} \cdot \boldsymbol{\Phi}_{\overline{\mathbf{u}}}\right) d x+N \int_{\omega}\left|\mathbf{v}_{\varepsilon}\right|^{2} d x \\
& \quad \longrightarrow \int_{\Omega}\left(\left|\mathbf{z}_{\mathbf{v}}\right|^{2}-2\left(\mathbf{z}_{\mathbf{v}} \cdot \nabla\right) \mathbf{z}_{\mathbf{v}} \cdot \boldsymbol{\Phi}_{\overline{\mathbf{u}}}\right) d x+N \int_{\omega}|\mathbf{v}|^{2} d x=J^{\prime \prime}(\overline{\mathbf{u}}) \mathbf{v}^{2} \quad \text { as } \varepsilon \rightarrow 0 .
\end{aligned}
$$

The following result is an obvious consequence of the previous theorem and the expression of $J^{\prime \prime}$ given by (3.7).

Corollary 3.7. Let $(\overline{\mathbf{u}}, \overline{\mathbf{y}})$ be a nonsingular local solution of $(\mathrm{P})$ and let $\overline{\mathbf{\Phi}}$ be the corresponding adjoint state. Then

$$
\int_{\Omega}\left(|\mathbf{z}|^{2}-2(\mathbf{z} \cdot \nabla) \mathbf{z} \cdot \overline{\mathbf{\Phi}}\right) d x+N \int_{\omega}|\mathbf{v}|^{2} d x \geq 0
$$

for every $(\mathbf{v}, \mathbf{z})$ satisfying the linearized state equation (2.6) and $\mathbf{v} \in C_{\overline{\mathbf{u}}}$.

To state second order sufficient conditions we will not suppose that $(\overline{\mathbf{u}}, \overline{\mathbf{y}})$ is a nonsingular solution of the Navier-Stokes equations (1.2). The result we are going to state is the following.

Theorem 3.8. Let $(\overline{\mathbf{u}}, \overline{\mathbf{y}}, \overline{\mathbf{\Phi}}) \in L^{2}\left(\omega ; \mathbb{R}^{m}\right) \times \mathbf{V}_{0}^{1}(\Omega) \times \mathbf{V}_{0}^{1}(\Omega)$ satisfy $(3.2)-(3.5)$ with $\bar{\lambda}=1$. Let us suppose that

$$
\int_{\Omega}\left(\mathbf{z}^{2}-2(\mathbf{z} \cdot \nabla) \mathbf{z} \cdot \overline{\mathbf{\Phi}}\right) d x+N \int_{\omega} \mathbf{v}^{2} d x>0
$$


for every $(\mathbf{v}, \mathbf{z}) \neq(0,0)$ satisfying the linearized state equation (2.6) and $\mathbf{v} \in C_{\overline{\mathbf{u}}}$. Then there exist $\varepsilon>0$ and $\mu>0$ such that

$$
F(\overline{\mathbf{u}}, \overline{\mathbf{y}})+\frac{\mu}{2}\left(\|\mathbf{u}-\overline{\mathbf{u}}\|_{L^{2}\left(\omega ; \mathbb{R}^{m}\right)}^{2}+\|\mathbf{y}-\overline{\mathbf{y}}\|_{\mathbf{L}^{2}(\Omega)}^{2}\right) \leq F(\mathbf{u}, \mathbf{y})
$$

for every $(\mathbf{u}, \mathbf{y})$ satisfying $(1.2), \mathbf{u} \in U_{a d}$, and $\|\mathbf{u}-\overline{\mathbf{u}}\|_{L^{2}\left(\omega ; \mathbb{R}^{m}\right)}^{2}+\|\mathbf{y}-\overline{\mathbf{y}}\|_{\mathbf{L}^{2}(\Omega)}^{2} \leq \varepsilon^{2}$.

Proof. Let us suppose the theorem is false. In that case, for all $k \in \mathbb{N}$, there exists $\left(\mathbf{u}_{k}, \mathbf{y}_{k}\right)$ satisfying $(1.2), \mathbf{u}_{k} \in U_{a d}$,

$$
\left\|\mathbf{u}_{k}-\overline{\mathbf{u}}\right\|_{L^{2}\left(\omega ; \mathbb{R}^{m}\right)}^{2}+\left\|\mathbf{y}_{k}-\overline{\mathbf{y}}\right\|_{\mathbf{L}^{2}(\Omega)}^{2}<\frac{1}{k^{2}},
$$

and

$$
F(\overline{\mathbf{u}}, \overline{\mathbf{y}})+\frac{1}{k}\left(\left\|\mathbf{u}_{k}-\overline{\mathbf{u}}\right\|_{L^{2}\left(\omega ; \mathbb{R}^{m}\right)}^{2}+\left\|\mathbf{y}_{k}-\overline{\mathbf{y}}\right\|_{\mathbf{L}^{2}(\Omega)}^{2}\right)>F\left(\mathbf{u}_{k}, \mathbf{y}_{k}\right) .
$$

Since the sequence $\left\{\mathbf{u}_{k}\right\}_{k=1}^{\infty}$ is bounded in $L^{2}\left(\omega ; \mathbb{R}^{m}\right)$, Theorem 2.2 implies that $\left\{\mathbf{y}_{k}\right\}_{k=1}^{\infty}$ is bounded in $\mathbf{H}^{2}(\Omega) \cap \mathbf{V}_{0}^{1}(\Omega)$. Let us set

$$
\rho_{k}=\sqrt{\left\|\mathbf{u}_{k}-\overline{\mathbf{u}}\right\|_{L^{2}\left(\omega ; \mathbb{R}^{m}\right)}^{2}+\left\|\mathbf{y}_{k}-\overline{\mathbf{y}}\right\|_{\mathbf{L}^{2}(\Omega)}^{2}}, \mathbf{v}_{k}=\frac{\mathbf{u}_{k}-\overline{\mathbf{u}}}{\rho_{k}}, \mathbf{z}_{k}=\frac{\mathbf{y}_{k}-\overline{\mathbf{y}}}{\rho_{k}} .
$$

Clearly $\left\|\mathbf{v}_{k}\right\|_{L^{2}\left(\omega ; \mathbb{R}^{m}\right)}^{2}+\left\|\mathbf{z}_{k}\right\|_{\mathbf{L}^{2}(\Omega)}^{2}=1$, and hence there exist weakly convergent subsequences in $L^{2}\left(\omega ; \mathbb{R}^{m}\right)$ and $\mathbf{L}^{2}(\Omega)$, still indexed by $k$, such that $\mathbf{v}_{k} \rightarrow \mathbf{v}, \mathbf{z}_{k} \rightarrow \mathbf{z}$. We are going to check that the pair $(\mathbf{v}, \mathbf{z})$ satisfies the linearized equation (2.6) and $\mathbf{v} \in C_{\overline{\mathbf{u}}}$.

The pair $\left(\mathbf{v}_{k}, \mathbf{z}_{k}\right)$ satisfies the equation

$$
\left\{\begin{array}{l}
-\nu \Delta \mathbf{z}_{k}+(\overline{\mathbf{y}} \cdot \nabla) \mathbf{z}_{k}+\left(\mathbf{z}_{k} \cdot \nabla\right) \mathbf{y}_{k}+\nabla \pi_{k}=\mathcal{C} \mathbf{v}_{k} \text { in } \Omega, \\
\operatorname{div} \mathbf{z}_{k}=0 \text { in } \Omega, \mathbf{z}_{k}=0 \text { on } \Gamma,
\end{array}\right.
$$

where $\pi_{k}=\left(\bar{p}-p_{k}\right) / \rho_{k}$, which is equivalent to the variational formulation

$$
a\left(\mathbf{z}_{k}, \mathbf{z}\right)+b\left(\overline{\mathbf{y}}, \mathbf{z}_{k}, \mathbf{z}\right)+b\left(\mathbf{z}_{k}, \mathbf{y}_{k}, \mathbf{z}\right)=\left(\mathcal{C} \mathbf{v}_{k}, \mathbf{z}\right) \forall \mathbf{z} \in \mathbf{V}_{0}^{1}(\Omega) .
$$

Taking $\mathbf{z}=\mathbf{z}_{k}$ and using (2.4), we obtain

$$
a\left(\mathbf{z}_{k}, \mathbf{z}_{k}\right)=\left(\mathcal{C} \mathbf{v}_{k}, \mathbf{z}_{k}\right)-\int_{\Omega}\left(\mathbf{z}_{k} \cdot \nabla\right) \mathbf{y}_{k} \cdot \mathbf{z}_{k} d x
$$

Using the equality $\left\|\mathbf{v}_{k}\right\|_{L^{2}\left(\omega ; \mathbb{R}^{m}\right)}^{2}+\left\|\mathbf{z}_{k}\right\|_{\mathbf{L}^{2}(\Omega)}^{2}=1$ and the imbedding $\mathbf{H}^{1}(\Omega) \subset \mathbf{L}^{4}(\Omega)$, we obtain

$$
\nu\left\|\mathbf{z}_{k}\right\|_{\mathbf{H}^{1}(\Omega)}^{2} \leq\|\mathcal{C}\|+\left\|\mathbf{y}_{k}\right\|_{\mathbf{H}^{1}(\Omega)}\left\|\mathbf{z}_{k}\right\|_{\mathbf{L}^{4}(\Omega)}^{2} \leq C\left(1+\left\|\mathbf{z}_{k}\right\|_{\mathbf{L}^{4}(\Omega)}^{2}\right),
$$

because $\left(\mathbf{y}_{k}\right)_{k}$ is bounded in $\mathbf{H}^{1}(\Omega)$. From the well-known interpolation inequality when $d=3$ (see Temam [20, Lemma 3.5, p. 296]),

$$
\|\zeta\|_{L^{4}(\Omega)} \leq \sqrt{2}\|\zeta\|_{L^{2}(\Omega)}^{1 / 4}\|\zeta\|_{H^{1}(\Omega)}^{3 / 4} \quad \forall \zeta \in H_{0}^{1}(\Omega),
$$

and the bound $\left\|\mathbf{z}_{k}\right\|_{\mathbf{L}^{2}(\Omega)} \leq 1$, it follows that

$$
\left\|\mathbf{z}_{k}\right\|_{\mathbf{H}^{1}(\Omega)}^{2} \leq C\left(1+\left\|\mathbf{z}_{k}\right\|_{\mathbf{H}^{1}(\Omega)}^{3 / 2}\right) .
$$


Thus the sequence $\left\{\mathbf{z}_{k}\right\}$ is bounded in $\mathbf{V}_{0}^{1}(\Omega)$, and therefore $\left\{\mathbf{z}_{k}\right\}$ converges strongly to $\mathbf{z}$ in $\mathbf{L}^{2}(\Omega)$. Now we can take the limit in (3.18), and we obtain that $(\mathbf{v}, \mathbf{z})$ satisfies (2.6).

Let us now check that $\mathbf{v} \in C_{\overline{\mathbf{u}}}$. The sign condition (3.11)-(3.12) is satisfied by $v_{k, j}$, and this is conserved when we pass to the weak limit because the set of functions satisfying these sign conditions is closed and convex in $L^{2}\left(\omega ; \mathbb{R}^{m}\right)$. On the other hand, using condition (3.16), for all $k$, we have

$$
\begin{aligned}
\frac{\rho_{k}}{k} & >\frac{F\left(\overline{\mathbf{u}}+\rho_{k} \mathbf{v}_{k}, \overline{\mathbf{y}}+\rho_{k} \mathbf{z}_{k}\right)-F(\overline{\mathbf{u}}, \overline{\mathbf{y}})}{\rho_{k}} \\
& =\frac{1}{2} \int_{\Omega} \frac{\left|\overline{\mathbf{y}}+\rho_{k} \mathbf{z}_{k}-\mathbf{y}_{d}\right|^{2}-\left|\overline{\mathbf{y}}-\mathbf{y}_{d}\right|^{2}}{\rho_{k}} d x+\frac{N}{2} \int_{\omega} \frac{\left|\overline{\mathbf{u}}+\rho_{k} \mathbf{v}_{k}\right|^{2}-|\overline{\mathbf{u}}|^{2}}{\rho_{k}} d x \\
& =\frac{1}{2} \int_{\Omega}\left(2\left(\overline{\mathbf{y}}-\mathbf{y}_{d}\right)+\rho_{k} \mathbf{z}_{k}\right) \cdot \mathbf{z}_{k} d x+\frac{N}{2} \int_{\omega}\left(2 \overline{\mathbf{u}}+\rho_{k} \mathbf{v}_{k}\right) \cdot \mathbf{v}_{k} d x .
\end{aligned}
$$

Since $\left\|\mathbf{v}_{k}\right\|_{L^{2}\left(\omega ; \mathbb{R}^{m}\right)}^{2}+\left\|\mathbf{z}_{k}\right\|_{\mathbf{L}^{2}(\Omega)}^{2}=1, \rho_{k}<1 / k$ converges to $0, \mathbf{z}_{k} \rightarrow \mathbf{z}$ in $\mathbf{L}^{2}(\Omega)$, and $\mathbf{v}_{k} \rightarrow \mathbf{v}$ weakly in $L^{2}\left(\omega ; \mathbb{R}^{m}\right)$, we can pass to the limit when $k$ tends to infinity, and we get

$$
\int_{\Omega}\left(\overline{\mathbf{y}}-\mathbf{y}_{d}\right) \cdot \mathbf{z} d x+N \int_{\omega} \overline{\mathbf{u}} \cdot \mathbf{v} d x \leq 0
$$

which is exactly

$$
\int_{\omega} \overline{\mathbf{d}}(x) \cdot \mathbf{v}(x) \leq 0
$$

The sign condition (3.5) implies that $\bar{d}_{j}(x) v_{j}(x) \geq 0$ for a.e. $x \in \omega$; therefore the above inequality is equivalent to

$$
\sum_{j=1}^{m} \int_{\Omega}\left|\bar{d}_{j}(x) v_{j}(x)\right| d x \leq 0 .
$$

Thus if $\bar{d}_{j}(x) \neq 0, v_{j}(x)=0,1 \leq j \leq m$, and hence $\mathbf{v} \in C_{\overline{\mathbf{u}}}$.

Making a second order Taylor expansion of $F$ at $(\overline{\mathbf{u}}, \overline{\mathbf{y}})$, with condition (3.16), we obtain

$$
\frac{1}{\rho_{k}}\left(\partial_{\mathbf{u}} F(\overline{\mathbf{u}}, \overline{\mathbf{y}}) \mathbf{v}_{k}+\partial_{\mathbf{y}} F(\overline{\mathbf{u}}, \overline{\mathbf{y}}) \mathbf{z}_{k}\right)+\frac{1}{2} \int_{\Omega}\left|\mathbf{z}_{k}\right|^{2} d x+\frac{N}{2} \int_{\omega}\left|\mathbf{v}_{k}\right|^{2} d x<\frac{1}{k} .
$$

Notice that the pair $\left(\mathbf{v}_{k}, \mathbf{z}_{k}\right)$ satisfies (3.17), but does not satisfy the linearized equation (2.6). Thus $\frac{1}{\rho_{k}}\left(\partial_{\mathbf{u}} F(\overline{\mathbf{u}}, \overline{\mathbf{y}}) \mathbf{v}_{k}+\partial_{\mathbf{y}} F(\overline{\mathbf{u}}, \overline{\mathbf{y}}) \mathbf{z}_{k}\right)$ is not equal to $\int_{\omega} \overline{\mathbf{d}}(x) \cdot \mathbf{v}_{k}(x) d x$. We can write (3.17) as follows:

$$
\begin{aligned}
& -\nu \Delta \mathbf{z}_{k}+(\overline{\mathbf{y}} \cdot \nabla) \mathbf{z}_{k}+\left(\mathbf{z}_{k} \cdot \nabla\right) \overline{\mathbf{y}}+\nabla \pi_{k}=\mathcal{C} \mathbf{v}_{k}-\left(\mathbf{z}_{k} \cdot \nabla\right)\left(\mathbf{y}_{k}-\overline{\mathbf{y}}\right) \text { in } \Omega, \\
& \operatorname{div} \mathbf{z}_{k}=0 \text { in } \Omega, \mathbf{z}_{k}=0 \text { on } \Gamma .
\end{aligned}
$$

Since

$$
\partial_{\mathbf{u}} F(\overline{\mathbf{u}}, \overline{\mathbf{y}}) \mathbf{v}_{k}+\partial_{\mathbf{y}} F(\overline{\mathbf{u}}, \overline{\mathbf{y}}) \mathbf{z}_{k}=\int_{\Omega}\left(\overline{\mathbf{y}}-\mathbf{y}_{d}\right) \cdot \mathbf{z}_{k} d x+N \int_{\omega} \overline{\mathbf{u}} \cdot \mathbf{v}_{k} d x
$$


using the adjoint state $\overline{\mathbf{\Phi}}$ and making an integration by parts, we get that

$$
\int_{\Omega}\left(\overline{\mathbf{y}}-\mathbf{y}_{d}\right) \cdot \mathbf{z}_{k} d x=\int_{\Omega} \overline{\mathbf{\Phi}} \cdot\left(\mathcal{C} \mathbf{v}_{k}-\left(\mathbf{z}_{k} \cdot \nabla\right)\left(\mathbf{y}_{k}-\overline{\mathbf{y}}\right)\right) d x
$$

and therefore

$$
\frac{1}{\rho_{k}}\left(\partial_{\mathbf{u}} F(\overline{\mathbf{u}}, \overline{\mathbf{y}}) \mathbf{v}_{k}+\partial_{\mathbf{y}} F(\overline{\mathbf{u}}, \overline{\mathbf{y}}) \mathbf{z}_{k}\right)=\frac{1}{\rho_{k}} \int_{\omega} \overline{\mathbf{d}}(x) \cdot \mathbf{v}_{k}(x) d x-\int_{\Omega}\left(\mathbf{z}_{k} \cdot \nabla\right) \mathbf{z}_{k} \cdot \overline{\mathbf{\Phi}} d x .
$$

Since $\mathbf{v}_{k}$ satisfy the sign condition, we have $\overline{\mathbf{d}}(x) \cdot \mathbf{v}_{k}(x) \geq 0$; therefore (3.19) leads to

$$
-2 \int_{\Omega}\left(\mathbf{z}_{k} \cdot \nabla\right) \mathbf{z}_{k} \cdot \overline{\mathbf{\Phi}} d x+\int_{\Omega}\left|\mathbf{z}_{k}\right|^{2} d x+N \int_{\omega}\left|\mathbf{v}_{k}\right|^{2} d x<\frac{2}{k} \quad \forall k .
$$

Taking the inferior limit in this inequality we deduce

$$
\int_{\Omega}\left(|\mathbf{z}|^{2}-2(\mathbf{z} \cdot \nabla) \mathbf{z} \cdot \overline{\mathbf{\Phi}}\right) d x+N \int_{\omega}|\mathbf{v}|^{2} d x \leq 0 .
$$

Since $\mathbf{v} \in C_{\overline{\mathbf{u}}}$ and the pair $(\mathbf{v}, \mathbf{z})$ satisfies the linearized equation (2.6), this is possible only if $(\mathbf{v}, \mathbf{z})=(0,0)$.

The sequence $\left\{\mathbf{z}_{k}\right\}_{k=1}^{\infty}$ converges strongly in $\mathbf{L}^{2}(\Omega)$ and weakly in $\mathbf{V}_{0}^{1}(\Omega)$. Since $\overline{\mathbf{\Phi}} \in \mathbf{L}^{\infty}(\Omega)$, by passing to the limit when $k$ tends to infinity, we obtain

$$
-2 \int_{\Omega}\left(\mathbf{z}_{k} \cdot \nabla\right) \mathbf{z}_{k} \cdot \overline{\mathbf{\Phi}} d x+\int_{\Omega}\left|\mathbf{z}_{k}\right|^{2} d x \rightarrow-2 \int_{\Omega}(\mathbf{z} \cdot \nabla) \mathbf{z} \cdot \overline{\mathbf{\Phi}} d x+\int_{\Omega}|\mathbf{z}|^{2} d x=0 .
$$

The last three relations imply that $\mathbf{v}_{k} \rightarrow 0$ strongly in $L^{2}\left(\omega ; \mathbb{R}^{m}\right)$. So we have proved that $\left(\mathbf{v}_{k}, \mathbf{z}_{k}\right) \rightarrow 0$ strongly in $L^{2}(\omega)^{m} \times \mathbf{L}^{2}(\Omega)$, which contradicts the fact that

$$
\left\|\mathbf{v}_{\mathbf{k}}\right\|_{L^{2}\left(\omega ; \mathbb{R}^{m}\right)}^{2}+\left\|\mathbf{z}_{\mathbf{k}}\right\|_{\mathbf{L}^{2}(\Omega)}^{2}=1
$$

The proof is complete.

The sufficient condition (3.15) is the best possible. Actually the gap between (3.15) and the second order necessary condition (3.14) is the same as in finite dimension. In the case of nonsingular solutions we have the following result analogous to Theorem 3.6.

COROLlaRY 3.9. Let us assume that $(\overline{\mathbf{u}}, \overline{\mathbf{y}})$ is a nonsingular solution of (1.2) and $(\overline{\mathbf{u}}, \overline{\mathbf{y}}, \overline{\mathbf{\Phi}})$ satisfies $(3.2)-(3.5)$ with $\bar{\lambda}=1$. Then (3.15) is equivalent to $J^{\prime \prime}(\overline{\mathbf{u}}) \mathbf{v}^{2}>0$ for every $\mathbf{v} \in C_{\overline{\mathbf{u}}} \backslash\{0\}$.

This corollary is an immediate consequence of (3.7) and the fact that $\mathbf{z}=\mathbf{z}_{\mathbf{v}}$ if $(\mathbf{v}, \mathbf{z})$ satisfies $(2.6)$.

To make the numerical analysis of control problem $(\mathrm{P})$, we will use the following equivalent condition to (3.15), which may seem stronger but is not, as we will see below. Given $\tau>0$, let us define a bigger cone than $C_{\overline{\mathbf{u}}}$ in the following way:

$$
\begin{aligned}
& v_{j}(x)=0 \text { if }\left|\bar{d}_{j}(x)\right|>\tau, \\
& v_{j}(x) \geq 0 \text { if }-\infty<\alpha_{j}=\bar{u}_{j}(x) \text { and }\left|\bar{d}_{j}(x)\right| \leq \tau, \\
& v_{j}(x) \leq 0 \text { if } \bar{u}_{j}(x)=\beta_{j}<\infty \text { and }\left|\bar{d}_{j}(x)\right| \leq \tau,
\end{aligned}
$$

and

$$
C_{\overline{\mathbf{u}}}^{\tau}=\left\{\mathbf{v} \in L^{2}\left(\omega ; \mathbb{R}^{m}\right) \mid \mathbf{v} \text { satisfies }(3.20)-(3.22)\right\}
$$


Theorem 3.10. Let $(\overline{\mathbf{u}}, \overline{\mathbf{y}}, \overline{\mathbf{\Phi}}) \in L^{2}\left(\omega ; \mathbb{R}^{m}\right) \times \mathbf{V}_{0}^{1}(\Omega) \times \mathbf{V}_{0}^{1}(\Omega)$ satisfy (3.2)-(3.5) with $\bar{\lambda}=1$. Then the condition (3.15) is equivalent to the existence of $\delta>0$ and $\tau>0$ such that

$$
\int_{\Omega}\left(|\mathbf{z}|^{2}-2(\mathbf{z} \cdot \nabla) \mathbf{z} \cdot \overline{\mathbf{\Phi}}\right) d x+N \int_{\omega}|\mathbf{v}|^{2} d x \geq \delta\left(\|\mathbf{v}\|_{L^{2}\left(\omega ; \mathbb{R}^{m}\right)}^{2}+\|\mathbf{z}\|_{\mathbf{L}^{2}(\Omega)}^{2}\right)
$$

for every $(\mathbf{v}, \mathbf{z})$ satisfying the linearized state equation (2.6) and $\mathbf{v} \in C_{\overline{\mathbf{u}}}^{\tau}$.

Proof. Notice that $C_{\overline{\mathbf{u}}}=C_{\overline{\mathbf{u}}}^{0} \subseteq C_{\overline{\mathbf{u}}}^{\tau}$; therefore (3.23) implies (3.15).

Suppose that (3.15) holds and (3.23) is false. In that case, for every $k \in \mathbb{N}$ there exists a pair $\left(\mathbf{v}_{k}, \mathbf{z}_{k}\right)$ satisfying the linearized state equation (2.6), $\mathbf{v}_{k} \in C_{\overline{\mathbf{u}}}^{1 / k}$, and

$$
\int_{\Omega}\left(\left|\mathbf{z}_{k}\right|^{2}-2\left(\mathbf{z}_{k} \cdot \nabla\right) \mathbf{z}_{k} \cdot \overline{\mathbf{\Phi}}\right) d x+N \int_{\omega} \mathbf{v}_{k}^{2} d x<\frac{1}{k}\left(\left\|\mathbf{v}_{k}\right\|_{L^{2}\left(\omega ; \mathbb{R}^{m}\right)}^{2}+\left\|\mathbf{z}_{k}\right\|_{\mathbf{L}^{2}(\Omega)}^{2}\right)
$$

We can suppose that $\left\|\mathbf{v}_{k}\right\|_{L^{2}\left(\omega ; \mathbb{R}^{m}\right)}^{2}+\left\|\mathbf{z}_{k}\right\|_{\mathbf{L}^{2}(\Omega)}^{2}=1$; otherwise we can redefine $\mathbf{v}_{k}=$ $\mathbf{v}_{k} / \rho_{k}$ and $\mathbf{z}_{k}=\mathbf{z}_{k} / \rho_{k}$, with $\rho_{k}=\left(\left\|\mathbf{v}_{k}\right\|_{L^{2}\left(\omega ; \mathbb{R}^{m}\right)}^{2}+\left\|\mathbf{z}_{k}\right\|_{\mathbf{L}^{2}(\Omega)}^{2}\right)^{1 / 2}$. Then there exist two weakly convergent subsequences in $L^{2}\left(\omega ; \mathbb{R}^{m}\right)$ and $\mathbf{L}^{2}(\Omega)$, still indexed by $k$, such that $\mathbf{v}_{k} \rightarrow \mathbf{v}$ and $\mathbf{z}_{k} \rightarrow \mathbf{z}$. Repeating the argument of the proof of Theorem 3.8, we deduce that the pair $(\mathbf{v}, \mathbf{z})$ satisfies the linearized equation $(2.6)$ and $\left\{\mathbf{z}_{k}\right\}_{k=1}^{\infty}$ is bounded in $\mathbf{V}_{0}^{1}(\Omega)$. Thus $\left\{\mathbf{z}_{k}\right\}_{k=1}^{\infty}$ converges strongly in $\mathbf{L}^{4}(\Omega)$. Let us prove that $\mathbf{v} \in C_{\overline{\mathbf{u}}}$. The sign condition (3.11)-(3.12) is again trivial since every $\mathbf{v}_{k}$ satisfies it. To check condition (3.10) we are going to prove that if $\left|\bar{d}_{j}(x)\right| \neq 0$, then $v_{j}(x)=0$. Let us fix $\varepsilon>0$ and define $\omega_{\varepsilon}=\left\{x \in \omega:\left|d_{j}(x)\right|>\varepsilon\right\}$. Notice that $\int_{\omega_{\varepsilon}} v_{j, k}(x) \bar{d}_{j}(x) d x \rightarrow$ $\int_{\omega_{\varepsilon}} v_{j}(x) \bar{d}_{j}(x) d x$ when $k$ tends to infinity. From the definition of $C_{\overline{\mathbf{u}}}^{1 / k}$ it follows that for $k>1 / \varepsilon$ all the terms of the sequence $\left\{\int_{\omega_{\varepsilon}} v_{j, k}(x) \bar{d}_{j}(x) d x\right\}_{k}$ are 0 , and so the limit is also 0 . Since $\mathbf{v}$ satisfies the sign condition (3.5), this can happen only if $v_{j}(x)=0$ almost everywhere in $\omega_{\varepsilon}$. Since $\varepsilon$ is arbitrarily small, we conclude that $v_{j}(x)=0$ for a.e. $x$ such that $\left|\bar{d}_{j}(x)\right| \neq 0$, and so $\mathbf{v} \in C_{\overline{\mathbf{u}}}$.

Finally, taking the lower limit in (3.24) we obtain that

$$
\int_{\Omega}\left(\mathbf{z}^{2}-2(\mathbf{z} \cdot \nabla) \mathbf{z} \cdot \overline{\mathbf{\Phi}}\right) d x+N \int_{\omega} \mathbf{v}^{2} d x \leq 0
$$

We complete the proof by arguing as at the end of the proof of Theorem 3.8.

COROLlary 3.11. Let us assume that $(\overline{\mathbf{u}}, \overline{\mathbf{y}})$ is a nonsingular solution of (1.2) and $(\overline{\mathbf{u}}, \overline{\mathbf{y}}, \overline{\mathbf{\Phi}})$ satisfies $(3.2)-(3.5)$ with $\bar{\lambda}=1$. Then (3.15) is equivalent to the existence of $\delta>0$ and $\tau>0$ such that

$$
J^{\prime \prime}(\overline{\mathbf{u}}) \mathbf{v}^{2} \geq \delta\|\mathbf{v}\|_{L^{2}\left(\omega ; \mathbb{R}^{m}\right)}^{2} \forall \mathbf{v} \in C_{\overline{\mathbf{u}}}^{\tau}
$$

This a consequence of Theorem 3.10 and the expression of $J^{\prime \prime}(\overline{\mathbf{u}})$ stated in (3.7). 


\section{Numerical approximation of the control problem.}

4.1. Numerical analysis of the state equation. Let $\mathbf{X}_{h} \subset \mathbf{H}_{0}^{1}(\Omega)$ and $M_{h} \subset$ $L_{0}^{2}(\Omega)$ be two finite dimensional spaces satisfying the assumptions (H1)-(H3) stated below.

(H1) (Approximation property of $\left.\mathbf{X}_{h}\right)$. There exists an operator $r_{h} \in \mathcal{L}\left(\mathbf{H}^{2}(\Omega) \cap\right.$ $\left.\mathbf{H}_{0}^{1}(\Omega), \mathbf{X}_{h}\right)$ such that

(a) $\left\|\mathbf{y}-r_{h} \mathbf{y}\right\|_{\mathbf{H}_{0}^{1}(\Omega)} \leq C h\|\mathbf{y}\|_{\mathbf{H}^{2}(\Omega)} \forall \mathbf{y} \in \mathbf{H}^{2}(\Omega) \cap \mathbf{H}_{0}^{1}(\Omega)$,

(b) $\left\|\mathbf{y}-r_{h} \mathbf{y}\right\|_{\mathbf{L}^{2}(\Omega)} \leq C h^{2}\|\mathbf{y}\|_{\mathbf{H}^{2}(\Omega)} \forall \mathbf{y} \in \mathbf{H}^{2}(\Omega) \cap \mathbf{H}_{0}^{1}(\Omega)$,

(c) $\left\|\mathbf{y}-r_{h} \mathbf{y}\right\|_{\mathbf{L}^{\infty}(\Omega)} \leq C h^{2-d / 2}\|\mathbf{y}\|_{\mathbf{H}^{2}(\Omega)} \forall \mathbf{y} \in \mathbf{H}^{2}(\Omega) \cap \mathbf{H}_{0}^{1}(\Omega)$,

(d) $\left\|\mathbf{y}_{h}\right\|_{\mathbf{L}^{\infty}(\Omega)} \leq C h^{-d / 2}\left\|\mathbf{y}_{h}\right\|_{\mathbf{L}^{2}(\Omega)} \forall \mathbf{y}_{h} \in \mathbf{X}_{h}$.

(H2) (Approximation property of $\left.M_{h}\right)$. There exists an operator $s_{h} \in \mathcal{L}\left(L_{0}^{2}(\Omega), M_{h}\right)$ such that

$$
\left\|p-s_{h} p\right\|_{L_{0}^{2}(\Omega)} \leq C h\|p\|_{H^{1}(\Omega)} \forall p \in H^{1}(\Omega) \cap L_{0}^{2}(\Omega) .
$$
that

(H3) (Uniform inf-sup condition). For each $p_{h} \in M_{h}$ there exists $\mathbf{y}_{h} \in \mathbf{X}_{h}$ such

$$
\left(p_{h}, \operatorname{div} \mathbf{y}_{h}\right)=\left\|p_{h}\right\|_{L_{0}^{2}(\Omega)}^{2} \quad \text { and } \quad\left\|\mathbf{y}_{h}\right\|_{\mathbf{H}_{0}^{1}(\Omega)} \leq C\left\|p_{h}\right\|_{L_{0}^{2}(\Omega)},
$$

where $C>0$ is independent of $h, p_{h}$, and $\mathbf{y}_{h}$.

Remark 4.1. Assumptions (H1)(b), (H1)(c), and (H1)(d) are needed to establish uniform convergence for the approximation of the state and the adjoint state (cf. Lemmas 4.10 and 4.13). In particular, if we use the finite element method when the family of triangulations is quasi-uniform, the above assumptions are satisfied for the Taylor-Hood finite element method and for the (P1-Bubble, P1) finite element method (see [12, p. 98, Lemma A.7 on p. 103, and Chapter 2]). The quasi-uniformity condition can be relaxed in some cases. For instance, Eriksson [11] gives some conditions on a locally refined family of triangulations in order to have an inverse inequality similar to $(\mathrm{H} 1)(\mathrm{d})$.

Assumption (H3) is equivalent to the classical inf-sup condition. See GiraultRaviart [12, Remark II.1.4].

For $\rho>0, \overline{\mathbf{y}} \in \mathbf{H}_{0}^{1}(\Omega), \bar{p} \in L_{0}^{2}(\Omega)$, and $\overline{\mathbf{u}} \in L^{2}\left(\omega ; \mathbb{R}^{m}\right)$, let us set

$$
\begin{aligned}
& B_{\rho}(\overline{\mathbf{y}})=\left\{\mathbf{y} \in \mathbf{H}_{0}^{1}(\Omega) \mid\|\mathbf{y}-\overline{\mathbf{y}}\|_{\mathbf{H}_{0}^{1}(\Omega)} \leq \rho\right\}, \\
& B_{\rho}(\bar{p})=\left\{p \in L_{0}^{2}(\Omega) \mid\|p-\bar{p}\|_{L_{0}^{2}(\Omega)} \leq \rho\right\}, \\
& B_{\rho}(\overline{\mathbf{u}})=\left\{\mathbf{u} \in L^{2}\left(\omega ; \mathbb{R}^{m}\right) \mid\|\mathbf{u}-\overline{\mathbf{u}}\|_{L^{2}\left(\omega ; \mathbb{R}^{m}\right)} \leq \rho\right\} .
\end{aligned}
$$

For all $\mathbf{u} \in L^{2}\left(\omega ; \mathbb{R}^{m}\right)$, we define a discrete state equation in $\mathbf{X}_{h} \times M_{h}$, associated with (1.2), as follows:

Find $\left(\mathbf{y}_{d}, p_{h}\right) \in \mathbf{X}_{h} \times M_{h}$ satisfying

$a\left(\mathbf{y}_{h}, \mathbf{w}_{h}\right)+b\left(\mathbf{y}_{h}, \mathbf{y}_{h}, \mathbf{w}_{h}\right)-\left(p_{h}, \operatorname{div} \mathbf{w}_{h}\right)=\left(\mathbf{f}+\mathcal{C} \mathbf{u}, \mathbf{w}_{h}\right) \forall \mathbf{w}_{h} \in \mathbf{X}_{h}$,

$\left(\lambda_{h}, \operatorname{div} \mathbf{y}_{h}\right)=0 \forall \lambda_{h} \in M_{h}$. 
For a given $\mathbf{u} \in L^{2}\left(\omega ; \mathbb{R}^{m}\right)$, this equation does not necessarily have a unique solution $\mathbf{y}_{h}$. Our main objective in this section is to show that there exist $\rho_{1}>0$ and $\rho_{2}>0$ independent of $h$, such that, for all $\mathbf{u} \in B_{\rho_{2}}(\overline{\mathbf{u}}),(4.1)$ admits a unique solution in $B_{\rho_{1}}(\overline{\mathbf{y}}) \times B_{\rho_{1}}(\bar{p})$. Let $T$ be the bounded linear operator from $\mathbf{H}^{-1}(\Omega)$ to $\mathbf{V}_{0}^{1}(\Omega) \times L_{0}^{2}(\Omega)$ defined by $T \mathbf{g}=(\mathbf{z}, q)$, where $(\mathbf{z}, q)$ is the solution of

$$
-\nu \Delta \mathbf{z}+\nabla q=\mathbf{g} \text { in } \Omega, \operatorname{div} \mathbf{z}=0 \text { in } \Omega, \mathbf{z}=0 \text { on } \Gamma .
$$

Let $\mathcal{F}$ be the nonlinear mapping from $L^{2}\left(\omega ; \mathbb{R}^{m}\right) \times \mathbf{H}_{0}^{1}(\Omega) \times L_{0}^{2}(\Omega)$ into $\mathbf{H}_{0}^{1}(\Omega) \times L_{0}^{2}(\Omega)$ defined by

$$
\mathcal{F}(\mathbf{u}, \mathbf{y}, p)=(\mathbf{y}, p)+T[B(\mathbf{y})-(\mathbf{f}+\mathcal{C} \mathbf{u})] .
$$

Notice that $\mathcal{F}(\mathbf{u}, \mathbf{y}, p)=0$ if and only if $A \mathbf{y}+B(\mathbf{y})=\mathbf{f}+\mathcal{C} \mathbf{u}$ in $\mathbf{V}^{-1}(\Omega)$ and $p \in L_{0}^{2}(\Omega)$ satisfies $\nabla p=(I-P)(\mathbf{f}+\mathcal{C} \mathbf{u}+\nu \Delta \mathbf{y}-(\mathbf{y} \cdot \nabla) \mathbf{y})$. The operator $\partial_{(\mathbf{y}, p)} \mathcal{F}(\mathbf{u}, \mathbf{y}, p)$ belongs to $\mathcal{L}\left(\mathbf{H}_{0}^{1}(\Omega) \times L_{0}^{2}(\Omega)\right)$ and is defined by

$$
\partial_{(\mathbf{y}, p)} \mathcal{F}(\mathbf{u}, \mathbf{y}, p)(\mathbf{z}, q)=(\mathbf{z}, q)+T\left[B^{\prime}(\mathbf{y}) \mathbf{z}\right] .
$$

Observe that $\partial_{(\mathbf{y}, p)} \mathcal{F}(\mathbf{u}, \mathbf{y}, p)$ does not depend on $\mathbf{u} \in L^{2}\left(\omega ; \mathbb{R}^{m}\right)$ and $p \in L_{0}^{2}(\Omega)$.

Lemma 4.2. Let $(\overline{\mathbf{u}}, \overline{\mathbf{y}}) \in L^{2}\left(\omega ; \mathbb{R}^{m}\right) \times \mathbf{V}_{0}^{1}(\Omega)$ be a solution of $(1.2)$, with associated pressure $\bar{p}$. Then $(\overline{\mathbf{u}}, \overline{\mathbf{y}})$ is a nonsingular solution if and only if $\partial_{(\mathbf{y}, p)} \mathcal{F}(\overline{\mathbf{u}}, \overline{\mathbf{y}}, \bar{p})$ is an automorphism in $\mathbf{H}_{0}^{1}(\Omega) \times L_{0}^{2}(\Omega)$.

Proof. Let us assume that $(\overline{\mathbf{u}}, \overline{\mathbf{y}}) \in L^{2}\left(\omega ; \mathbb{R}^{m}\right) \times \mathbf{V}_{0}^{1}(\Omega)$ is a nonsingular solution of (1.2). Let $(\hat{\mathbf{y}}, \hat{p})$ be in $\mathbf{H}_{0}^{1}(\Omega) \times L_{0}^{2}(\Omega)$. We have to check that there exists a unique pair $(\mathbf{y}, p) \in \mathbf{H}_{0}^{1}(\Omega) \times L_{0}^{2}(\Omega)$ such that $(\mathbf{y}, p)+T\left[B^{\prime}(\overline{\mathbf{y}}) \mathbf{y}\right]=(\hat{\mathbf{y}}, \hat{p})$. Let $\left(\mathbf{y}_{0}, p_{0}\right) \in \mathbf{V}_{0}^{1}(\Omega) \times L_{0}^{2}(\Omega)$ be the unique solution of the equation

$$
-\nu \Delta \mathbf{y}_{0}+B^{\prime}(\overline{\mathbf{y}}) \mathbf{y}_{0}+\nabla p_{0}=-B^{\prime}(\overline{\mathbf{y}}) \hat{\mathbf{y}} \text { in } \Omega, \operatorname{div} \mathbf{y}_{0}=0 \text { in } \Omega, \mathbf{y}_{0}=0 \text { on } \Gamma .
$$

Set $\mathbf{y}=\mathbf{y}_{0}+\hat{\mathbf{y}}$ and $p=p_{0}+\hat{p}$. The equality $(\mathbf{y}, p)+T\left[B^{\prime}(\overline{\mathbf{y}})(\mathbf{y})\right]=(\hat{\mathbf{y}}, \hat{p})$, i.e., $T\left[-B^{\prime}(\overline{\mathbf{y}}) \mathbf{y}_{0}-B^{\prime}(\overline{\mathbf{y}}) \hat{\mathbf{y}}\right]=\left(\mathbf{y}_{0}, p_{0}\right)$, follows from the definition of $T$ and of $\left(\mathbf{y}_{0}, p_{0}\right)$. So we have proved the surjectivity of $\partial_{(\mathbf{y}, p)} \mathcal{F}(\overline{\mathbf{u}}, \overline{\mathbf{y}}, \bar{p})$. For the injectivity let us assume that $(\mathbf{y}, p)+T\left[B^{\prime}(\overline{\mathbf{y}}) \mathbf{y}\right]=(0,0)$. This implies that $A \mathbf{y}+B^{\prime}(\overline{\mathbf{y}}) \mathbf{y}=0$ in $\mathbf{V}^{-1}(\Omega)$; then $\mathbf{y}=0$ and therefore $p=0$ too.

Conversely, let us assume that $\partial_{(\mathbf{y}, p)} \mathcal{F}(\overline{\mathbf{u}}, \overline{\mathbf{y}}, \bar{p})$ is an automorphism in $\mathbf{H}_{0}^{1}(\Omega) \times$ $L_{0}^{2}(\Omega)$. Let $\mathbf{g} \in \mathbf{V}^{-1}(\Omega)$. Let $(\hat{\mathbf{y}}, \hat{p}) \in \mathbf{V}_{0}^{1}(\Omega) \times L_{0}^{2}(\Omega)$ be the solution of the equation

$$
-\nu \Delta \hat{\mathbf{y}}+\nabla \hat{p}=\mathbf{g} .
$$

Let $(\mathbf{y}, p) \in \mathbf{H}_{0}^{1}(\Omega) \times L_{0}^{2}(\Omega)$ be the solution of the equation

$$
\partial_{(\mathbf{y}, p)} \mathcal{F}(\overline{\mathbf{u}}, \overline{\mathbf{y}}, \bar{p})(\mathbf{y}, p)=(\hat{\mathbf{y}}, \hat{p}) .
$$

It is easy to check that $\mathbf{y} \in \mathbf{V}_{0}^{1}(\Omega)$ is the unique solution of $A \mathbf{y}+B^{\prime}(\overline{\mathbf{y}}) \mathbf{y}=\mathbf{g}$.

Let $T_{h}$ be the bounded linear operator from $\mathbf{H}^{-1}(\Omega)$ to $\mathbf{X}_{h} \times M_{h}$ defined by $T_{h} \mathbf{g}=\left(\mathbf{z}_{h}, q_{h}\right)$, where $\left(\mathbf{z}_{h}, q_{h}\right) \in \mathbf{X}_{h} \times M_{h}$ is the solution of

$$
\begin{aligned}
& a\left(\mathbf{z}_{h}, \mathbf{w}_{h}\right)-\left(q_{h}, \operatorname{div} \mathbf{w}_{h}\right)=\left(\mathbf{g}, \mathbf{w}_{h}\right) \forall \mathbf{w}_{h} \in \mathbf{X}_{h}, \\
& \left(\lambda_{h}, \operatorname{div} \mathbf{z}_{h}\right)=0 \forall \lambda_{h} \in M_{h} .
\end{aligned}
$$

Let $\mathcal{F}_{h}$ be the nonlinear mapping from $L^{2}\left(\omega ; \mathbb{R}^{m}\right) \times \mathbf{H}_{0}^{1}(\Omega) \times L_{0}^{2}(\Omega)$ into $\mathbf{H}_{0}^{1}(\Omega) \times L_{0}^{2}(\Omega)$ defined by

$$
\mathcal{F}_{h}(\mathbf{u}, \mathbf{y}, p)=(\mathbf{y}, p)+T_{h}[B(\mathbf{y})-(\mathbf{f}+\mathcal{C} \mathbf{u})]
$$


Remark 4.3. Notice that if $\mathcal{F}_{h}(\mathbf{u}, \mathbf{y}, p)=0$, then $(\mathbf{y}, p)$ belongs to $\mathbf{X}_{h} \times M_{h}$ and is a solution of (4.1). Conversely if $(\mathbf{y}, p) \in \mathbf{X}_{h} \times M_{h}$ is a solution of (4.1), then $\mathcal{F}_{h}(\mathbf{u}, \mathbf{y}, p)=0$.

Now we want to prove that if $\overline{\mathbf{y}}$ is nonsingular and if $\|\mathbf{y}-\overline{\mathbf{y}}\|_{\mathbf{H}_{0}^{1}(\Omega)}$ is small enough, then $\partial_{(\mathbf{y}, p)} \mathcal{F}_{h}(\mathbf{u}, \mathbf{y}, p)$ is an automorphism in $\mathbf{H}_{0}^{1}(\Omega) \times L_{0}^{2}(\Omega)$. For that we make the following additional and usual assumptions concerning the approximation results for the Stokes problem.

(S1) $\lim _{h \rightarrow 0}\left\|\left(T-T_{h}\right) \mathbf{g}\right\|_{\mathbf{H}_{0}^{1}(\Omega) \times L_{0}^{2}(\Omega)}=0 \forall \mathbf{g} \in \mathbf{H}^{-1}(\Omega)$.

(S2) $\left\|\left(T-T_{h}\right) \mathbf{g}\right\|_{\mathbf{H}_{0}^{1}(\Omega) \times L_{0}^{2}(\Omega)} \leq C h\|\mathbf{g}\|_{\mathbf{L}^{2}(\Omega)} \forall \mathbf{g} \in \mathbf{L}^{2}(\Omega)$.

Before proving the desired property of $\partial_{(\mathbf{y}, p)} \mathcal{F}_{h}(\mathbf{u}, \mathbf{y}, p)$, we establish several lemmas.

Lemma 4.4. There exists $C>0$ independent of $h$ such that

$$
\left\|T_{h}\right\|_{\mathcal{L}\left(\mathbf{H}^{-1}(\Omega), \mathbf{H}_{0}^{1}(\Omega) \times L_{0}^{2}(\Omega)\right)} \leq C .
$$

Proof. We want to estimate $\sup \left\{\left\|T_{h} \mathbf{g}\right\|_{\mathbf{H}_{0}^{1}(\Omega) \times L_{0}^{2}(\Omega)} \mid\|\mathbf{g}\|_{\mathbf{H}^{-1}(\Omega)} \leq 1\right\}$. Recall that $T_{h} \mathbf{g}$ is the solution $\left(\mathbf{z}_{h}, q_{h}\right)$ to the discrete Stokes problem

$$
\begin{aligned}
& a\left(\mathbf{z}_{h}, \mathbf{w}_{h}\right)-\left(q_{h}, \operatorname{div} \mathbf{w}_{h}\right)=\left(g, \mathbf{w}_{h}\right) \forall \mathbf{w}_{h} \in \mathbf{X}_{h}, \\
& \left(\lambda_{h}, \operatorname{div} \mathbf{z}_{h}\right)=0 \forall \lambda_{h} \in M_{h} .
\end{aligned}
$$

Taking $\mathbf{w}_{h}=\mathbf{z}_{h}$, we obtain $\left\|\mathbf{z}_{h}\right\|_{\mathbf{H}_{0}^{1}(\Omega)} \leq C\|\mathbf{g}\|_{\mathbf{H}^{-1}(\Omega)}$. The estimate for the pressure $q_{h}$ follows from inf-sup condition (H3). Indeed if we take $\mathbf{w}_{h}$ such that $\left(q_{h}, \operatorname{div} \mathbf{w}_{h}\right)=$ $\left\|q_{h}\right\|_{L_{0}^{2}(\Omega)}^{2}$ and $\left\|\mathbf{w}_{h}\right\|_{\mathbf{H}_{0}^{1}(\Omega)} \leq C\left\|q_{h}\right\|_{L_{0}^{2}(\Omega)}$, it is clear that $\left\|q_{h}\right\|_{L_{0}^{2}(\Omega)} \leq C\|g\|_{\mathbf{H}^{-1}(\Omega)}$.

We will need the following standard result.

Lemma 4.5. Let $X$ be a Banach space, $A \in \mathcal{L}(X)$ invertible and $B \in \mathcal{L}(X)$. If $\|A-B\|_{\mathcal{L}(X)}<1 /\left\|A^{-1}\right\|_{\mathcal{L}(X)}$, then $B$ is invertible. If $\|A-B\|_{\mathcal{L}(X)}<1 /\left(2\left\|A^{-1}\right\|_{\mathcal{L}(X)}\right)$, then $\left\|B^{-1}\right\|_{\mathcal{L}(X)} \leq 2\left\|A^{-1}\right\|_{\mathcal{L}(X)}$.

Proof. $A^{-1} B=I-A^{-1}(A-B)$. Since $\left\|A^{-1}(A-B)\right\| \leq\left\|A^{-1}\right\|\|A-B\|<1$, we have that $A^{-1} B$ is invertible and so is $B$.

$B^{-1} A=\left(I-A^{-1}(A-B)\right)^{-1}=\sum_{k=1}^{\infty}\left(A^{-1}(A-B)\right)^{k}$. So $\left\|B^{-1}\right\| \leq\left\|A^{-1}\right\| /(1-$ $\left.\left\|A^{-1}(A-B)\right\|\right) \leq 2\left\|A^{-1}\right\|$.

Lemma 4.6. Let $\overline{\mathbf{y}} \in \mathbf{V}_{0}^{1}(\Omega)$ be a nonsingular solution of (2.2). Then for every $\varepsilon>0$ there exist $h_{\varepsilon}>0$ and $\rho_{\varepsilon}>0$ such that

$$
\left\|T\left[B^{\prime}(\overline{\mathbf{y}})\right]-T_{h}\left[B^{\prime}(\mathbf{y})\right]\right\|_{\mathcal{L}\left(\mathbf{H}_{0}^{1}(\Omega), \mathbf{H}_{0}^{1}(\Omega) \times L_{0}^{2}(\Omega)\right)}<\varepsilon
$$

for all $0<h<h_{\varepsilon}$ and all $\mathbf{y} \in B_{\rho_{\varepsilon}}(\overline{\mathbf{y}})$.

Proof. With classical calculations we can write

$$
\begin{aligned}
& \left\|T\left[B^{\prime}(\overline{\mathbf{y}})\right]-T_{h}\left[B^{\prime}(\mathbf{y})\right]\right\|_{\mathcal{L}\left(\mathbf{H}_{0}^{1}(\Omega), \mathbf{H}_{0}^{1}(\Omega) \times L_{0}^{2}(\Omega)\right)} \\
& \leq\left\|\left(T-T_{h}\right)\left[B^{\prime}(\overline{\mathbf{y}})\right]\right\|_{\mathcal{L}\left(\mathbf{H}_{0}^{1}(\Omega), \mathbf{H}_{0}^{1}(\Omega) \times L_{0}^{2}(\Omega)\right)}+\left\|T_{h}\left[B^{\prime}(\overline{\mathbf{y}})-B^{\prime}(\mathbf{y})\right]\right\|_{\mathcal{L}\left(\mathbf{H}_{0}^{1}(\Omega), \mathbf{H}_{0}^{1}(\Omega) \times L_{0}^{2}(\Omega)\right)} \\
& \leq \sup _{\|\mathbf{z}\|_{\mathbf{H}_{0}^{1}(\Omega)} \leq 1}\left\|\left(T-T_{h}\right)\left[B^{\prime}(\overline{\mathbf{y}}) \mathbf{z}\right]\right\|_{\mathbf{H}_{0}^{1}(\Omega) \times L_{0}^{2}(\Omega)} \\
& +\sup _{\|\mathbf{z}\|_{\mathbf{H}_{0}^{1}(\Omega)} \leq 1}\left\|T_{h}\left[\left(B^{\prime}(\overline{\mathbf{y}})-B^{\prime}(\mathbf{y})\right) \mathbf{z}\right]\right\|_{\mathbf{H}_{0}^{1}(\Omega) \times L_{0}^{2}(\Omega)} .
\end{aligned}
$$

Since $\overline{\mathbf{y}} \in \mathbf{H}^{2}(\Omega), B^{\prime}(\overline{\mathbf{y}}) \mathbf{z}$ belongs to $\mathbf{L}^{2}(\Omega)$, and due to assumption (S2) we have

$$
\begin{aligned}
& \sup _{\|\mathbf{z}\|_{\mathbf{H}_{0}^{1}(\Omega)} \leq 1}\left\|\left(T-T_{h}\right)\left[B^{\prime}(\overline{\mathbf{y}}) \mathbf{z}\right]\right\|_{\mathbf{H}_{0}^{1}(\Omega) \times L_{0}^{2}(\Omega)} \\
& \leq C h \sup _{\|\mathbf{z}\|_{\mathbf{H}_{0}^{1}(\Omega)} \leq 1}\left\|B^{\prime}(\overline{\mathbf{y}}) \mathbf{z}\right\|_{\mathbf{L}^{2}(\Omega)} \leq C h\|\overline{\mathbf{y}}\|_{\mathbf{H}^{2}(\Omega)} .
\end{aligned}
$$


On the other hand, using Lemma 4.4 we have

$$
\begin{aligned}
& \sup _{\|\mathbf{z}\|_{\mathbf{H}_{0}^{1}(\Omega)} \leq 1}\left\|T_{h}\left[\left(B^{\prime}(\overline{\mathbf{y}})-B^{\prime}(\mathbf{y})\right) \mathbf{z}\right]\right\|_{\mathbf{H}_{0}^{1}(\Omega) \times L_{0}^{2}(\Omega)} \\
& \leq\left\|T_{h}\right\|_{\mathcal{L}\left(\mathbf{H}^{-1}(\Omega), \mathbf{H}_{0}^{1}(\Omega) \times L_{0}^{2}(\Omega)\right)} \sup _{\|\mathbf{z}\|_{\mathbf{H}_{0}^{1}(\Omega)} \leq 1}\left\|\left(B^{\prime}(\overline{\mathbf{y}})-B^{\prime}(\mathbf{y})\right) \mathbf{z}\right\|_{\mathbf{H}^{-1}(\Omega)} \\
& \leq C\|\overline{\mathbf{y}}-\mathbf{y}\|_{\mathbf{H}_{0}^{1}(\Omega)} .
\end{aligned}
$$

Taking $h_{\varepsilon}$ and $\rho_{\varepsilon}$ small enough, we obtain the desired result.

THEOREM 4.7. Let $(\overline{\mathbf{u}}, \overline{\mathbf{y}}) \in L^{2}\left(\omega ; \mathbb{R}^{m}\right) \times \mathbf{V}_{0}^{1}(\Omega)$ be a nonsingular solution of (2.2) and $\bar{p}$ the associated pressure. There exist $h_{0}>0$ and $\rho_{0}>0$ such that for all $0<$ $h<h_{0}$ and all $\mathbf{y} \in B_{\rho_{0}}(\overline{\mathbf{y}}), \partial_{(\mathbf{y}, p)} \mathcal{F}_{h}(\mathbf{u}, \mathbf{y}, p)$ is an automorphism in $\mathbf{H}_{0}^{1}(\Omega) \times L_{0}^{2}(\Omega)$, and

$$
\left\|\partial_{(\mathbf{y}, p)} \mathcal{F}_{h}(\mathbf{u}, \mathbf{y}, p)^{-1}\right\|_{\mathcal{L}\left(\mathbf{H}_{0}^{1}(\Omega) \times L_{0}^{2}(\Omega)\right)} \leq 2\left\|\partial_{(\mathbf{y}, p)} \mathcal{F}(\overline{\mathbf{u}}, \overline{\mathbf{y}}, \bar{p})^{-1}\right\|_{\mathcal{L}\left(\mathbf{H}_{0}^{1}(\Omega) \times L_{0}^{2}(\Omega)\right)} .
$$

Proof. The proof is a straightforward consequence of the previous lemmas. Take

$$
\varepsilon=\frac{1}{2\left\|\partial_{(\mathbf{y}, p)} \mathcal{F}(\overline{\mathbf{u}}, \overline{\mathbf{y}}, \bar{p})^{-1}\right\|_{\mathcal{L}\left(\mathbf{H}_{0}^{1}(\Omega) \times L_{0}^{2}(\Omega)\right)}},
$$

and set $\left(h_{0}, \rho_{0}\right)=\left(h_{\varepsilon}, \rho_{\varepsilon}\right)$, where $\left(h_{\varepsilon}, \rho_{\varepsilon}\right)$ is the pair corresponding to $\varepsilon$ and defined in Lemma 4.6. For every $0<h<h_{0}$ and all $\mathbf{y} \in B_{\rho_{0}}(\overline{\mathbf{y}})$, we have

$$
\begin{gathered}
\left\|\partial_{(\mathbf{y}, p)} \mathcal{F}(\overline{\mathbf{u}}, \overline{\mathbf{y}}, \bar{p})-\partial_{(\mathbf{y}, p)} \mathcal{F}_{h}(\mathbf{u}, \mathbf{y}, p)\right\|_{\mathcal{L}\left(\mathbf{H}_{0}^{1}(\Omega) \times L_{0}^{2}(\Omega)\right)}= \\
\left\|T\left[B^{\prime}(\overline{\mathbf{y}})\right]-T_{h}\left[B^{\prime}(\mathbf{y})\right]\right\|_{\mathcal{L}\left(\mathbf{H}_{0}^{1}(\Omega), \mathbf{H}_{0}^{1}(\Omega) \times L_{0}^{2}(\Omega)\right)}<\varepsilon,
\end{gathered}
$$

and the result follows from Lemma 4.5.

TheOrem 4.8. Let $(\overline{\mathbf{u}}, \overline{\mathbf{y}})$ be as in Theorem 4.7 ; then there exist $\rho_{1}>0, \rho_{2}>0$, and $h_{1}>0$ such that for all $0<h<h_{1}$ and $\mathbf{u} \in B_{\rho_{2}}(\overline{\mathbf{u}})$, the equation $\mathcal{F}_{h}\left(\mathbf{u}, \mathbf{y}_{h}, p_{h}\right)=$ 0 admits a unique solution in $B_{\rho_{1}}(\overline{\mathbf{y}}) \times B_{\rho_{1}}(\bar{p})$.

Proof. Let $\rho_{0}$ and $h_{0}$ be the positive constants given by Theorem 4.7. For $\rho \leq$ $\rho_{0}, h \leq h_{0}$, and $\mathbf{u} \in B_{\rho^{2}}(\overline{\mathbf{u}})$, we define the mapping $\Psi_{\mathbf{u}}$ from $B_{\rho}(\overline{\mathbf{y}}) \times B_{\rho}(\bar{p})$ into $\mathbf{H}_{0}^{1}(\Omega) \times L_{0}^{2}(\Omega)$ by

$$
\Psi_{\mathbf{u}}(\mathbf{y}, p)=(\mathbf{y}, p)-\left[\partial_{(\mathbf{y}, p)} \mathcal{F}_{h}(\overline{\mathbf{u}}, \overline{\mathbf{y}}, \bar{p})\right]^{-1} \mathcal{F}_{h}(\mathbf{u}, \mathbf{y}, p) .
$$

It is clear that any fixed point of $\Psi_{\mathbf{u}}$ is a solution of $\mathcal{F}_{h}(\mathbf{u}, \mathbf{y}, p)=0$. Let us show that $\Psi_{\mathbf{u}}$ is a strict contraction if $\rho$ is small enough.

(i) First, we show that $\Psi_{\mathbf{u}}$ is a mapping from $B_{\rho}(\overline{\mathbf{y}}) \times B_{\rho}(\bar{p})$ into itself. With the identity $\mathcal{F}(\overline{\mathbf{u}}, \overline{\mathbf{y}}, \bar{p})=0$, and a Taylor formula we obtain

$$
\begin{aligned}
& \left\|\Psi_{\mathbf{u}}(\mathbf{y}, p)-(\overline{\mathbf{y}}, \bar{p})\right\|_{\mathbf{H}_{0}^{1}(\Omega) \times L_{0}^{2}(\Omega)} \\
& =\|\left[\partial_{(\mathbf{y}, p)} \mathcal{F}_{h}(\overline{\mathbf{u}}, \overline{\mathbf{y}}, \bar{p})\right]^{-1}\left\{\partial_{(\mathbf{y}, p)} \mathcal{F}_{h}(\overline{\mathbf{u}}, \overline{\mathbf{y}}, \bar{p})(\mathbf{y}-\overline{\mathbf{y}}, p-\bar{p})\right. \\
& \left.\quad+\left[-\mathcal{F}_{h}(\mathbf{u}, \mathbf{y}, p)+\mathcal{F}_{h}(\mathbf{u}, \overline{\mathbf{y}}, \bar{p})\right]+\left[-\mathcal{F}_{h}(\mathbf{u}, \overline{\mathbf{y}}, \bar{p})+\mathcal{F}(\overline{\mathbf{u}}, \overline{\mathbf{y}}, \bar{p})\right]\right\} \|_{\mathbf{H}_{0}^{1}(\Omega) \times L_{0}^{2}(\Omega)} \\
& \leq C \| \partial_{(\mathbf{y}, p)} \mathcal{F}_{h}(\overline{\mathbf{u}}, \overline{\mathbf{y}}, \bar{p})(\mathbf{y}-\overline{\mathbf{y}}, p-\bar{p}) \\
& \quad-\int_{0}^{1} \partial_{(\mathbf{y}, p)} \mathcal{F}_{h}\left(\overline{\mathbf{u}}, \mathbf{y}_{\theta}, p_{\theta}\right)(\mathbf{y}-\overline{\mathbf{y}}, p-\bar{p}) d \theta \|_{\mathbf{H}_{0}^{1}(\Omega) \times L_{0}^{2}(\Omega)} \\
& \quad+C\left\|\mathcal{F}_{h}(\mathbf{u}, \overline{\mathbf{y}}, \bar{p})-\mathcal{F}(\overline{\mathbf{u}}, \overline{\mathbf{y}}, \bar{p})\right\|_{\mathbf{H}_{0}^{1}(\Omega) \times L_{0}^{2}(\Omega)} \\
& \leq C \int_{0}^{1}\left\|\partial_{(\mathbf{y}, p)} \mathcal{F}_{h}\left(\overline{\mathbf{u}}, \mathbf{y}_{\theta}, p_{\theta}\right)-\partial_{(\mathbf{y}, p)} \mathcal{F}_{h}(\overline{\mathbf{u}}, \overline{\mathbf{y}}, \bar{p})\right\|_{\mathcal{L}\left(\mathbf{H}_{0}^{1}(\Omega) \times L_{0}^{2}(\Omega)\right)} d \theta \\
& \quad \times\|(\mathbf{y}-\overline{\mathbf{y}}, p-\bar{p})\|_{\mathbf{H}_{0}^{1}(\Omega) \times L_{0}^{2}(\Omega)} \\
& \quad+C\left\|\left(T_{h}-T\right)[B(\overline{\mathbf{y}})-\mathbf{f}]\right\|_{\mathbf{H}_{0}^{1}(\Omega) \times L_{0}^{2}(\Omega)}+C\left\|\left(T-T_{h}\right)[\mathcal{C} \overline{\mathbf{u}}]\right\|_{\mathbf{H}_{0}^{1}(\Omega) \times L_{0}^{2}(\Omega)} \\
& \quad+C\left\|T_{h}[\mathcal{C}(\overline{\mathbf{u}}-\mathbf{u})]\right\|_{\mathbf{H}_{0}^{1}(\Omega) \times L_{0}^{2}(\Omega)}
\end{aligned}
$$


where $\left(\mathbf{y}_{\theta}, p_{\theta}\right)=(\overline{\mathbf{y}}+\theta(\mathbf{y}-\overline{\mathbf{y}}), \bar{p}+\theta(p-\bar{p}))$.

Let us estimate each of the terms. Using the definition of $\mathcal{F}_{h}$ and Lemma 4.4 we get

$$
\begin{aligned}
& \left\|\partial_{(\mathbf{y}, p)} \mathcal{F}_{h}(\overline{\mathbf{u}}, \overline{\mathbf{y}}+\theta(\mathbf{y}-\overline{\mathbf{y}}), \bar{p}+\theta(p-\bar{p}))-\partial_{(\mathbf{y}, p)} \mathcal{F}_{h}(\overline{\mathbf{u}}, \overline{\mathbf{y}}, \bar{p})\right\|_{\mathcal{L}\left(\mathbf{H}_{0}^{1}(\Omega) \times L_{0}^{2}(\Omega)\right)} \\
& \quad=\left\|T_{h}\left[B^{\prime}(\overline{\mathbf{y}}+\theta(\mathbf{y}-\overline{\mathbf{y}}))-B^{\prime}(\overline{\mathbf{y}})\right]\right\|_{\mathcal{L}\left(\mathbf{H}_{0}^{1}(\Omega) \times L_{0}^{2}(\Omega)\right)} \\
& \quad \leq C\left\|B^{\prime}(\mathbf{y}-\overline{\mathbf{y}})\right\|_{\mathcal{L}\left(\mathbf{H}_{0}^{1}(\Omega), \mathbf{H}^{-1}(\Omega)\right)} \leq C\|\mathbf{y}-\overline{\mathbf{y}}\|_{\mathbf{H}_{0}^{1}(\Omega)} .
\end{aligned}
$$

With assumption (S2) we have

$$
\left\|\left(T_{h}-T\right)[B(\overline{\mathbf{y}})-\mathbf{f}]\right\|_{\mathbf{H}_{0}^{1}(\Omega) \times L_{0}^{2}(\Omega)} \leq C h\left(\|\overline{\mathbf{y}}\|_{\mathbf{H}^{2}(\Omega)}+\|\mathbf{f}\|_{\mathbf{L}^{2}(\Omega)}\right),
$$

and

$$
\left\|\left(T-T_{h}\right)[\mathcal{C} \overline{\mathbf{u}}]\right\|_{\mathbf{H}_{0}^{1}(\Omega) \times L_{0}^{2}(\Omega)} \leq C h\|\overline{\mathbf{u}}\|_{L^{2}\left(\omega ; \mathbb{R}^{m}\right)} .
$$

Finally, from Lemma 4.4 it follows that

$$
\left\|T_{h}[\mathcal{C}(\overline{\mathbf{u}}-\mathbf{u})]\right\|_{\mathbf{H}_{0}^{1}(\Omega) \times L_{0}^{2}(\Omega)} \leq C\|\overline{\mathbf{u}}-\mathbf{u}\|_{L^{2}\left(\omega ; \mathbb{R}^{m}\right)} .
$$

Collecting these estimates all together, we have proved that there exists a constant $\hat{C}>0$ independent of $h$ and $\rho$ such that

$$
\left\|\Psi_{\mathbf{u}}(\mathbf{y}, p)-(\overline{\mathbf{y}}, \bar{p})\right\|_{\mathbf{H}_{0}^{1}(\Omega) \times L_{0}^{2}(\Omega)} \leq \hat{C}\left(h+\rho^{2}\right) .
$$

We choose $\hat{\rho}_{1} \leq \min \left\{\rho_{0}, 1 /(2 \hat{C})\right\}, \hat{\rho}_{2}=\hat{\rho}_{1}^{2}$, and $\hat{h}_{1}=\min \left\{h_{0}, \hat{\rho}_{1} /(2 \hat{C})\right\}$. It is clear that for all $0<h<\hat{h}_{1}$ and all $\mathbf{u} \in B_{\hat{\rho}_{2}}(\overline{\mathbf{u}}), \Psi_{\mathbf{u}}$ is a mapping from $B_{\hat{\rho}_{1}}(\overline{\mathbf{y}}) \times B_{\hat{\rho}_{1}}(\bar{p})$ into itself.

(ii) Now we look for conditions to have a strict contraction. Take $\left(\mathbf{y}_{1}, p_{1}\right),\left(\mathbf{y}_{2}, p_{2}\right) \in$ $B_{\hat{\rho}_{1}}(\overline{\mathbf{y}}) \times B_{\hat{\rho}_{1}}(\bar{p}), 0<h<\hat{h}_{1}$, and $\mathbf{u} \in B_{\hat{\rho}_{2}}(\overline{\mathbf{u}})$. Classical calculations lead to

$$
\begin{aligned}
& \left\|\Psi_{\mathbf{u}}\left(\mathbf{y}_{1}, p_{1}\right)-\Psi_{\mathbf{u}}\left(\mathbf{y}_{2}, p_{2}\right)\right\|_{\mathbf{H}_{0}^{1}(\Omega) \times L_{0}^{2}(\Omega)} \\
& =\|\left(\mathbf{y}_{1}-\mathbf{y}_{2}, p_{1}-p_{2}\right) \\
& -\left[\partial_{(\mathbf{y}, p)} \mathcal{F}_{h}(\overline{\mathbf{u}}, \overline{\mathbf{y}}, \bar{p})\right]^{-1}\left\{\mathcal{F}_{h}\left(\mathbf{u}, \mathbf{y}_{1}, p_{1}\right)-\mathcal{F}_{h}\left(\mathbf{u}, \mathbf{y}_{2}, p_{2}\right)\right\} \|_{\mathbf{H}_{0}^{1}(\Omega) \times L_{0}^{2}(\Omega)} \\
& =\|\left[\partial_{(\mathbf{y}, p)} \mathcal{F}_{h}(\overline{\mathbf{u}}, \overline{\mathbf{y}}, \bar{p})\right]^{-1}\left\{\partial_{(\mathbf{y}, p)} \mathcal{F}_{h}(\overline{\mathbf{u}}, \overline{\mathbf{y}}, \bar{p})\left(\mathbf{y}_{1}-\mathbf{y}_{2}, p_{1}-p_{2}\right)\right. \\
& \left.-\int_{0}^{1} \partial_{(\mathbf{y}, p)} \mathcal{F}_{h}\left(\overline{\mathbf{u}}, \mathbf{y}_{1}+\theta\left(\mathbf{y}_{2}-\mathbf{y}_{1}\right), p_{1}+\theta\left(p_{2}-p_{1}\right)\right)\left(\mathbf{y}_{1}-\mathbf{y}_{2}, p_{1}-p_{2}\right) d \theta\right\} \|_{\mathbf{H}_{0}^{1}(\Omega) \times L_{0}^{2}(\Omega)}
\end{aligned}
$$

The norm $\left\|\left[\partial_{(\mathbf{y}, p)} \mathcal{F}_{h}(\overline{\mathbf{u}}, \overline{\mathbf{y}}, \bar{p})\right]^{-1}\right\|_{\mathcal{L}\left(\mathbf{H}_{0}^{1}(\Omega) \times L_{0}^{2}(\Omega)\right)}$ can be estimated by a constant $C$ independent of $h$; see Theorem 4.7. To estimate the expression in brackets we can repeat the argument of inequalities (4.2), since $\mathbf{y}=\mathbf{y}_{1}+\theta\left(\mathbf{y}_{2}-\mathbf{y}_{1}\right) \in B_{\hat{\rho}_{1}}(\overline{\mathbf{y}})$. There then exists $\tilde{C}>0$ independent of $\hat{\rho}_{1}$ and $h$ such that

$$
\left\|\Psi_{\mathbf{u}}\left(\mathbf{y}_{1}, p_{1}\right)-\Psi_{\mathbf{u}}\left(\mathbf{y}_{2}, p_{2}\right)\right\|_{\mathbf{H}_{0}^{1}(\Omega) \times L_{0}^{2}(\Omega)} \leq \tilde{C} \hat{\rho}_{1}^{2} .
$$

Choosing $\rho_{1}=\min \left\{\rho_{0}, 1 /(2 \hat{C}), 1 / \sqrt{2 \tilde{C}}\right\}, \rho_{2}=\rho_{1}^{2}$, and $h_{1}=\min \left\{h_{0}, \rho_{1} /(2 \hat{C})\right\}$, we have established that, for all $0<h<h_{1}$ and all $\mathbf{u} \in B_{\rho_{2}}(\overline{\mathbf{u}}), \Psi_{\mathbf{u}}$ is a strict contraction in $B_{\rho_{1}}(\overline{\mathbf{y}}) \times B_{\rho_{1}}(\bar{p})$. 
Remark 4.9. We have proved that, for all $0<h<h_{1}$ and all $\mathbf{u} \in B_{\rho_{2}}(\overline{\mathbf{u}})$, the equation $\mathcal{F}_{h}\left(\mathbf{u}, \mathbf{y}_{h}, p_{h}\right)=0$ admits a unique solution $\left(\mathbf{y}_{h}(\mathbf{u}), p_{h}(\mathbf{u})\right)$ in $\left(B_{\rho_{1}}(\overline{\mathbf{y}}) \times\right.$ $\left.B_{\rho_{1}}(\bar{p})\right) \cap\left(\mathbf{X}_{h} \times M_{h}\right)$, and that $\partial_{(\mathbf{y}, p)} \mathcal{F}_{h}\left(\mathbf{u}, \mathbf{y}_{h}(\mathbf{u}), p_{h}(\mathbf{u})\right)$ is an automorphism in $\mathbf{H}_{0}^{1}(\Omega) \times L_{0}^{2}(\Omega)$. Therefore the mapping $G_{h}$ from $B_{\rho_{2}}(\overline{\mathbf{u}})$ into $\left(B_{\rho_{1}}(\overline{\mathbf{y}}) \times B_{\rho_{1}}(\bar{p})\right) \cap$ $\left(\mathbf{X}_{h} \times M_{h}\right)$ defined by $G_{h}(\mathbf{u})=\left(\mathbf{y}_{h}(\mathbf{u}), p_{h}(\mathbf{u})\right)$, obeys $\mathcal{F}_{h}\left(\mathbf{u}, G_{h}(\mathbf{u})\right)=0$, and the implicit function theorem implies that it is of class $C^{\infty}$ in the interior of the ball $B_{\rho_{2}}(\overline{\mathbf{u}})$. Notice that $G_{h}$ is not an approximation of $G$ because $G(\mathbf{u})=\mathbf{y}_{\mathbf{u}}$ is a velocity field, while $G_{h}(\mathbf{u})$ stands for a velocity field and a pressure.

4.2. Discretization of the control problem. For simplicity throughout the following we assume that $\omega$ is a polygonal domain. But we could consider a more general situation if we take into account the error we introduce by approximating $\omega$ by a polygonal domain.

For $h>0$, let $\mathcal{T}_{h}$ be a triangulation of $\omega$. Although the discretization of the control can be done independently of the discretization of the state equation, in practice, when we use the finite element method to approximate the state and adjoint state equation, the same family of triangulations is used. Some assumptions must be made on the family of triangulations in order to have the inverse estimate of assumption (H1)(d). We will suppose that the family is quasi-uniform (see, e.g., [9, p. 135]): In this case $h=\max _{T \in \mathcal{T}_{h}} \rho(T)$, where $\rho(T)$ is the diameter of the set $T$. We denote by $\sigma(T)$ the diameter of the largest ball contained in $T$. We assume there exist two positive constants $\rho$ and $\sigma$ such that

$$
\frac{\rho(T)}{\sigma(T)} \leq \sigma, \quad \frac{h}{\rho(T)} \leq \rho
$$

hold for all $T \in \mathcal{T}_{h}$ and all $0<h$.

In the following we would like to treat in the same way the cases when the control set is discretized and when it is not. We shall see that we obtain better estimates when the control set is not discretized. For that we set

$$
\begin{aligned}
U_{h} & =\left\{\mathbf{u} \in L^{2}\left(\omega ; \mathbb{R}^{m}\right)\left|u_{i}\right|_{T} \in P_{0}(T) \forall T \in \mathcal{T}_{h}\right\}, \\
U_{a d}^{h} & =\left\{\mathbf{u} \in U_{h} \mid \alpha_{i} \leq u_{i} \leq \beta_{i} \forall 1 \leq i \leq d\right\} .
\end{aligned}
$$

In the discrete control problem stated below, the case when the control set is not discretized corresponds to the choice $U_{a d, h}=U_{a d}$, while the case when the control set is discretized corresponds to $U_{a d, h}=U_{a d}^{h}$.

We can now define the discrete control problem associated with $(\mathrm{P})$ in the following way:

$$
\inf \left\{F(\mathbf{u}, \mathbf{y}) \mid(\mathbf{u}, \mathbf{y}, p) \in U_{a d, h} \times \mathbf{X}_{h} \times M_{h} \text { and }(\mathbf{u}, \mathbf{y}, p) \text { satisfies (4.1) }\right\} .
$$

Let us recall that $(\mathbf{u}, \mathbf{y}, p)$ satisfies (4.1) if and only if

$$
\mathcal{F}_{h}(\mathbf{u}, \mathbf{y}, p)=0 .
$$

Our aim is to study the existence of local minima of problems $\left(\mathrm{P}_{h}\right)$ which approximate the local minima of $(\mathrm{P})$. This can be proved for nonsingular local solutions of $(\mathrm{P})$. Let us start by proving some error estimates for the state equation. Given a nonsingular solution $(\overline{\mathbf{u}}, \overline{\mathbf{y}})$ of $(1.2)$, let $h_{1}>0$ and $\rho_{2}>0$ be given by Theorem 4.8. 
By using the function $G_{h}$ from $B_{\rho_{2}}(\overline{\mathbf{u}})$ into $\left(B_{\rho_{1}}(\overline{\mathbf{y}}) \times B_{\rho_{1}}(\bar{p})\right) \cap\left(\mathbf{X}_{h} \times M_{h}\right)$ introduced at the end of the previous section in Remark 4.9, we set $\left(\mathbf{y}_{\mathbf{u}}^{h}, p_{\mathbf{u}}^{h}\right)=G_{h}(\mathbf{u})=$ $\left(\mathbf{y}_{h}(\mathbf{u}), p_{h}(\mathbf{u})\right)$. Now we have the following result.

Lemma 4.10. Let $(\overline{\mathbf{u}}, \overline{\mathbf{y}})$ be as in Theorem 4.7. There exists a constant $C>0$ such that, for all $\mathbf{u}, \hat{\mathbf{u}} \in \bar{B}_{\rho_{2}}(\overline{\mathbf{u}})$, and $0<h<h_{1}$, the following estimates hold:

$$
\begin{aligned}
& \left\|\mathbf{y}_{\mathbf{u}}-\mathbf{y}_{\mathbf{u}}^{h}\right\|_{\mathbf{L}^{2}(\Omega)} \leq C h^{2}\left\|\mathbf{y}_{\mathbf{u}}\right\|_{\mathbf{H}^{2}(\Omega)}, \\
& \left\|\mathbf{y}_{\mathbf{u}}-\mathbf{y}_{\hat{\mathbf{u}}}^{h}\right\|_{\mathbf{H}_{0}^{1}(\Omega)}+\left\|p_{\mathbf{u}}-p_{\hat{\mathbf{u}}}^{h}\right\|_{L_{0}^{2}(\Omega)} \leq C\left(h+\|\mathbf{u}-\hat{\mathbf{u}}\|_{L^{2}\left(\omega ; \mathbb{R}^{m}\right)}\right) .
\end{aligned}
$$

Moreover, if $\mathbf{u}_{h} \in B_{\rho_{2}}(\overline{\mathbf{u}})$ and $\mathbf{u}_{h} \rightarrow \mathbf{u}$ weakly in $L^{2}\left(\omega ; \mathbb{R}^{m}\right)$, then $\mathbf{y}_{\mathbf{u}_{h}}^{h} \rightarrow \mathbf{y}_{\mathbf{u}}$ in $C\left(\bar{\Omega} ; \mathbb{R}^{d}\right)$.

Proof. (i) The estimate (4.4) directly follows from usual estimates for the approximation of the Navier-Stokes equations by a finite element method. See, for instance, Girault-Raviart [12, Theorem IV.4.2].

(ii) To prove (4.5), let us write

$$
\begin{array}{r}
\left\|\mathbf{y}_{\mathbf{u}}-\mathbf{y}_{\hat{\mathbf{u}}}^{h}\right\|_{\mathbf{H}_{0}^{1}(\Omega)} \leq\left\|\mathbf{y}_{\mathbf{u}}-\mathbf{y}_{\mathbf{u}}^{h}\right\|_{\mathbf{H}_{0}^{1}(\Omega)}+\left\|\mathbf{y}_{\mathbf{u}}^{h}-\mathbf{y}_{\hat{\mathbf{u}}}^{h}\right\|_{\mathbf{H}_{0}^{1}(\Omega)}, \\
\left\|p_{\mathbf{u}}-p_{\hat{\mathbf{u}}}^{h}\right\|_{L_{0}^{2}(\Omega)} \leq\left\|p_{\mathbf{u}}-p_{\mathbf{u}}^{h}\right\|_{L_{0}^{2}(\Omega)}+\left\|p_{\mathbf{u}}^{h}-p_{\hat{\mathbf{u}}}^{h}\right\|_{L_{0}^{2}(\Omega)} .
\end{array}
$$

Usual finite element estimates [12, estimate (4.7)] give us

$$
\left\|\mathbf{y}_{\mathbf{u}}-\mathbf{y}_{\mathbf{u}}^{h}\right\|_{\mathbf{H}_{0}^{1}(\Omega)}+\left\|p_{\mathbf{u}}-p_{\mathbf{u}}^{h}\right\|_{L_{0}^{2}(\Omega)} \leq C h .
$$

If $\mathbf{u}$ belongs to the interior of $B_{\rho_{2}}(\overline{\mathbf{u}})$, from the definition of $G_{h}$ it follows that

$$
G_{h}^{\prime}(\mathbf{u}) \mathbf{v}=-\left[\partial_{(\mathbf{y}, p)} \mathcal{F}_{h}\left(\mathbf{u}, \mathbf{y}_{\mathbf{u}}^{h}, p_{\mathbf{u}}^{h}\right)\right]^{-1} T_{h}[\mathcal{C} \mathbf{v}] .
$$

Hence, with Lemma 4.4 and Theorem 4.7 we obtain

$$
\begin{aligned}
& \left\|G_{h}(\mathbf{u})-G_{h}(\hat{\mathbf{u}})\right\|_{\mathbf{H}_{0}^{1}(\Omega) \times L_{0}^{2}(\Omega)} \\
& =\left\|\int_{0}^{1}\left[\partial_{(\mathbf{y}, p)} \mathcal{F}_{h}\left(\mathbf{u}_{\theta}, \mathbf{y}_{\mathbf{u}_{\theta}}^{h}, p_{\mathbf{u}_{\theta}}^{h}\right)\right]^{-1} T_{h}[\mathcal{C}(\mathbf{u}-\hat{\mathbf{u}})]\right\|_{\mathbf{H}_{0}^{1}(\Omega) \times L_{0}^{2}(\Omega)} \\
& \leq C\|\mathbf{u}-\hat{\mathbf{u}}\|_{L^{2}\left(\omega ; \mathbb{R}^{m}\right)},
\end{aligned}
$$

where $\mathbf{u}_{\theta}=\hat{\mathbf{u}}+\theta(\mathbf{u}-\hat{\mathbf{u}})$. Collecting the previous estimates, the proof of (4.5) is complete.

(iii) Let $\left(\mathbf{u}_{h}\right)_{h}$ be a sequence in $B_{\rho_{2}}(\overline{\mathbf{u}}) \cap U_{a d}$, weakly converging to $\mathbf{u}$ in $L^{2}\left(\omega ; \mathbb{R}^{m}\right)$. Due to Theorem 2.2, $\mathbf{y}_{\mathbf{u}}$ belongs to $\mathbf{W}^{2, \bar{r}}(\Omega)$ and $\left\{\mathbf{y}_{\mathbf{u}_{h}}\right\}_{h}$ is bounded in $\mathbf{W}^{2, \bar{r}}(\Omega)$. Thus it converges to $\mathbf{y}_{\mathbf{u}}$ in $\mathbf{L}^{p}(\Omega)$ for all $2 \leq p<\infty$, and the sequence $\left\{\mathbf{y}_{\mathbf{u}_{h}} \otimes \mathbf{y}_{\mathbf{u}_{h}}\right\}_{h}$ converges to $\mathbf{y}_{\mathbf{u}} \otimes \mathbf{y}_{\mathbf{u}}$ in $\left(\mathbf{L}^{p}(\Omega)\right)^{d}$ for all $2 \leq p<\infty$. The function $\mathbf{y}_{\mathbf{u}_{h}}-\mathbf{y}_{\mathbf{u}}$ satisfies the equation

$$
A\left(\mathbf{y}_{\mathbf{u}_{h}}-\mathbf{y}_{\mathbf{u}}\right)=\operatorname{div}\left(\left(\mathbf{y}_{\mathbf{u}} \otimes \mathbf{y}_{\mathbf{u}_{h}}\right)-\left(\mathbf{y}_{\mathbf{u}_{h}} \otimes \mathbf{y}_{\mathbf{u}}\right)\right)+\mathcal{C}\left(\mathbf{u}_{h}-\mathbf{u}\right) \quad \text { in } \mathbf{V}^{-1}(\Omega) .
$$

Let $p$ satisfy $d<p<6$. From classical estimates for the Stokes equations it follows that

$$
\begin{aligned}
& \left\|\mathbf{y}_{\mathbf{u}_{h}}-\mathbf{y}_{\mathbf{u}}\right\|_{\mathbf{W}^{1, p}(\Omega)} \leq C\left\|\operatorname{div}\left(\left(\mathbf{y}_{\mathbf{u}} \otimes \mathbf{y}_{\mathbf{u}_{h}}\right)-\left(\mathbf{y}_{\mathbf{u}_{h}} \otimes \mathbf{y}_{\mathbf{u}}\right)\right)+\mathcal{C}\left(\mathbf{u}_{h}-\mathbf{u}\right)\right\|_{\mathbf{W}^{-1, p}(\Omega)} \\
& \leq C\left(\left\|\left(\mathbf{y}_{\mathbf{u}} \otimes \mathbf{y}_{\mathbf{u}_{h}}\right)-\left(\mathbf{y}_{\mathbf{u}_{h}} \otimes \mathbf{y}_{\mathbf{u}}\right)\right\|_{\mathbf{L}^{p}(\Omega)}+\left\|\mathcal{C}\left(\mathbf{u}_{h}-\mathbf{u}\right)\right\|_{\mathbf{W}^{-1, p}(\Omega)}\right) .
\end{aligned}
$$

Since $\mathbf{W}^{1, p}(\Omega) \hookrightarrow \mathbf{L}^{\infty}(\Omega)$, and $\mathbf{L}^{2}(\Omega)$ is compactly embedded in $\mathbf{W}^{-1, p}(\Omega)$ (because $p<6)$, it is clear that $\left\{\mathbf{y}_{\mathbf{u}_{h}}\right\}_{h}$ tends to $\mathbf{y}_{\mathbf{u}}$ in $\mathbf{L}^{\infty}(\Omega)$. 
We have

$$
\begin{aligned}
& \left\|\mathbf{y}_{\mathbf{u}_{h}}^{h}-\mathbf{y}_{\mathbf{u}}\right\|_{\mathbf{L}^{\infty}(\Omega)} \leq\left\|\mathbf{y}_{\mathbf{u}_{h}}-\mathbf{y}_{\mathbf{u}}\right\|_{\mathbf{L}^{\infty}(\Omega)}+\left\|\mathbf{y}_{\mathbf{u}_{h}}^{h}-\mathbf{y}_{\mathbf{u}_{h}}\right\|_{\mathbf{L}^{\infty}(\Omega)} \\
& \leq\left\|\mathbf{y}_{\mathbf{u}_{h}}-\mathbf{y}_{\mathbf{u}}\right\|_{\mathbf{L}^{\infty}(\Omega)}+\left\|\mathbf{y}_{\mathbf{u}_{h}}^{h}-r_{h} \mathbf{y}_{\mathbf{u}_{h}}\right\|_{\mathbf{L}^{\infty}(\Omega)}+\left\|r_{h} \mathbf{y}_{\mathbf{u}_{h}}-\mathbf{y}_{\mathbf{u}_{h}}\right\|_{\mathbf{L}^{\infty}(\Omega)}
\end{aligned}
$$

From $(\mathrm{H} 1)(\mathrm{c})$ and $(\mathrm{H} 1)(\mathrm{d})$ we deduce that

$$
\left\|\mathbf{y}_{\mathbf{u}_{h}}-r_{h} \mathbf{y}_{\mathbf{u}_{h}}\right\|_{\mathbf{L}^{\infty}(\Omega)} \leq C h^{2-d / 2}\left\|\mathbf{y}_{\mathbf{u}_{h}}\right\|_{\mathbf{H}^{2}(\Omega)}
$$

and

$$
\begin{aligned}
& \left\|r_{h} \mathbf{y}_{\mathbf{u}_{h}}-\mathbf{y}_{\mathbf{u}_{h}}^{h}\right\|_{\mathbf{L}^{\infty}(\Omega)} \leq C h^{-d / 2}\left\|r_{h} \mathbf{y}_{\mathbf{u}_{h}}-\mathbf{y}_{\mathbf{u}_{h}}^{h}\right\|_{\mathbf{L}^{2}(\Omega)} \\
& \leq C h^{-d / 2}\left\|r_{h} \mathbf{y}_{\mathbf{u}_{h}}-\mathbf{y}_{\mathbf{u}_{h}}\right\|_{\mathbf{L}^{2}(\Omega)}+C h^{-d / 2}\left\|\mathbf{y}_{\mathbf{u}_{h}}-\mathbf{y}_{\mathbf{u}_{h}}^{h}\right\|_{\mathbf{L}^{2}(\Omega)} .
\end{aligned}
$$

With (H1)(b) and (4.4) we have

$$
\begin{aligned}
& \left\|r_{h} \mathbf{y}_{\mathbf{u}_{h}}-\mathbf{y}_{\mathbf{u}_{h}}\right\|_{\mathbf{L}^{2}(\Omega)} \leq C h^{2}\left\|\mathbf{y}_{\mathbf{u}_{h}}\right\|_{\mathbf{H}^{2}(\Omega)}, \\
& \left\|\mathbf{y}_{\mathbf{u}_{h}}-\mathbf{y}_{\mathbf{u}_{h}}^{h}\right\|_{\mathbf{L}^{2}(\Omega)} \leq C h^{2}\left\|\mathbf{y}_{\mathbf{u}_{h}}\right\|_{\mathbf{H}^{2}(\Omega)}
\end{aligned}
$$

Collecting together these estimates and the previous convergence result we have proved that $\left\{\mathbf{y}_{\mathbf{u}_{h}}^{h}\right\}_{h}$ converges to $\mathbf{y}_{\mathbf{u}}$ in $\mathbf{L}^{\infty}(\Omega)$.

THEOREM 4.11. Let us assume that $(\mathrm{P})$ has a nonsingular local minimum $(\overline{\mathbf{u}}, \overline{\mathbf{y}})$. Then there exists $h_{2}>0$ such that, for all $0<h<h_{2},\left(\mathrm{P}_{h}\right)$ has at least one solution. If, furthermore, $(\overline{\mathbf{u}}, \overline{\mathbf{y}})$ is a strict local minimum of $(\mathrm{P})$, then $\left(\mathrm{P}_{h}\right)$ has a local minimum $\left(\overline{\mathbf{u}}_{h}, \overline{\mathbf{y}}_{h}\right)$ in a neighborhood of $(\overline{\mathbf{u}}, \overline{\mathbf{y}})$ for all $0<h<h_{2}$ and the following identities hold:

$$
\lim _{h \rightarrow 0} J_{h}\left(\overline{\mathbf{u}}_{h}\right)=J(\overline{\mathbf{u}}), \lim _{h \rightarrow 0}\left\|\overline{\mathbf{u}}-\overline{\mathbf{u}}_{h}\right\|_{\mathbf{L}^{2}(\omega)}=0, \text { and } \lim _{h \rightarrow 0}\left\|\overline{\mathbf{y}}-\overline{\mathbf{y}}_{h}\right\|_{\mathbf{H}_{0}^{1}(\Omega)}=0,
$$

where $J_{h}\left(\overline{\mathbf{u}}_{h}\right)=F\left(\overline{\mathbf{u}}_{h}, \overline{\mathbf{y}}_{h}\right)$.

Proof. Let us start by proving that the set of feasible pairs $(\mathbf{u}, \mathbf{y})$ for problem $\left(\mathrm{P}_{h}\right)$ is nonempty for $h$ small enough. We prove it only in the case when $U_{a d, h}=U_{a d}^{h}$. The case when $U_{a d, h}=U_{a d}$ is obvious.

Since $(\overline{\mathbf{u}}, \overline{\mathbf{y}})$ is a nonsingular local minimum, with the aid of Theorem 4.8 we derive the existence of $\rho \leq \rho_{2}$ such that

$$
J(\overline{\mathbf{u}}) \leq J(\mathbf{u}) \quad \forall \mathbf{u} \in U_{a d} \cap B_{\rho}(\overline{\mathbf{u}}) .
$$

Let us define $\Pi_{h} \overline{\mathbf{u}} \in U_{h}$ by

$$
\left.\Pi_{h} \overline{\mathbf{u}}\right|_{T}=\frac{1}{|T|} \int_{T} \overline{\mathbf{u}}(x) d x .
$$

It is clear that $\Pi_{h} \overline{\mathbf{u}} \in U_{a d, h}$. Let us prove that it belongs to $B_{\rho}(\overline{\mathbf{u}})$ if $h$ is small enough. Since $\overline{\mathbf{u}}$ is Lipschitz continuous (see Theorem 3.5), we can write

$$
\begin{aligned}
& \int_{\omega}\left(\Pi_{h} \bar{u}_{i}(s)-\bar{u}_{i}(s)\right)^{2} d s=\sum_{T \subset \omega} \int_{T}\left(\frac{1}{|T|} \int_{T} \bar{u}_{i}(x) d x-\bar{u}_{i}(s)\right)^{2} d s \\
& =\sum_{T \subset \omega} \int_{T}\left(\bar{u}_{i}\left(x_{T}\right)-\bar{u}_{i}(s)\right)^{2} d s \leq|\omega|\|\overline{\mathbf{u}}\|_{W^{1, \infty}\left(\omega ; \mathbb{R}^{m}\right)}^{2} h^{2} .
\end{aligned}
$$


Therefore if

$$
h_{2}=\min \left\{h_{1}, \frac{\rho}{\|\overline{\mathbf{u}}\|_{W^{1, \infty}\left(\omega ; \mathbb{R}^{m}\right)}|\omega|^{1 / 2}}\right\}
$$

then $\Pi_{h} \overline{\mathbf{u}}$ belongs to $U_{a d, h} \cap B_{\rho}(\overline{\mathbf{u}})$ for all $h \leq h_{2}$. Now setting $\mathbf{u}_{h}=\Pi_{h} \overline{\mathbf{u}}$ and $\left(\mathbf{y}_{\mathbf{u}_{h}}^{h}, p_{\mathbf{u}_{h}}^{h}\right)=G_{h}\left(\mathbf{u}_{h}\right)$, we have that $\left(\mathbf{u}_{h}, \mathbf{y}_{\mathbf{u}_{h}}^{h}, p_{\mathbf{u}_{h}}^{h}\right)$ satisfies (4.3) and $\left(\mathbf{u}_{h}, \mathbf{y}_{\mathbf{u}_{h}}^{h}\right)$ is a feasible pair for $\left(\mathrm{P}_{h}\right)$ for any $h \leq h_{2}$.

Since the set of feasible points of $\left(\mathrm{P}_{h}\right)$ is nonempty and closed, and $F_{h}$ is continuous, convex on $U_{a d, h} \times \mathbf{X}_{h}$, and coercive with respect to $\mathbf{u} \in U_{a d, h}$, then $\left(\mathrm{P}_{h}\right)$ has at least one solution.

Now let us assume that $(\overline{\mathbf{u}}, \overline{\mathbf{y}})$ is a strict local solution of $(\mathrm{P})$ in $\left(U_{a d} \cap B_{\rho}(\overline{\mathbf{u}})\right) \times$ $B_{\rho}(\overline{\mathbf{y}})$. We consider the problems

$$
\left(\mathrm{Q}_{h}\right)\left\{\begin{array}{l}
\min J_{h}(\mathbf{u}), \\
\mathbf{u} \in U_{a d, h} \cap B_{\rho}(\overline{\mathbf{u}}),
\end{array}\right.
$$

where $J_{h}(\mathbf{u})=F\left(\mathbf{u}, \mathbf{y}_{\mathbf{u}}^{h}\right)$ with $\left(\mathbf{y}_{\mathbf{u}}^{h}, p_{\mathbf{u}}^{h}\right)=G_{h}(\mathbf{u}), G_{h}$ being defined in Remark 4.9. Above we have proved that $U_{a d, h} \cap B_{\rho}(\overline{\mathbf{u}})$ is nonempty for $h \leq h_{2}$. Observe that $U_{a d, h} \cap B_{\rho}(\overline{\mathbf{u}})$ is convex, bounded, and closed in $L^{2}\left(\omega ; \mathbb{R}^{m}\right)$, the mapping $\mathbf{u} \mapsto \int_{\omega}|\mathbf{u}|^{2}$ is lower semicontinuous for the weak topology of $L^{2}\left(\omega ; \mathbb{R}^{m}\right)$, and from Remark 4.9 it follows that the mapping $\mathbf{u} \mapsto \int_{\Omega}\left|\mathbf{y}_{\mathbf{u}}^{h}-\mathbf{y}_{d}\right|^{2}$ is continuous for the weak topology of $L^{2}\left(\omega ; \mathbb{R}^{m}\right)$. Therefore $\left(\mathrm{Q}_{h}\right)$ has at least one solution $\overline{\mathbf{u}}_{h}$. From any subsequence of $\left\{\overline{\mathbf{u}}_{h}\right\}_{h}$, we can extract another subsequence, still indexed by $h$ to simplify the notation, converging weakly in $L^{2}\left(\omega ; \mathbb{R}^{m}\right)$ to some $\tilde{\mathbf{u}} \in B_{\rho}(\overline{\mathbf{u}})$. Let us check that $\tilde{\mathbf{u}}=\overline{\mathbf{u}}$. Let us take again $\mathbf{u}_{h}=\Pi_{h} \overline{\mathbf{u}} \in U_{a d, h} \cap B_{\rho}(\overline{\mathbf{u}})$ for all $h<h_{2}$. By passing to the limit when $h$ tends to zero, with the convergence result stated in Lemma 4.10, we can write

$$
J(\tilde{\mathbf{u}}) \leq \liminf _{h \rightarrow 0} J_{h}\left(\overline{\mathbf{u}}_{h}\right) \leq \limsup _{h \rightarrow 0} J_{h}\left(\overline{\mathbf{u}}_{h}\right) \leq \limsup _{h \rightarrow 0} J_{h}\left(\Pi_{h} \overline{\mathbf{u}}\right)=J(\overline{\mathbf{u}}) .
$$

Since $\tilde{\mathbf{u}} \in B_{\rho}(\overline{\mathbf{u}})$ and the inequality in (4.6) is strict for $\mathbf{u} \neq \overline{\mathbf{u}}$, the above inequality implies that $\tilde{\mathbf{u}}=\overline{\mathbf{u}}$. Thus we have

$$
\lim _{h \rightarrow 0} J_{h}\left(\overline{\mathbf{u}}_{h}\right)=J(\overline{\mathbf{u}}),
$$

and still with Lemma 4.10, we deduce that

$$
\lim _{h \rightarrow 0} \int_{\omega}\left|\overline{\mathbf{u}}_{h}\right|^{2}=\int_{\omega}|\overline{\mathbf{u}}|^{2} .
$$

Therefore the subsequence $\left\{\overline{\mathbf{u}}_{h}\right\}_{h}$ converges to $\overline{\mathbf{u}}$ in $L^{2}\left(\omega ; \mathbb{R}^{m}\right)$. Since $\overline{\mathbf{u}}$ is the only cluster point for the weak topology of $L^{2}\left(\omega ; \mathbb{R}^{m}\right)$ of the original sequence $\left\{\overline{\mathbf{u}}_{h}\right\}_{h}$, it is clear that the convergence properties stated in the theorem hold for the whole sequence $\left\{\overline{\mathbf{u}}_{h}\right\}_{h}$. The convergence of the corresponding states is a consequence of Lemma 4.10. Finally, the strong convergence $\overline{\mathbf{u}}_{h} \rightarrow \overline{\mathbf{u}}$ in $L^{2}\left(\omega ; \mathbb{R}^{m}\right)$ implies that $\overline{\mathbf{u}}_{h}$ belongs to the interior of the ball $B_{\rho}^{h}(\overline{\mathbf{u}})$, which implies that $\left(\overline{\mathbf{u}}_{h}, \overline{\mathbf{y}}_{h}\right)$ is a local minimum of $\left(\mathrm{P}_{h}\right)$.

4.3. Discrete adjoint equation. We define the discrete adjoint state $\left(\Phi_{\mathbf{u}}^{h}, \pi_{\mathbf{u}}^{h}\right)$ $\in \mathbf{X}_{h} \times M_{h}$ associated with a control $\mathbf{u} \in B_{\rho_{2}}(\overline{\mathbf{u}})$ as the solution to the problem

$$
\begin{aligned}
& a\left(\boldsymbol{\Phi}_{\mathbf{u}}^{h}, \mathbf{w}_{h}\right)+b\left(\mathbf{y}_{\mathbf{u}}^{h}, \mathbf{w}_{h}, \boldsymbol{\Phi}_{\mathbf{u}}^{h}\right)+b\left(\mathbf{w}_{h}, \mathbf{y}_{\mathbf{u}}^{h}, \boldsymbol{\Phi}_{\mathbf{u}}^{h}\right)-\left(\pi_{\mathbf{u}}^{h}, \operatorname{div} \mathbf{w}_{h}\right) \\
& =\left(\mathbf{y}_{\mathbf{u}}^{h}-\mathbf{y}_{d}, \mathbf{w}_{h}\right) \quad \forall \mathbf{w}_{h} \in \mathbf{X}_{h}, \\
& \left(\lambda_{h}, \operatorname{div} \boldsymbol{\Phi}_{\mathbf{u}}^{h}\right)=0 \quad \forall \lambda_{h} \in M_{h} .
\end{aligned}
$$


Lemma 4.12. Let $(\overline{\mathbf{u}}, \overline{\mathbf{y}})$ be as in Theorem 4.7. There exist $0<h_{3} \leq h_{2}$ and $0<\rho_{3} \leq \rho_{2}$ such that, for all $\mathbf{u} \in B_{\rho_{3}}(\overline{\mathbf{u}})$ and all $0<h \leq h_{3}$, the system (4.8) admits a unique solution $\left(\boldsymbol{\Phi}_{\mathbf{u}}^{h}, \pi_{\mathbf{u}}^{h}\right) \in \mathbf{X}_{h} \times M_{h}$.

Proof. (i) For $\mathbf{y} \in \mathbf{H}_{0}^{1}(\Omega)$, consider the mapping $\mathcal{G}_{\mathbf{y}}$ from $\mathbf{H}_{0}^{1}(\Omega) \times L_{0}^{2}(\Omega)$ into itself defined by

$$
\mathcal{G}_{\mathbf{y}}(\boldsymbol{\Phi}, \pi)=(\boldsymbol{\Phi}, \pi)+T\left[B^{\prime}(\mathbf{y})^{*} \boldsymbol{\Phi}\right] .
$$

As in Lemma 4.2, we can easily show that $\mathcal{G}_{\mathbf{y}}$ is an automorphism in $\mathbf{H}_{0}^{1}(\Omega) \times L_{0}^{2}(\Omega)$ if and only if $\mathbf{y}$ is a nonsingular solution of (2.2). Thus $\mathcal{G}_{\overline{\mathbf{y}}}$ is an automorphism in $\mathbf{H}_{0}^{1}(\Omega) \times L_{0}^{2}(\Omega)$. We also introduce the mapping $\mathcal{G}_{\mathbf{y}, h}$ from $\mathbf{H}_{0}^{1}(\Omega) \times L_{0}^{2}(\Omega)$ into itself defined by

$$
\mathcal{G}_{\mathbf{y}, h}(\mathbf{\Phi}, \pi)=(\mathbf{\Phi}, \pi)+T_{h}\left[B^{\prime}(\mathbf{y})^{*} \mathbf{\Phi}\right] .
$$

Arguing as in the proof of Theorem 4.7, we can assume that $h_{0}$ is chosen so that, for all $0<h<h_{0}$ and all $\mathbf{y} \in B_{\rho_{0}}(\overline{\mathbf{y}}), \mathcal{G}_{\mathbf{y}, h}$ is an automorphism in $\mathbf{H}_{0}^{1}(\Omega) \times L_{0}^{2}(\Omega)$. In particular, according to estimate (4.5), there exist $0<h_{3} \leq h_{2}$ and $0<\rho_{3} \leq \rho_{2}$ such that, for all $0<h \leq h_{3}$ and all $\mathbf{u} \in B_{\rho_{3}}(\overline{\mathbf{u}}), \mathcal{G}_{\mathbf{y}_{\mathbf{u}}^{h}, h}$ is an automorphism in $\mathbf{H}_{0}^{1}(\Omega) \times L_{0}^{2}(\Omega)$ and

$$
\left\|\mathcal{G}_{\mathbf{y}_{\mathbf{u}}^{h}, h}^{-1}\right\|_{\mathcal{L}\left(\mathbf{H}_{0}^{1}(\Omega) \times L_{0}^{2}(\Omega)\right)} \leq 2\left\|\mathcal{G}_{\overline{\mathbf{y}}}^{-1}\right\|_{\mathcal{L}\left(\mathbf{H}_{0}^{1}(\Omega) \times L_{0}^{2}(\Omega)\right)} .
$$

Without loss of generality we can also assume that $\mathcal{G}_{\mathbf{y}(\mathbf{u})}$ is an automorphism in $\mathbf{H}_{0}^{1}(\Omega) \times L_{0}^{2}(\Omega)$ for all $\mathbf{u} \in B_{\rho_{3}}(\overline{\mathbf{u}})$.

(ii) Now we are going to show that $\left(\boldsymbol{\Phi}_{\mathbf{u}}^{h}, \pi_{\mathbf{u}}^{h}\right) \in \mathbf{X}_{h} \times M_{h}$ is a solution of (4.8) if and only if

$$
\mathcal{G}_{\mathbf{y}_{\mathbf{u}}^{h}, h}\left(\boldsymbol{\Phi}_{\mathbf{u}}^{h}, \pi_{\mathbf{u}}^{h}\right)=\left(\mathbf{z}_{h}, q_{h}\right),
$$

where $\left(\mathbf{z}_{h}, q_{h}\right) \in \mathbf{X}_{h} \times M_{h}$ is the solution of the discrete Stokes problem,

$$
\begin{aligned}
& a\left(\mathbf{z}_{h}, \mathbf{w}_{h}\right)-\left(q_{h}, \operatorname{div} \mathbf{w}_{h}\right)=\left(\mathbf{y}_{h}(\mathbf{u})-\mathbf{y}_{d}, \mathbf{w}_{h}\right) \forall \mathbf{w}_{h} \in \mathbf{X}_{h}, \\
& \left(\lambda_{h}, \operatorname{div} \mathbf{z}_{h}\right)=0 \forall \lambda_{h} \in M_{h} .
\end{aligned}
$$

To prove this result, we notice that (4.9) is satisfied if and only if

$$
\left(\boldsymbol{\Phi}_{\mathbf{u}}^{h}-\mathbf{z}_{h}, \pi_{\mathbf{u}}^{h}-q_{h}\right)=-T_{h}\left[B^{\prime}\left(\mathbf{y}_{\mathbf{u}}^{h}\right)^{*} \boldsymbol{\Phi}_{\mathbf{u}}^{h}\right],
$$

which is equivalent to

$$
\begin{aligned}
& a\left(\mathbf{\Phi}_{\mathbf{u}}^{h}-\mathbf{z}_{h}, \mathbf{w}_{h}\right)-\left(\pi_{\mathbf{u}}^{h}-q_{h}, \operatorname{div} \mathbf{w}_{h}\right)=\left(B^{\prime}\left(\mathbf{y}_{\mathbf{u}}^{h}\right)^{*} \boldsymbol{\Phi}_{\mathbf{u}}^{h}, \mathbf{w}_{h}\right) \forall \mathbf{w}_{h} \in \mathbf{X}_{h}, \\
& \left(\lambda_{h}, \operatorname{div} \mathbf{\Phi}_{\mathbf{u}}^{h}-\operatorname{div} \mathbf{z}_{h}\right)=0 \forall \lambda_{h} \in M_{h} .
\end{aligned}
$$

Now using equation (4.10), we see that (4.11) is equivalent to (4.8). This completes the proof.

We are going to prove error estimates for the discrete adjoint state. Set

$$
\mathbf{V}_{h}=\left\{\boldsymbol{\Phi}_{h} \in X_{h} \mid\left(\lambda_{h}, \operatorname{div} \boldsymbol{\Phi}_{h}\right)=0 \forall \lambda_{h} \in M_{h}\right\} .
$$

Lemma 4.13. Let $(\overline{\mathbf{u}}, \overline{\mathbf{y}})$ be as in Theorem 4.7. There exists a constant $C>0$ such that, for all $\mathbf{u}, \hat{\mathbf{u}} \in B_{\rho_{3}}(\overline{\mathbf{u}})$ and all $0<h<h_{3}$, the solution $\left(\Phi_{\mathbf{u}}, \pi_{\mathbf{u}}\right)$ to (3.8) 
and the solutions $\left(\boldsymbol{\Phi}_{\mathbf{u}}^{h}, \pi_{\mathbf{u}}^{h}\right)$ and $\left(\boldsymbol{\Phi}_{\hat{\mathbf{u}}}^{h}, \pi_{\hat{\mathbf{u}}}^{h}\right)$ to (4.8) obey the following estimates:

$$
\begin{aligned}
& \left\|\boldsymbol{\Phi}_{\mathbf{u}}-\boldsymbol{\Phi}_{\mathbf{u}}^{h}\right\|_{\mathbf{L}^{2}(\Omega)} \leq C h^{2}, \\
& \left\|\boldsymbol{\Phi}_{\mathbf{u}}-\boldsymbol{\Phi}_{\mathbf{u}}^{h}\right\|_{\mathbf{H}_{0}^{1}(\Omega)}+\left\|\pi_{\mathbf{u}}-\pi_{\mathbf{u}}^{h}\right\|_{L_{0}^{2}(\Omega)} \leq C h, \\
& \left\|\boldsymbol{\Phi}_{\mathbf{u}}-\boldsymbol{\Phi}_{\hat{\mathbf{u}}}^{h}\right\|_{\mathbf{H}_{0}^{1}(\Omega)}+\left\|\pi_{\mathbf{u}}-\pi_{\hat{\mathbf{u}}}^{h}\right\|_{L_{0}^{2}(\Omega)} \leq C\left(h+\|\mathbf{u}-\hat{\mathbf{u}}\|_{L^{2}\left(\omega ; \mathbb{R}^{m}\right)}\right) .
\end{aligned}
$$

Moreover, if $\mathbf{u}_{h} \in B_{\rho_{3}}(\overline{\mathbf{u}})$ and $\mathbf{u}_{h} \rightarrow \mathbf{u}$ weakly in $L^{2}\left(\omega ; \mathbb{R}^{m}\right)$, then $\boldsymbol{\Phi}_{\mathbf{u}}^{h} \rightarrow \boldsymbol{\Phi}_{\mathbf{u}}$ strongly in $C\left(\bar{\Omega} ; \mathbb{R}^{d}\right)$.

Proof. (i) We first show (4.13). From the proof of Lemma 4.12 it follows that $\mathcal{G}_{\mathbf{y}_{\mathbf{u}}}$ is an automorphism in $\mathbf{H}_{0}^{1}(\Omega) \times L_{0}^{2}(\Omega)$, and that, for all $\mathbf{u} \in B_{\rho_{3}}(\overline{\mathbf{u}})$ and all $0<h<h_{3}, \mathcal{G}_{\mathbf{y}_{\mathbf{u}}^{h}}$ is an automorphism in $\mathbf{H}_{0}^{1}(\Omega) \times L_{0}^{2}(\Omega)$ and

$$
\left\|\mathcal{G}_{\mathbf{y}_{\mathbf{u}}^{h}, h}^{-1}\right\|_{\mathcal{L}\left(\mathbf{H}_{0}^{1}(\Omega) \times L_{0}^{2}(\Omega)\right)} \leq 2\left\|\mathcal{G}_{\overline{\mathbf{y}}}^{-1}\right\|_{\mathcal{L}\left(\mathbf{H}_{0}^{1}(\Omega) \times L_{0}^{2}(\Omega)\right)} .
$$

Let us recall that $\left(\mathbf{\Phi}_{\mathbf{u}}, \pi_{\mathbf{u}}\right)$ is the solution of the equation

$$
\mathcal{G}_{\mathbf{y}_{\mathbf{u}}}\left(\Phi_{\mathbf{u}}, \pi_{\mathbf{u}}\right)=T\left(\mathbf{y}_{\mathbf{u}}-\mathbf{y}_{d}\right)
$$

and that $\left(\boldsymbol{\Phi}_{\mathbf{u}}^{h}, \pi_{\mathbf{u}}^{h}\right)$ is the solution of

$$
\mathcal{G}_{\mathbf{y}_{\mathbf{u}}^{h}, h}\left(\boldsymbol{\Phi}_{\mathbf{u}}^{h}, \pi_{\mathbf{u}}^{h}\right)=T_{h}\left(\mathbf{y}_{\mathbf{u}}^{h}-\mathbf{y}_{d}\right)
$$

Thus we have

$$
\begin{aligned}
& \mathcal{G}_{\mathbf{y}_{\mathbf{u}}^{h}, h}\left(\boldsymbol{\Phi}_{\mathbf{u}}-\boldsymbol{\Phi}_{\mathbf{u}}^{h}, \pi_{\mathbf{u}}-\pi_{\mathbf{u}}^{h}\right) \\
& =\left(\boldsymbol{\Phi}_{\mathbf{u}}, \pi_{\mathbf{u}}\right)+T_{h}\left(B^{\prime}\left(\mathbf{y}_{\mathbf{u}}^{h}\right)^{*} \boldsymbol{\Phi}_{\mathbf{u}}\right)-\mathcal{G}_{\mathbf{y}_{\mathbf{u}}^{h}, h}\left(\boldsymbol{\Phi}_{\mathbf{u}}^{h}, \pi_{\mathbf{u}}^{h}\right) \\
& =T_{h}\left[B^{\prime}\left(\mathbf{y}_{\mathbf{u}}^{h}\right)^{*} \boldsymbol{\Phi}_{\mathbf{u}}\right]-T\left[B^{\prime}\left(\mathbf{y}_{\mathbf{u}}\right)^{*} \boldsymbol{\Phi}_{\mathbf{u}}\right]+T\left(\mathbf{y}_{\mathbf{u}}-\mathbf{y}_{d}\right)-T_{h}\left(\mathbf{y}_{\mathbf{u}}^{h}-\mathbf{y}_{d}\right) \\
& =\left(T-T_{h}\right)\left[\mathbf{y}_{\mathbf{u}}^{h}-B^{\prime}\left(\mathbf{y}_{\mathbf{u}}^{h}\right)^{*} \boldsymbol{\Phi}_{\mathbf{u}}-\mathbf{y}_{d}\right] \\
& \quad+T\left[B^{\prime}\left(\mathbf{y}_{\mathbf{u}}^{h}\right)^{*} \boldsymbol{\Phi}_{\mathbf{u}}-B^{\prime}\left(\mathbf{y}_{\mathbf{u}}\right)^{*} \boldsymbol{\Phi}_{\mathbf{u}}\right]+T\left[\mathbf{y}_{\mathbf{u}}-\mathbf{y}_{\mathbf{u}}^{h}\right],
\end{aligned}
$$

which yields

$$
\begin{aligned}
& \left(\boldsymbol{\Phi}_{\mathbf{u}}-\boldsymbol{\Phi}_{\mathbf{u}}^{h}, \pi_{\mathbf{u}}-\pi_{\mathbf{u}}^{h}\right) \\
& \begin{aligned}
=\left(\mathcal{G}_{\mathbf{y}_{\mathbf{u}}^{h}, h}\right)^{-1} & \left(\left(T-T_{h}\right)\left[\mathbf{y}_{\mathbf{u}}^{h}-B^{\prime}\left(\mathbf{y}_{\mathbf{u}}^{h}\right)^{*} \boldsymbol{\Phi}_{\mathbf{u}}-\mathbf{y}_{d}\right]\right. \\
& \left.+T\left[B^{\prime}\left(\mathbf{y}_{\mathbf{u}}^{h}\right)^{*} \mathbf{\Phi}_{\mathbf{u}}-B^{\prime}\left(\mathbf{y}_{\mathbf{u}}\right)^{*} \boldsymbol{\Phi}_{\mathbf{u}}\right]+T\left[\mathbf{y}_{\mathbf{u}}-\mathbf{y}_{\mathbf{u}}^{h}\right]\right) .
\end{aligned}
\end{aligned}
$$

With estimate (4.5) and assumption (S2), we obtain

$$
\left\|\Phi_{\mathbf{u}}-\boldsymbol{\Phi}_{\mathbf{u}}^{h}\right\|_{\mathbf{H}_{0}^{1}(\Omega)}+\left\|\pi_{\mathbf{u}}-\pi_{\mathbf{u}}^{h}\right\|_{L_{0}^{2}(\Omega)} \leq C h .
$$

(ii) To prove (4.12) we proceed as in [12, Chapter 2, Theorems 1.2 and 1.9]. The solution $\left(\boldsymbol{\Phi}_{\mathbf{u}}, \pi_{\mathbf{u}}\right)$ to (3.8) and the solution $\left(\boldsymbol{\Phi}_{\mathbf{u}}^{h}, \pi_{\mathbf{u}}^{h}\right)$ to (4.8) satisfy

$$
\begin{aligned}
& a\left(\boldsymbol{\Phi}_{\mathbf{u}}-\boldsymbol{\Phi}_{\mathbf{u}}^{h}, \mathbf{w}_{h}\right)+b\left(\mathbf{y}_{\mathbf{u}}, \mathbf{w}_{h}, \boldsymbol{\Phi}_{\mathbf{u}}-\boldsymbol{\Phi}_{\mathbf{u}}^{h}\right)+b\left(\mathbf{w}_{h}, \mathbf{y}_{\mathbf{u}}, \boldsymbol{\Phi}_{\mathbf{u}}-\boldsymbol{\Phi}_{\mathbf{u}}^{h}\right) \\
& -\left(\pi_{\mathbf{u}}-\pi_{\mathbf{u}}^{h}, \operatorname{div} \mathbf{w}_{h}\right)=\left(\mathbf{y}_{\mathbf{u}}-\mathbf{y}_{\mathbf{u}}^{h}, \mathbf{w}_{h}\right)+b\left(\mathbf{y}_{\mathbf{u}}-\mathbf{y}_{\mathbf{u}}^{h}, \mathbf{w}_{h}, \boldsymbol{\Phi}_{\mathbf{u}}^{h}\right) \\
& +b\left(\mathbf{w}_{h}, \mathbf{y}_{\mathbf{u}}-\mathbf{y}_{\mathbf{u}}^{h}, \boldsymbol{\Phi}_{\mathbf{u}}^{h}\right) \forall \mathbf{w}_{h} \in \mathbf{X}_{h}, \\
& \left(\lambda_{h}, \operatorname{div} \boldsymbol{\Phi}_{\mathbf{u}}-\operatorname{div} \boldsymbol{\Phi}_{\mathbf{u}}^{h}\right)=0 \forall \lambda_{h} \in M_{h} .
\end{aligned}
$$


For all $\mathbf{g} \in \mathbf{L}^{2}(\Omega)$, let us consider the solution $\left(\mathbf{z}_{\mathbf{g}}, q_{\mathbf{g}}\right) \in \mathbf{H}_{0}^{1}(\Omega) \times L_{0}^{2}(\Omega)$ to

$$
\begin{aligned}
& a\left(\mathbf{z}_{\mathbf{g}}, \mathbf{w}\right)+b\left(\mathbf{y}_{\mathbf{u}}, \mathbf{z}_{\mathbf{g}}, \mathbf{w}\right)+b\left(\mathbf{z}_{\mathbf{g}}, \mathbf{y}_{\mathbf{u}}, \mathbf{w}\right)-\left(q_{\mathbf{g}}, \operatorname{div} \mathbf{w}\right)=(\mathbf{g}, \mathbf{w}) \forall \mathbf{w} \in \mathbf{H}_{0}^{1}(\Omega), \\
& \left(\lambda, \operatorname{div} \mathbf{z}_{\mathbf{g}}\right)=0 \forall \lambda \in L_{0}^{2}(\Omega),
\end{aligned}
$$

and the solution $\left(\mathbf{z}_{\mathbf{g}}^{h}, q_{\mathbf{g}}^{h}\right) \in \mathbf{H}_{0}^{1}(\Omega) \times L_{0}^{2}(\Omega)$ to

$$
a\left(\mathbf{z}_{\mathbf{g}}^{h}, \mathbf{w}_{h}\right)+b\left(\mathbf{y}_{\mathbf{u}}, \mathbf{z}_{\mathbf{g}}^{h}, \mathbf{w}_{h}\right)+b\left(\mathbf{z}_{\mathbf{g}}^{h}, \mathbf{y}_{\mathbf{u}}, \mathbf{w}_{h}\right)-\left(q_{\mathbf{g}}^{h}, \operatorname{div} \mathbf{w}_{h}\right)=\left(\mathbf{g}, \mathbf{w}_{h}\right) \forall \mathbf{w} \in \mathbf{X}_{h},
$$

$\left(\lambda, \operatorname{div} \mathbf{z}_{\mathbf{g}}^{h}\right)=0 \forall \lambda \in M_{h}$.

Choosing $\mathbf{w}_{h}=\mathbf{z}_{\mathbf{g}}^{h}$ in (4.15) and $\mathbf{w}=\boldsymbol{\Phi}_{\mathbf{u}}-\boldsymbol{\Phi}_{\mathbf{u}}^{h}$ in (4.16) and combining the two identities, we obtain

$$
\begin{aligned}
& \left(\mathbf{g}, \boldsymbol{\Phi}_{\mathbf{u}}-\boldsymbol{\Phi}_{\mathbf{u}}^{h}\right)=a\left(\boldsymbol{\Phi}_{\mathbf{u}}-\boldsymbol{\Phi}_{\mathbf{u}}^{h}, \mathbf{z}_{\mathbf{g}}-\mathbf{z}_{\mathbf{g}}^{h}\right)+b\left(\mathbf{y}_{\mathbf{u}}, \mathbf{z}_{\mathbf{g}}-\mathbf{z}_{\mathbf{g}}^{h}, \boldsymbol{\Phi}_{\mathbf{u}}-\boldsymbol{\Phi}_{\mathbf{u}}^{h}\right) \\
& +b\left(\mathbf{z}_{\mathbf{g}}-\mathbf{z}_{\mathbf{g}}^{h}, \mathbf{y}_{\mathbf{u}}, \boldsymbol{\Phi}_{\mathbf{u}}-\boldsymbol{\Phi}_{\mathbf{u}}^{h}\right)+\left(\pi_{\mathbf{u}}-\pi_{\mathbf{u}}^{h}, \operatorname{div} \mathbf{z}_{\mathbf{g}}^{h}\right)-\left(q_{\mathbf{g}}, \operatorname{div} \boldsymbol{\Phi}_{\mathbf{u}}-\operatorname{div} \boldsymbol{\Phi}_{\mathbf{u}}^{h}\right) \\
& +\left(\mathbf{y}_{\mathbf{u}}-\mathbf{y}_{\mathbf{u}}^{h}, \mathbf{z}_{\mathbf{g}}^{h}\right)+b\left(\mathbf{y}_{\mathbf{u}}-\mathbf{y}_{\mathbf{u}}^{h}, \mathbf{z}_{\mathbf{g}}^{h}, \boldsymbol{\Phi}_{\mathbf{u}}^{h}\right)+b\left(\mathbf{z}_{\mathbf{g}}^{h}, \mathbf{y}_{\mathbf{u}}-\mathbf{y}_{\mathbf{u}}^{h}, \boldsymbol{\Phi}_{\mathbf{u}}^{h}\right) \\
& =a\left(\boldsymbol{\Phi}_{\mathbf{u}}-\boldsymbol{\Phi}_{\mathbf{u}}^{h}, \mathbf{z}_{\mathbf{g}}-\mathbf{z}_{\mathbf{g}}^{h}\right)-b\left(\mathbf{y}_{\mathbf{u}}, \boldsymbol{\Phi}_{\mathbf{u}}-\boldsymbol{\Phi}_{\mathbf{u}}^{h}, \mathbf{z}_{\mathbf{g}}-\mathbf{z}_{\mathbf{g}}^{h}\right)-b\left(\mathbf{z}_{\mathbf{g}}-\mathbf{z}_{\mathbf{g}}^{h}, \boldsymbol{\Phi}_{\mathbf{u}}-\boldsymbol{\Phi}_{\mathbf{u}}^{h}, \mathbf{y}_{\mathbf{u}}\right) \\
& +\left(\mathbf{y}_{\mathbf{u}}-\mathbf{y}_{\mathbf{u}}^{h}, \mathbf{z}_{\mathbf{g}}^{h}\right)-b\left(\mathbf{y}_{\mathbf{u}}-\mathbf{y}_{\mathbf{u}}^{h}, \boldsymbol{\Phi}_{\mathbf{u}}^{h}, \mathbf{z}_{\mathbf{g}}^{h}\right)-b\left(\mathbf{z}_{\mathbf{g}}^{h}, \boldsymbol{\Phi}_{\mathbf{u}}^{h}, \mathbf{y}_{\mathbf{u}}-\mathbf{y}_{\mathbf{u}}^{h}\right) \\
& +\left(\pi_{\mathbf{u}}-\pi_{\mathbf{u}}^{h}, \operatorname{div} \mathbf{z}_{\mathbf{g}}^{h}-\operatorname{div} \mathbf{z}_{\mathbf{g}}\right)-\left(q_{\mathbf{g}}-q_{\mathbf{g}}^{h}, \operatorname{div} \boldsymbol{\Phi}_{\mathbf{u}}-\operatorname{div} \boldsymbol{\Phi}_{\mathbf{u}}^{h}\right) .
\end{aligned}
$$

Thus we have

$$
\begin{aligned}
& \left\|\boldsymbol{\Phi}_{\mathbf{u}}-\boldsymbol{\Phi}_{\mathbf{u}}^{h}\right\|_{\mathbf{L}^{2}(\Omega)}=\sup _{\|\mathbf{g}\|_{\mathbf{L}^{2}(\Omega)}=1}\left(\mathbf{g}, \boldsymbol{\Phi}_{\mathbf{u}}-\boldsymbol{\Phi}_{\mathbf{u}}^{h}\right) \\
& \leq C \sup _{\|\mathbf{g}\|_{\mathbf{L}^{2}(\Omega)}=1}\left\{\left\|\boldsymbol{\Phi}_{\mathbf{u}}-\boldsymbol{\Phi}_{\mathbf{u}}^{h}\right\|_{\mathbf{H}_{0}^{1}(\Omega)}\left\|\mathbf{z}_{\mathbf{g}}-\mathbf{z}_{\mathbf{g}}^{h}\right\|_{\mathbf{H}_{0}^{1}(\Omega)}\right. \\
& \quad+\left\|\boldsymbol{\Phi}_{\mathbf{u}}-\boldsymbol{\Phi}_{\mathbf{u}}^{h}\right\|_{\mathbf{H}_{0}^{1}(\Omega)}\left\|\mathbf{z}_{\mathbf{g}}-\mathbf{z}_{\mathbf{g}}^{h}\right\|_{\mathbf{L}^{2}(\Omega)}\left\|\mathbf{y}_{\mathbf{u}}\right\|_{\mathbf{L}^{\infty}(\Omega)} \\
& \quad+\left\|\mathbf{y}_{\mathbf{u}}-\mathbf{y}_{\mathbf{u}}^{h}\right\|_{\mathbf{L}^{2}(\Omega)}\left\|\mathbf{z}_{\mathbf{g}}^{h}\right\|_{\mathbf{L}^{2}(\Omega)}+\left\|\mathbf{y}_{\mathbf{u}}-\mathbf{y}_{\mathbf{u}}^{h}\right\|_{\mathbf{L}^{2}(\Omega)}\left\|\mathbf{z}_{\mathbf{g}}^{h}\right\|_{\mathbf{L}^{\infty}(\Omega)}\left\|\boldsymbol{\Phi}_{\mathbf{u}}^{h}\right\|_{\mathbf{H}_{0}^{1}(\Omega)} \\
& \left.\quad+\left\|\pi_{\mathbf{u}}-\pi_{\mathbf{u}}^{h}\right\|_{L_{0}^{2}(\Omega)}\left\|\mathbf{z}_{\mathbf{g}}-\mathbf{z}_{\mathbf{g}}^{h}\right\|_{\mathbf{H}_{0}^{1}(\Omega)}+\left\|q_{\mathbf{g}}-q_{\mathbf{g}}^{h}\right\|_{L_{0}^{2}(\Omega)}\left\|\boldsymbol{\Phi}_{\mathbf{u}}-\mathbf{\Phi}_{\mathbf{u}}^{h}\right\|_{\mathbf{H}_{0}^{1}(\Omega)}\right\} .
\end{aligned}
$$

To complete estimate (4.12), we are going to use (4.13) and a similar error estimate for $\left(\mathbf{z}_{\mathbf{g}}, q_{\mathbf{g}}\right)$ :

$$
\left\|\mathbf{z}_{\mathbf{g}}-\mathbf{z}_{\mathbf{g}}^{h}\right\|_{\mathbf{H}_{0}^{1}(\Omega)}+\left\|q_{\mathbf{g}}-q_{\mathbf{g}}^{h}\right\|_{L_{0}^{2}(\Omega)} \leq C h\left(\left\|\mathbf{z}_{\mathbf{g}}\right\|_{\mathbf{H}^{2}(\Omega)}+\left\|q_{\mathbf{g}}\right\|_{H^{1}(\Omega)}\right) .
$$

With (4.17), (4.13), (4.18), and (4.4), we obtain

$$
\left\|\Phi_{\mathbf{u}}-\boldsymbol{\Phi}_{\mathbf{u}}^{h}\right\|_{\mathbf{L}^{2}(\Omega)} \leq C h^{2} .
$$

The proof of (4.12) is complete. Estimate (4.14) and the last statement in the lemma can now be proved in the same way as we did it for the state.

Let $(\overline{\mathbf{u}}, \overline{\mathbf{y}})$ be a nonsingular strict local minimum of $(\mathrm{P})$ and $\left\{\left(\overline{\mathbf{u}}_{h}, \overline{\mathbf{y}}_{h}\right)\right\}_{h<h_{3}}$ be a sequence of local minima of problems $\left(\mathrm{P}_{h}\right)$ converging to $(\overline{\mathbf{u}}, \overline{\mathbf{y}})$ in $\mathbf{L}^{2}\left(\omega ; \mathbb{R}^{m}\right) \times \mathbf{H}_{0}^{1}(\Omega)$, with $\overline{\mathbf{u}}_{h} \in B_{\rho_{3}}(\overline{\mathbf{u}})$, where $h_{3}$ and $\rho_{3}$ are given by Lemma 4.12 . Then every element $\overline{\mathbf{u}}_{h}$ from a sequence $\left\{\overline{\mathbf{u}}_{h}\right\}_{h \leq h_{3}}$ is a local solution of the problem

$$
\left(\hat{\mathrm{P}}_{h}\right)\left\{\begin{array}{l}
\min J_{h}(\mathbf{u})=F\left(\mathbf{u}, \mathbf{y}_{\mathbf{u}}^{h}\right), \\
\mathbf{u} \in U_{a d, h}
\end{array}\right.
$$


where $\left(\mathbf{y}_{\mathbf{u}}^{h}, p_{\mathbf{u}}^{h}\right)=G_{h}(\mathbf{u}), G_{h}$ being defined in Remark 4.9 .

LEMmA 4.14. Let $\overline{\mathbf{u}}_{h}$ be a solution to problem $\left(\hat{\mathrm{P}}_{h}\right)$, and let $\left(\overline{\mathbf{y}}_{h}, \bar{p}_{h}\right) \in \mathbf{X}_{h} \times M_{h}$ be the corresponding state and pressure. Then $\overline{\mathbf{u}}_{h}$ satisfies

$$
\int_{\omega}\left(\mathcal{C}^{*} \overline{\mathbf{\Phi}}_{h}+N \overline{\mathbf{u}}_{h}\right) \cdot\left(\mathbf{u}_{h}-\overline{\mathbf{u}}_{h}\right) d x \geq 0 \forall \mathbf{u}_{h} \in U_{a d, h},
$$

where $\left(\overline{\mathbf{\Phi}}_{h}, \bar{\pi}_{h}\right)=\left(\boldsymbol{\Phi}_{\overline{\mathbf{u}}_{h}}^{h}, \pi_{\overline{\mathbf{u}}_{h}}^{h}\right) \in \mathbf{X}_{h} \times M_{h}$ is the discrete adjoint state associated with $\overline{\mathbf{u}}_{h}$, that is, the solution to the system (4.8) where $\mathbf{u}$ is replaced by $\overline{\mathbf{u}}_{h}$.

Proof. The lemma is a consequence of the following identity:

$$
J_{h}^{\prime}\left(\overline{\mathbf{u}}_{h}\right)\left(\mathbf{u}_{h}-\overline{\mathbf{u}}_{h}\right)=\int_{\omega}\left(\mathcal{C}^{*} \overline{\mathbf{\Phi}}_{h}+N \overline{\mathbf{u}}_{h}\right) \cdot\left(\mathbf{u}_{h}-\overline{\mathbf{u}}_{h}\right) d x
$$

Now we can establish uniform convergence for the controls.

Lemma 4.15. Let $\overline{\mathbf{u}}_{h}$ be as in Lemma 4.14; then $\lim _{h \rightarrow 0}\left\|\overline{\mathbf{u}}_{h}-\overline{\mathbf{u}}\right\|_{L^{\infty}\left(\omega ; \mathbb{R}^{m}\right)}=0$.

Proof. Let us start with the case where $U_{a d, h}=U_{a d}^{h}$. Since the components of the elements of $U_{h}$ are constant on every triangle, for all $T \in \mathcal{T}_{h}$ and $1 \leq i \leq m$, we have

$$
\left.\bar{u}_{i, h}\right|_{T}=\operatorname{Proj}_{\left[\alpha_{i}, \beta_{i}\right]}\left(-\frac{1}{N|T|} \int_{T}\left(\mathcal{C}^{*} \overline{\mathbf{\Phi}}_{h}\right)_{i}(x) d x\right) .
$$

For all $x \in T$, using (3.9), the integral mean value theorem, and the Lipschitz continuity of $\overline{\mathbf{\Phi}}$, we can write

$$
\begin{aligned}
& \left|\bar{u}_{i, h}(x)-\bar{u}_{i}(x)\right| \leq\left|\frac{1}{N|T|} \int_{T}\left(\mathcal{C}^{*} \overline{\mathbf{\Phi}}_{h}\right)_{i}(s) d s-\frac{1}{N}\left(\mathcal{C}^{*} \overline{\mathbf{\Phi}}\right)_{i}(x)\right| \\
& =\frac{1}{N}\left|\left(\mathcal{C}^{*} \overline{\mathbf{\Phi}}_{h}\right)_{i}\left(x_{T}\right)-\left(\mathcal{C}^{*} \overline{\mathbf{\Phi}}\right)_{i}(x)\right| \\
& \leq \frac{1}{N}\left|\left(\mathcal{C}^{*} \overline{\mathbf{\Phi}}_{h}\right)_{i}\left(x_{T}\right)-\left(\mathcal{C}^{*} \overline{\mathbf{\Phi}}\right)_{i}\left(x_{T}\right)\right|+\frac{1}{N}\left|\left(\mathcal{C}^{*} \overline{\mathbf{\Phi}}\right)_{i}\left(x_{T}\right)-\left(\mathcal{C}^{*} \overline{\mathbf{\Phi}}\right)_{i}(x)\right| \\
& \leq C\left\|\overline{\mathbf{\Phi}}_{h}-\overline{\mathbf{\Phi}}\right\|_{\mathbf{L}^{\infty}(\Omega)}+C\left|x_{T}-x\right| \leq C\left(\left\|\overline{\mathbf{\Phi}}_{h}-\overline{\mathbf{\Phi}}\right\|_{\mathbf{L}^{\infty}(\Omega)}+h\right)
\end{aligned}
$$

for some $x_{T} \in T$. The uniform convergence of the adjoint states allows us to complete the proof in the case when $U_{a d, h}=U_{a d}^{h}$.

In the case when $U_{a d, h}=U_{a d}$ we have

$$
\bar{u}_{i, h}(x)=\operatorname{Proj}_{\left[\alpha_{i}, \beta_{i}\right]}\left(-\frac{1}{N|T|}\left(\mathcal{C}^{*} \overline{\mathbf{\Phi}}_{h}\right)_{i}(x)\right) .
$$

The convergence of $\overline{\mathbf{u}}_{h}$ follows from Lemma 4.13.

4.4. Error estimates. Let $(\overline{\mathbf{u}}, \overline{\mathbf{y}})$ be a nonsingular local solution of $(\mathrm{P})$ satisfying the sufficient second order optimality conditions (3.15) or, equivalently, (3.25). As a consequence of these conditions, we know that $(\overline{\mathbf{u}}, \overline{\mathbf{y}})$ is a strict local minimum of $(\mathrm{P})$. Let $\left\{\left(\overline{\mathbf{u}}_{h}, \overline{\mathbf{y}}_{h}\right)\right\}_{h}$ be a sequence of local solutions of problems $\left(\mathrm{P}_{h}\right)$ converging to $(\overline{\mathbf{u}}, \overline{\mathbf{y}})$; see Theorem 4.11 and Lemma 4.15. We assume that $h \leq h_{3}$ and $\overline{\mathbf{u}}_{h} \in B_{\rho_{3}}(\overline{\mathbf{u}})$, so that $\overline{\mathbf{u}}_{h}$ is a local minimum of $\left(\hat{\mathrm{P}}_{h}\right)$. The goal of this section is to estimate the order of convergence of this sequence.

Lemma 4.16. Let $\delta>0$ be the constant defined in Corollary 3.11. There exists $0<h_{4} \leq h_{3}$ such that

$$
\frac{\delta}{2}\left\|\overline{\mathbf{u}}-\overline{\mathbf{u}}_{h}\right\|_{L^{2}\left(\omega ; \mathbb{R}^{m}\right)}^{2} \leq\left(J^{\prime}\left(\overline{\mathbf{u}}_{h}\right)-J^{\prime}(\overline{\mathbf{u}})\right)\left(\overline{\mathbf{u}}_{h}-\overline{\mathbf{u}}\right) \forall 0<h<h_{4} .
$$


Proof. First, let us check that for $h>0$ small enough, $\overline{\mathbf{u}}_{h}-\overline{\mathbf{u}}$ belongs to $C_{\overline{\mathbf{u}}}^{\tau}$. The sign condition (3.21)-(3.22) is trivial since $\overline{\mathbf{u}}_{h} \in U_{a d}$. We have to check condition (3.20). Let us set

$$
\overline{\mathbf{d}}_{h}(x)=\left(\mathcal{C}^{*} \overline{\mathbf{\Phi}}_{h}\right)(x)+N \overline{\mathbf{u}}_{h}(x) .
$$

Take $h_{4}$ small enough to have

$$
\left\|\overline{\mathbf{d}}-\overline{\mathbf{d}}_{h}\right\|_{L^{\infty}(\omega)}<\frac{\tau}{4}, \quad \text { and } \quad\left\|\overline{\mathbf{d}}\left(x_{1}\right)-\overline{\mathbf{d}}\left(x_{2}\right)\right\|_{\mathbb{R}^{m}}<\frac{\tau}{4} \quad \text { if } \quad\left\|x_{1}-x_{2}\right\|_{\mathbb{R}^{d}}<h,
$$

for all $0<h \leq h_{4}$. First consider the case where $U_{a d, h}=U_{a d}$. In that case, if $\bar{d}_{i}(\xi)>\tau$ (respectively, $\bar{d}_{i}(\xi)<-\tau$ ), we have $\bar{d}_{i, h}(\xi)>3 \tau / 4$ (respectively, $\left.\bar{d}_{i, h}(\xi)<-3 \tau / 4\right)$, and $\bar{u}_{i}(\xi)=\alpha_{i}$ and $\bar{u}_{i, h}(\xi)=\alpha_{i}>-\infty\left(\right.$ respectively, $\bar{u}_{i}(\xi)=\beta_{i}$ and $\bar{u}_{i, h}(\xi)=\beta_{i}<$ $\infty)$. Thus $\mathbf{u}_{i, h}(\xi)=\mathbf{u}_{i}(\xi)$ if $\left|\bar{d}_{i}(\xi)\right|>\tau$, and condition (3.20) is satisfied.

Now consider the case where $U_{a d, h}=U_{a d}^{h}$. For all $T \in \mathcal{T}_{h}$ and all $1 \leq i \leq m$ let us set

$$
I_{i, T}=\int_{T} \bar{d}_{i, h}(x) d x
$$

Take $\xi \in \omega$ such that $\bar{d}_{i}(\xi)>\tau$. In this case $\bar{u}_{i}(\xi)=\alpha_{i}>-\infty$. Choose $x$ in the same triangle $T$ as $\xi$. Then

$$
\bar{d}_{i, h}(x)=\bar{d}_{i, h}(x)-\bar{d}_{i}(x)+\bar{d}_{i}(x)-\bar{d}_{i}(\xi)+\bar{d}_{i}(\xi)>-\frac{\tau}{4}-\frac{\tau}{4}+\tau=\frac{\tau}{2} .
$$

Therefore $I_{i, T}>0$ and $\left.\bar{u}_{i, h}\right|_{T}=\alpha_{i}$. In particular $\bar{u}_{i, h}(\xi)=\alpha_{i}$ and $\bar{u}_{i, h}(\xi)-\bar{u}_{i}(\xi)=0$. Similarly if $\bar{d}_{i}(\xi)<-\tau$, we have $\bar{u}_{i, h}(\xi)=\beta_{i}<\infty$ and $\bar{u}_{i, h}(\xi)-\bar{u}_{i}(\xi)=0$, and condition (3.20) is still satisfied in that case.

Thus second order sufficient conditions stated in Corollary 3.11 can be applied, and we have

$$
J^{\prime \prime}(\overline{\mathbf{u}})\left(\overline{\mathbf{u}}_{h}-\overline{\mathbf{u}}\right)^{2} \geq \delta\left\|\overline{\mathbf{u}}_{h}-\overline{\mathbf{u}}\right\|_{L^{2}\left(\omega ; \mathbb{R}^{m}\right)}^{2} .
$$

On the other hand, with the mean value theorem, we obtain

$$
\left(J^{\prime}\left(\overline{\mathbf{u}}_{h}\right)-J^{\prime}(\overline{\mathbf{u}})\right)\left(\overline{\mathbf{u}}_{h}-\overline{\mathbf{u}}\right)=J^{\prime \prime}\left(\overline{\mathbf{u}}+\theta_{h}\left(\overline{\mathbf{u}}-\overline{\mathbf{u}}_{h}\right)\right)\left(\overline{\mathbf{u}}_{h}-\overline{\mathbf{u}}\right)^{2}
$$

for some $0<\theta_{h}<1$. Due to the uniform convergence properties stated for the control and the adjoint state and the explicit form of the second derivative of $J$, it is clear that we can choose $h_{4}$ small enough to have

$$
J^{\prime \prime}\left(\overline{\mathbf{u}}+\theta_{h}\left(\overline{\mathbf{u}}-\overline{\mathbf{u}}_{h}\right)\right)\left(\overline{\mathbf{u}}_{h}-\overline{\mathbf{u}}\right)^{2} \geq \frac{\delta}{2}\left\|\overline{\mathbf{u}}_{h}-\overline{\mathbf{u}}\right\|_{L^{2}\left(\omega ; \mathbb{R}^{m}\right)}^{2}
$$

for all $0<h \leq h_{4}$. The proof is complete.

Lemma 4.17. Assume that $U_{a d, h}=U_{a d}^{h}$. There exists $0<h_{5} \leq h_{4}$ such that for every $0<h \leq h_{5}$ there exist $\mathbf{u}_{h}^{*} \in U_{h}$ and a constant $C>0$ independent of $h$ such that

(1) $\mathbf{u}_{h}^{*} \in U_{a d, h}$,

(2) $J^{\prime}(\overline{\mathbf{u}}) \overline{\mathbf{u}}=J^{\prime}(\overline{\mathbf{u}}) \mathbf{u}_{h}^{*}$

(3) $\left\|\overline{\mathbf{u}}-\mathbf{u}_{h}^{*}\right\|_{L^{\infty}\left(\omega ; \mathbb{R}^{m}\right)} \leq C h$. 
Proof. For every triangle $T \in \mathcal{T}_{h}$ and $1 \leq i \leq m$, define

$$
I_{i, T}=\int_{T} \bar{d}_{i}(x) d x
$$

and

$$
\left.u_{i, h}^{*}\right|_{T}= \begin{cases}\frac{1}{I_{i, T}} \int_{T} d_{i}(x) \bar{u}_{i}(x) d x & \text { if } I_{i, T} \neq 0, \\ \frac{1}{|T|} \int_{T} \bar{u}_{i}(x) d x & \text { if } I_{i, T}=0 .\end{cases}
$$

Due to the Lipschitz continuity of $\overline{\mathbf{u}}$, there exists $0<h_{5} \leq h_{4}$ such that, for $0<$ $h \leq h_{5}$, each component $\bar{u}_{i}$ cannot achieve both values $\alpha$ and $\beta$ in the same triangle. Hence, for each $T \in \mathcal{T}_{h}$, either $\bar{d}_{i}(x)$ is nonnegative for all $x \in T$ or $\bar{d}_{i}(x)$ is nonpositive for all $x \in T$. Therefore, $I_{i, T}=0$ if and only if $\bar{d}_{i}(x)=0$ for all $x \in T$. Moreover, if $I_{i, T} \neq 0$, then $\bar{d}_{i}(x) / I_{i, T} \geq 0$ for all $x \in T$. So applying the integral mean value theorem if $I_{i, T}=0$ or the generalized mean value theorem if $I_{i, T} \neq 0$, we have $\left.u_{i, h}^{*}\right|_{T}=\bar{u}_{i}\left(x_{T}\right)$ for some $x_{T} \in T$. As a first consequence, $\mathbf{u}_{h}^{*} \in U_{a d, h}$. Moreover, due to the Lipschitz continuity of $\overline{\mathbf{u}}$, we have that for $x \in \omega$, if we fix the triangle $T$ such that $x \in T$,

$$
\left|\bar{u}_{i}(x)-u_{i, h}^{*}(x)\right|=\left|\bar{u}_{i}(x)-\bar{u}_{i}\left(x_{T}^{i}\right)\right| \leq C\left\|x-x_{T}^{i}\right\|_{\mathbb{R}^{d}} \leq C h,
$$

and we have proved statement 3 .

Since $I_{i, T}=0$ if and only if $\bar{d}_{i}(x)=0$ for all $x \in T$, we can claim that

$$
\left.I_{i, T} u_{i, h}^{*}\right|_{T}=\int_{T} \bar{d}_{i}(x) \bar{u}_{i}(x) d x
$$

for all $T \in \mathcal{T}_{h}$ and all $1 \leq i \leq m$. A straightforward calculation yields statement 2 :

$$
\begin{aligned}
& J^{\prime}(\overline{\mathbf{u}}) \mathbf{u}_{h}^{*}=\int_{\omega} \overline{\mathbf{d}}(x) \cdot \overline{\mathbf{u}}_{h}^{*}(x) d x=\sum_{i=1}^{m} \sum_{T \in \mathcal{T}_{h}} \int_{T} \bar{d}_{i}(x) u_{i, h}^{*}(x) d x \\
& =\left.\sum_{i=1}^{m} \sum_{T \in \mathcal{T}_{h}} I_{i, T} u_{i, h}^{*}\right|_{T}=\sum_{i=1}^{m} \sum_{T \in \mathcal{T}_{h}} \int_{T} \bar{d}_{i}(x) \bar{u}_{i}(x) d x=J^{\prime}(\overline{\mathbf{u}}) \overline{\mathbf{u}} .
\end{aligned}
$$

THEOREM 4.18. There exists a constant $C>0$ such that, for all $0<h \leq h_{5}$, we have

$$
\left\|\overline{\mathbf{u}}-\overline{\mathbf{u}}_{h}\right\|_{L^{2}\left(\omega ; \mathbb{R}^{m}\right)} \leq C h^{2} \quad \text { if } \quad U_{a d, h}=U_{a d}
$$

while

$$
\left\|\overline{\mathbf{u}}-\overline{\mathbf{u}}_{h}\right\|_{L^{2}\left(\omega ; \mathbb{R}^{m}\right)} \leq C h \quad \text { if } \quad U_{a d, h}=U_{a d}^{h} .
$$

Proof. (i) Let us start with the case where $U_{a d, h}=U_{a d}^{h}$. For $0<h \leq h_{5}$, we have

$$
\begin{aligned}
& \frac{\delta}{2}\left\|\overline{\mathbf{u}}-\overline{\mathbf{u}}_{h}\right\|_{L^{2}\left(\omega ; \mathbb{R}^{m}\right)}^{2} \leq\left(J^{\prime}(\overline{\mathbf{u}})-J^{\prime}\left(\overline{\mathbf{u}}_{h}\right)\right)\left(\overline{\mathbf{u}}-\overline{\mathbf{u}}_{h}\right) \\
& =\left(J^{\prime}(\overline{\mathbf{u}})-J_{h}^{\prime}\left(\overline{\mathbf{u}}_{h}\right)\right)\left(\overline{\mathbf{u}}-\overline{\mathbf{u}}_{h}\right)+\left(J_{h}^{\prime}\left(\overline{\mathbf{u}}_{h}\right)-J^{\prime}\left(\overline{\mathbf{u}}_{h}\right)\right)\left(\overline{\mathbf{u}}-\overline{\mathbf{u}}_{h}\right) .
\end{aligned}
$$


With (4.12) in Lemma 4.13, we can estimate the last term as follows:

$$
\begin{aligned}
& \left(J_{h}^{\prime}\left(\overline{\mathbf{u}}_{h}\right)-J^{\prime}\left(\overline{\mathbf{u}}_{h}\right)\right)\left(\overline{\mathbf{u}}-\overline{\mathbf{u}}_{h}\right) \\
& =\int_{\omega}\left(\mathcal{C}^{*}\left(\overline{\mathbf{\Phi}}_{h}-\boldsymbol{\Phi}_{\overline{\mathbf{u}}_{h}}\right)+N\left(\overline{\mathbf{u}}_{h}-\overline{\mathbf{u}}_{h}\right)\right) \cdot\left(\overline{\mathbf{u}}-\overline{\mathbf{u}}_{h}\right) d x \\
& \leq C\left\|\overline{\mathbf{\Phi}}_{h}-\boldsymbol{\Phi}_{\overline{\mathbf{u}}_{h}}\right\|_{\mathbf{L}^{2}(\Omega)}\left\|\overline{\mathbf{u}}-\overline{\mathbf{u}}_{h}\right\|_{L^{2}\left(\omega ; \mathbb{R}^{m}\right)} \\
& \leq C h^{2}\left\|\overline{\mathbf{u}}-\overline{\mathbf{u}}_{h}\right\|_{L^{2}\left(\omega ; \mathbb{R}^{m}\right)} .
\end{aligned}
$$

Let us check what happens with the first term. From first order optimality conditions for problems $(\mathrm{P})$ and $\left(\mathrm{P}_{h}\right)$ we have

$$
\begin{aligned}
& J^{\prime}(\overline{\mathbf{u}})\left(\overline{\mathbf{u}}_{h}-\overline{\mathbf{u}}\right) \geq 0 \\
J_{h}^{\prime}\left(\overline{\mathbf{u}}_{h}\right)\left(\mathbf{u}_{h}^{*}-\overline{\mathbf{u}}_{h}\right)= & J_{h}^{\prime}\left(\overline{\mathbf{u}}_{h}\right)\left(\mathbf{u}_{h}^{*}-\overline{\mathbf{u}}\right)+J_{h}^{\prime}\left(\overline{\mathbf{u}}_{h}\right)\left(\overline{\mathbf{u}}-\overline{\mathbf{u}}_{h}\right) \geq 0 .
\end{aligned}
$$

Making the sum of these two expressions and using Lemma 4.17(2)-(3), we have

$$
\begin{aligned}
& J^{\prime}(\overline{\mathbf{u}})\left(\overline{\mathbf{u}}^{\prime}-\overline{\mathbf{u}}_{h}\right)-J_{h}^{\prime}\left(\overline{\mathbf{u}}_{h}\right)\left(\overline{\mathbf{u}}-\overline{\mathbf{u}}_{h}\right) \leq J_{h}^{\prime}\left(\overline{\mathbf{u}}_{h}\right)\left(\mathbf{u}_{h}^{*}-\overline{\mathbf{u}}\right) \\
& =J_{h}^{\prime}\left(\overline{\mathbf{u}}_{h}\right)\left(\mathbf{u}_{h}^{*}-\overline{\mathbf{u}}\right)-J^{\prime}(\overline{\mathbf{u}})\left(\mathbf{u}_{h}^{*}-\overline{\mathbf{u}}\right) \\
& =\int_{\omega}\left(\mathcal{C}\left(\overline{\mathbf{\Phi}}_{h}-\overline{\mathbf{\Phi}}\right)+N\left(\overline{\mathbf{u}}_{h}-\overline{\mathbf{u}}\right)\right) \cdot\left(\mathbf{u}_{h}^{*}-\overline{\mathbf{u}}\right) d x \\
& \leq C\left(\left\|\overline{\mathbf{\Phi}}_{h}-\overline{\mathbf{\Phi}}\right\|_{\mathbf{L}^{2}(\Omega)}+\left\|\overline{\mathbf{u}}_{h}-\overline{\mathbf{u}}\right\|_{L^{2}\left(\omega ; \mathbb{R}^{m}\right)}\right)\left\|\mathbf{u}_{h}^{*}-\overline{\mathbf{u}}\right\|_{L^{2}\left(\omega ; \mathbb{R}^{m}\right)} \\
& \leq C h\left(\left\|\overline{\boldsymbol{\Phi}}_{h}-\mathbf{\Phi}_{\overline{\mathbf{u}}_{h}}\right\|_{\mathbf{L}^{2}(\Omega)}+\left\|\boldsymbol{\Phi}_{\overline{\mathbf{u}}_{h}}-\overline{\mathbf{\Phi}}\right\|_{\mathbf{L}^{2}(\Omega)}+\left\|\overline{\mathbf{u}}_{h}-\overline{\mathbf{u}}\right\|_{L^{2}\left(\omega ; \mathbb{R}^{m}\right)}\right) \\
& \leq C h\left(h^{2}+\left\|\overline{\mathbf{u}}_{h}-\overline{\mathbf{u}}\right\|_{L^{2}\left(\omega ; \mathbb{R}^{m}\right)}\right) .
\end{aligned}
$$

From (4.19), (4.20), and (4.21), we deduce that therefore there exists a constant $C>0$, independent of $h$, such that

$$
\frac{\delta}{2}\left\|\overline{\mathbf{u}}-\overline{\mathbf{u}}_{h}\right\|_{L^{2}\left(\omega ; \mathbb{R}^{m}\right)}^{2} \leq C h^{3}+C h\left\|\overline{\mathbf{u}}_{h}-\overline{\mathbf{u}}\right\|_{L^{2}\left(\omega ; \mathbb{R}^{m}\right)} .
$$

We conclude with Young's inequality.

(ii) Now let us consider the case where $U_{a d, h}=U_{a d}$. We rewrite the previous steps by introducing the simplifications corresponding to this case. For $0<h \leq h_{5}$, we have

$$
\begin{aligned}
& \frac{\delta}{2}\left\|\overline{\mathbf{u}}-\overline{\mathbf{u}}_{h}\right\|_{L^{2}\left(\omega ; \mathbb{R}^{m}\right)}^{2} \leq\left(J^{\prime}(\overline{\mathbf{u}})-J^{\prime}\left(\overline{\mathbf{u}}_{h}\right)\right)\left(\overline{\mathbf{u}}-\overline{\mathbf{u}}_{h}\right) \\
& =\left(J^{\prime}(\overline{\mathbf{u}})-J_{h}^{\prime}\left(\overline{\mathbf{u}}_{h}\right)\right)\left(\overline{\mathbf{u}}-\overline{\mathbf{u}}_{h}\right)+\left(J_{h}^{\prime}\left(\overline{\mathbf{u}}_{h}\right)-J^{\prime}\left(\overline{\mathbf{u}}_{h}\right)\right)\left(\overline{\mathbf{u}}-\overline{\mathbf{u}}_{h}\right) .
\end{aligned}
$$

Since $U_{a d, h}=U_{a d}$, from the first order optimality conditions satisfied by $\overline{\mathbf{u}}$ and $\overline{\mathbf{u}}_{h}$ we have

$$
\left(J^{\prime}(\overline{\mathbf{u}})-J_{h}^{\prime}\left(\overline{\mathbf{u}}_{h}\right)\right)\left(\overline{\mathbf{u}}-\overline{\mathbf{u}}_{h}\right) \leq 0 .
$$

We have already seen that

$$
\left(J_{h}^{\prime}\left(\overline{\mathbf{u}}_{h}\right)-J^{\prime}\left(\overline{\mathbf{u}}_{h}\right)\right)\left(\overline{\mathbf{u}}-\overline{\mathbf{u}}_{h}\right) \leq C h^{2}\left\|\overline{\mathbf{u}}-\overline{\mathbf{u}}_{h}\right\|_{L^{2}\left(\omega ; \mathbb{R}^{m}\right)} .
$$

Therefore there exists a constant $C>0$ independent of $h$ such that

$$
\frac{\delta}{2}\left\|\overline{\mathbf{u}}-\overline{\mathbf{u}}_{h}\right\|_{L^{2}\left(\omega ; \mathbb{R}^{m}\right)}^{2} \leq C h^{2}\left\|\overline{\mathbf{u}}_{h}-\overline{\mathbf{u}}\right\|_{L^{2}\left(\omega ; \mathbb{R}^{m}\right)} .
$$


The proof is complete.

From the previous theorem and Lemmas 4.10 and 4.13 we deduce

$$
\begin{aligned}
& \left\|\overline{\mathbf{y}}-\overline{\mathbf{y}}_{h}\right\|_{\mathbf{H}_{0}^{1}(\Omega)}+\left\|\bar{p}-\bar{p}_{h}\right\|_{L_{0}^{2}(\Omega)} \leq C h, \\
& \left\|\overline{\mathbf{\Phi}}-\overline{\mathbf{\Phi}}_{h}\right\|_{\mathbf{H}_{0}^{1}(\Omega)}+\left\|\bar{\pi}-\bar{\pi}_{h}\right\|_{L_{0}^{2}(\Omega)} \leq C h .
\end{aligned}
$$

\section{REFERENCES}

[1] F. Abergel and E. Casas, Some optimal control problems of multistate equations appearing in fluid mechanics, RAIRO Modél. Math. Anal. Numér., 27 (1993), pp. 223-247.

[2] N. Arada, E. Casas, And F. Tröltzsch, Error estimates for the numerical approximation of a semilinear elliptic control problem, Comput. Optim. Appl., 23 (2002), pp. 201-229.

[3] E. CASAS, Optimality conditions for some control problems of turbulent flows, in Flow Control, IMA Vol. Math. Appl. 68, M. Gunzburger, ed., Springer-Verlag, New York, 1995, pp. 127147.

[4] E. CASAS, Error estimates for the numerical approximation of semilinear elliptic control problems with finitely many state constraints, ESAIM Control Optim. Calc. Var., 8 (2002), pp. 345-374.

[5] E. CASAS, Using piecewise linear functions in the numerical approximation of semilinear elliptic control problems, Adv. Comput. Math., 26 (2007), pp. 137-153.

[6] E. Casas, M. Mateos, And F. Tröltzsch, Error estimates for the numerical approximation of boundary semilinear elliptic control problems, Comput. Optim. Appl., 31 (2005), pp. 193219.

[7] E. CASAS AND J.-P. RAYMOND, Error estimates for the numerical approximation of Dirichlet boundary control for semilinear elliptic equations, SIAM J. Control Optim., 45 (2006), pp. $1586-1611$.

[8] L. Cattabriga, Su un problema al contorno relativo al sistema di equazioni di Stokes, Rend. Sem. Mat. Univ. Padova, 31 (1961), pp. 308-340.

[9] P. G. Ciarlet and J.-L. Lions, Eds., Handbook of Numerical Analysis, Vol. II, Handb. Numer. Anal. II, Finite Element Methods. Part I, North-Holland, Amsterdam, 1991.

[10] K. Deckelnick and M. Hinze, Semidiscretization and error estimates for distributed control of the instationary Navier-Stokes equations, Numer. Math., 97 (2004), pp. 297-320.

[11] K. ERIKSSON Improved accuracy by adapted mesh-refinements in the finite element method, Math. Comp. 44 (1985), pp. 321-343.

[12] P. Girault and P.-A. Raviart, Finite Element Methods for Navier-Stokes Equations. Theory and Algorithms, Springer-Verlag, Berlin, 1986.

[13] M. Gunzburger, L. Hou, and T. Svobodny, Analysis and finite element approximation of optimal control problems for the stationary Navier-Stokes equations with distributed and Neumann controls, Math. Comp., 57 (1991), pp. 123-151.

[14] M. Gunzburger, L. Hou, And T. Svobodny, Analysis and finite element approximation of optimal control problems for the stationary Navier-Stokes equations with Dirichlet controls, RAIRO Modél. Math. Anal. Numér., 25 (1991), pp. 711-748.

[15] M. D. Gunzburger, L. Hou, and T. P. Svobodny, Boundary velocity control of incompressible flow with an application to viscous drag reduction, SIAM J. Control Optim., 30 (1992), pp. 167-181.

[16] M. Hinze, A variational discretization concept in control constrained optimization: The linearquadratic case, Comput. Optim. Appl., 30 (2005), pp. 45-63.

[17] H.-C. LeE And S. Kim, Finite element approximation and computations of optimal Dirichlet boundary control problems for the Boussinesq equations, J. Korean Math. Soc., 41 (2004), pp. 681-715.

[18] J. C. De los Reyes, A primal-dual active set method for bilaterally control constrained optimal control of the Navier-Stokes equations, Numer. Funct. Anal. Optim., 25 (2004), pp. 657683.

[19] T. RoubiČEK And F. Tröltzsch, Lipschitz stability of optimal controls for the steady-state Navier-Stokes equations, Control Cybernet., 32 (2003), pp. 683-705.

[20] R. Temam, Navier-Stokes Equations, North-Holland, Amsterdam, 1979.

[21] F. Tröltzsch And D. Wachsmuth, Second-order sufficient optimality conditions for the optimal control of Navier-Stokes equations, ESAIM Control Optim. Calc. Var., 12 (2006), pp. 93-119. 